TO APPEAR IN THE ASTROPHYSICAL JOURNAL

Preprint typeset using LTEX style emulateapj v. 5/2/11

\title{
A LIGHT CURVE ANALYSIS OF CLASSICAL NOVAE: FREE-FREE EMISSION VS. PHOTOSPHERIC EMISSION
}

\author{
IZUMI HACHISU \\ Department of Earth Science and Astronomy, College of Arts and Sciences, The University of Tokyo, 3-8-1 Komaba, Meguro-ku, Tokyo 153-8902, Japan \\ AND \\ MARIKO KATO \\ Department of Astronomy, Keio University, Hiyoshi, Kouhoku-ku, Yokohama 223-8521, Japan \\ to appear in the Astrophysical Journal
}

\begin{abstract}
We analyzed light curves of seven relatively slower novae, PW Vul, V705 Cas, GQ Mus, RR Pic, V5558 Sgr, HR Del, and V723 Cas, based on an optically thick wind theory of nova outbursts. For fast novae, free-free emission dominates the spectrum in optical bands rather than photospheric emission and nova optical light curves follow the universal decline law. Faster novae blow stronger winds with larger mass loss rates. Since the brightness of free-free emission depends directly on the wind mass loss rate, faster novae show brighter optical maxima. In slower novae, however, we must take into account photospheric emission because of their lower wind mass loss rates. We calculated three model light curves of free-free emission, photospheric emission, and the sum of them for various WD masses with various chemical compositions of their envelopes, and fitted reasonably with observational data of optical, near-IR (NIR), and UV bands. From light curve fittings of the seven novae, we estimated their absolute magnitudes, distances, and WD masses. In PW Vul and V705 Cas, free-free emission still dominates the spectrum in the optical and NIR bands. In the very slow novae, RR Pic, V5558 Sgr, HR Del, and V723 Cas, photospheric emission dominates the spectrum rather than free-free emission, which makes a deviation from the universal decline law. We have confirmed that the absolute brightnesses of our model light curves are consistent with the distance moduli of four classical novae with known distances (GK Per, V603 Aql, RR Pic, and DQ Her). We also discussed the reason why the very slow novae are about $\sim 1$ mag brighter than the proposed maximum magnitude vs. rate of decline relation.

Subject headings: novae, cataclysmic variables — stars: individual (HR Del, PW Vul, V5558 Sgr, V705 Cas, V723 Cas)
\end{abstract}

\section{INTRODUCTION}

A classical nova is a thermonuclear runaway event on a mass-accreting white dwarf (WD) in a binary. When the mass of the hydrogen-rich envelope on the WD reaches a critical value, hydrogen ignites to trigger a nova outburst. Optical light curves of novae have a wide variety of timescales and shapes (e.g., Payne-Gaposchkin 1957; Duerbeck 1981; Strope et al. 2010; Hachisu \& Kato 2014). Hachisu \& Kato (2006) found that, in terms of free-free emission, optical and near-infrared (NIR) light curves of several novae follow a universal decline law. Their time-normalized light curves are almost independent of the WD mass, chemical composition of ejecta, and wavelength. Hachisu \& Kato (2006) also found that their UV $1455 \AA$ model light curves (Cassatella et al. 2002), interpreted as photospheric blackbody emission, are also time-normalized by the same factor as in the optical and NIR light curves. Using the fact that the time-scaling factor is closely related to the WD mass, the authors determined the WD mass and other parameters for a number of well-observed novae (e.g., Hachisu \& Kato 2007, 2010, 2014; Hachisu et al. 2008; Kato et al. 2009).

On the basis of the universal decline law, Hachisu \& Kato (2010) further obtained absolute magnitudes of their model light curves and derived their maximum magnitude vs. rate of decline (MMRD) relation. Such MMRD relations were empirically proposed, e.g., by Schmidt (1957),

hachisu@ea.c.u-tokyo.ac.jp marıko@educ.cc.ke1o.ac.jp della Valle \& Livio (1995), and Downes \& Duerbeck (2000). For individual novae, however, there is large scatter around the proposed trends (e.g., Downes \& Duerbeck 2000). Hachisu \& Kato's (2010) theoretical MMRD relation is governed by two parameters, one is the WD mass and the other is the initial envelope mass at the nova outburst, i.e., the ignition mass. The ignition mass depends on the mass-accretion rate to the WD. The higher the mass-accretion rate is, the smaller the ignition mass is (e.g., Nomoto 1982; Prialnik \& Kovetz 1995; Kato et al. 2014). In other words, the smaller the mass accretion rate is, the brighter the maximum magnitude is. So, they concluded that this second parameter (the ignition mass) explains scatter of the MMRD distribution of individual novae from the averaged trend that was determined mainly by the WD mass. Thus, the main trends of nova speed class were theoretically clarified.

In this way, the main properties of fast novae have been theoretically explained, in which free-free emission dominates the continuum spectra in optical and NIR bands. As far as free-free emission is the dominant source of nova optical light curves, there should be the universal decline law and we expect that novae follow Hachisu \& Kato's (2010) theoretical MMRD relation with the intrinsic scatter mentioned above. For slow novae, however, the universal decline law could not be applied because photospheric emission contributes substantially to the continuum spectra rather than free-free emission (Hachisu \& Kato 2014). Our aim of this paper is to analyze light curves of seven relatively slower novae, PW Vul, V705 Cas, GQ Mus, RR Pic, V5558 Sgr, 
HR Del, and V723 Cas, and to clarify how deviate their light curves from the universal decline law.

We organize the present paper as follows. Section 2 describes our strategy of light curve analysis. In Section 3, we start with a study of well-observed multi-wavelength lightcurves of the slow nova PW Vul and, through our method, we determine its relevant physical parameters such as the WD mass. Our method for nova light curves is also applied to the moderately fast nova V705 Cas in Section 4, to the fast nova GQ Mus in Section 5, and to the very slow novae, RR Pic, V5558 Sgr, HR Del, and V723 Cas in Section 6 Discussion and conclusions follow in Sections 7 and 8 Appendix $\mathrm{A}$ is devoted to a calibration of the absolute magnitude of our free-free model light curves and a theoretical MMRD relation. Appendix B]presents our time-stretching method for PW Vul.

\section{ANALYSIS ON VARIOUS LIGHT CURVE SHAPES OF CLASSICAL NOVAE}

Figure 1 shows optical light curves of our target novae, V723 Cas, HR Del, V5558 Sgr, RR Pic, GQ Mus, PW Vul, and V705 Cas on a linear timescale. These seven novae are plotted in the order of global decline rate. The light curves show a rich variety of shapes, so one might think that there are no common physical properties like the universal decline law. However, we can find common properties hidden in the complicated light curve shapes. For example, PW Vul shows an oscillatory behavior in the light curve but the overall decline trend and color evolution are very similar to other smoothlydeclining classical novae, as shown in Figure 2 (see Section 3 for details). Figure 2 depicts the time-normalized light curves of PW Vul and well-observed fast novae. Figures 3 and 4 also show the light curves and colors of V723 Cas, HR Del, V5558 Sgr, and RR Pic. Closely looking at the light curves in Figures 2, 3, and 4, we can see common properties as follows.

(1) There is a linear decline phase in the middle part of optical light curves in Figure 2. This phase is explained by free-free emission based on the optically thick winds (red solid line) and the light curves follow the universal decline law (Hachisu \& Kato 2006, 2010).

(2) In the linear decline phase, the colors are constant at $B-V=-0.03$ and $U-B=-0.97$, as indicated by a horizontal solid line in Figure 2(b) and 2(c), respectively. They are the colors of optically thick free-free emission spectra (Hachisu \& Kato 2014).

(3) There is another linear decline of a slope $t^{-3}$ in the very late phase in Figure 2(a). This phase corresponds to free expansion of a nebula with no additional wind mass loss (Hachisu \& Kato 2006).

(4) The UV $1455 \AA$ narrow band flux basically follows a time-normalized universal shape unless the flux is absorbed by dust. The theoretical flux (red solid line) represents a blackbody flux of the pseudophotosphere (Kato \& Hachisu 1994).

Novae blow optically thick winds, which are the origin of free-free emission. As far as the free-free emission dominates the continuum spectra, we can expect that novae follow the universal decline law. Even if there are wavy-structured or dust-blackout shapes, the overall light curves follow the universal decline law (e.g., Hachisu \& Kato 2006, 2007, 2010, 2014; Hachisu et al. 2008; Kato et al. 2009). On the other

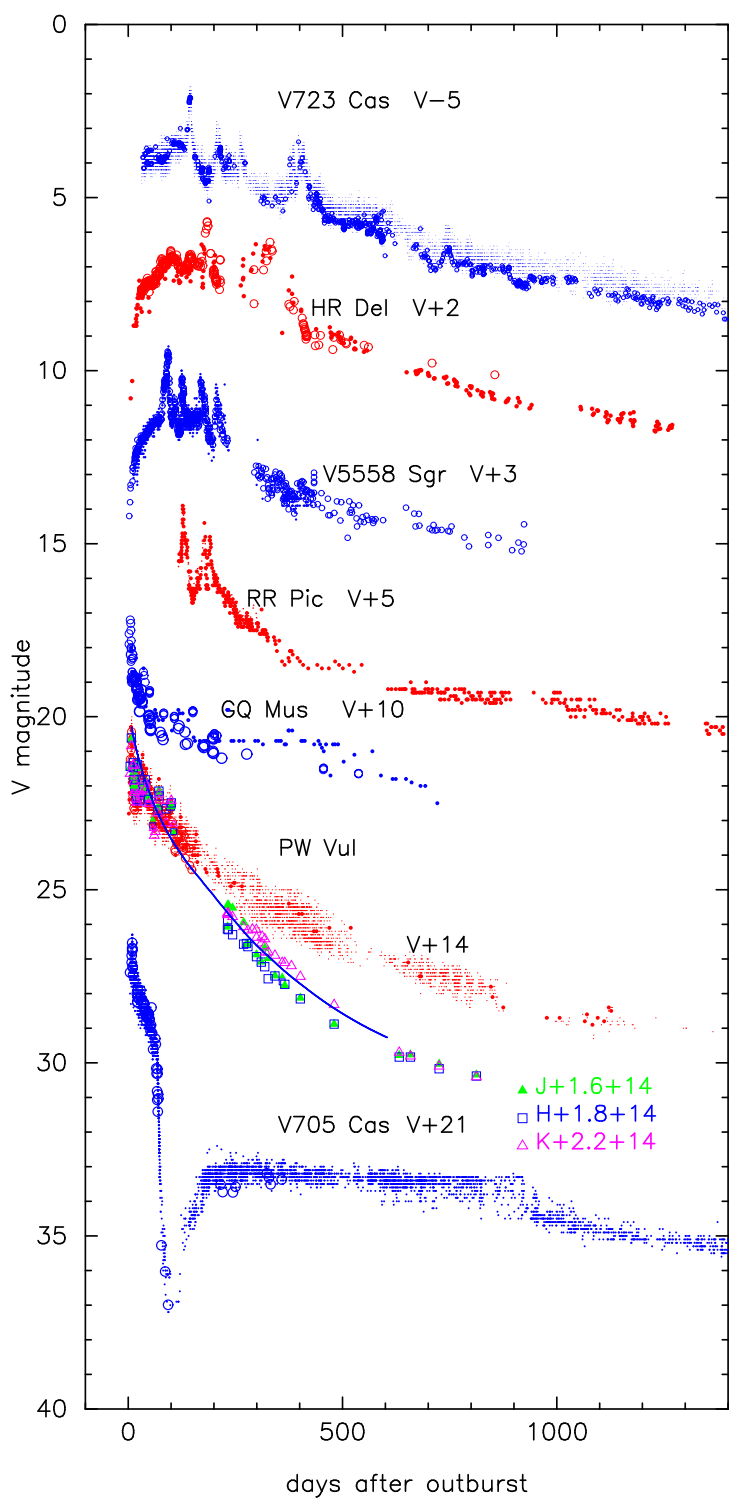

Figure 1. Visual and $V$ light curves for seven novae, from top to bottom, V723 Cas, HR Del, V5558 Sgr, RR Pic, GQ Mus, PW Vul, and V705 Cas in the order of global decline rates. Red or blue open circles denote $V$ magnitudes for each nova. Green filled triangles are $J$, blue open squares are $H$, and magenta open triangles are $K$ magnitudes of PW Vul. Their reference sources are found in the section of each object. Red or blue small dots represent visual magnitudes, all taken from the American Association of Variable Star Observers (AAVSO) archive except for GQ Mus. Blue small dots for GQ Mus are visual magnitude data collected by the Royal Astronomical Society of New Zealand. A blue solid line is our theoretical free-free emission light curve for a $0.83 M_{\odot}$ WD (see Section 3), which nicely fits with the near-infrared (NIR) light curves of PW Vul but not with the optical data that is contaminated by strong emission lines in the nebular phase.

hand, if photospheric emission dominates the nova continuum spectra, light curves do not follow the universal decline law.

Figure 5 shows a schematic illustration of nova continuum spectrum superposed on the various wavelength bands. Freefree spectra are plotted for a high wind mass loss rate (red solid line) and a low wind mass loss rate (blue solid line). In general, slower novae are related to less massive WDs which blow optically thick winds with relatively smaller wind mass loss rates (Kato \& Hachisu 1994). Thus, in such cases, we apply the universal decline law only to light curves in the NIR region but not to light curves in the optical regions. 

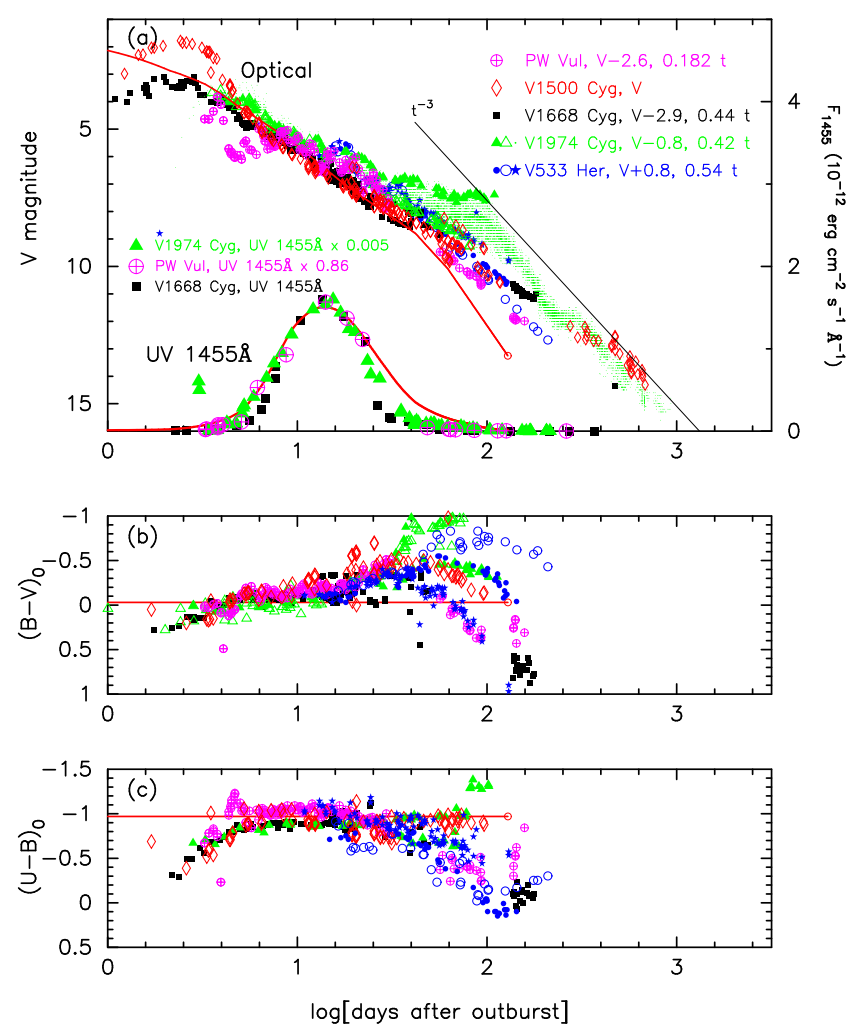

Figure 2. (a) $V$ band and UV $1455 \AA$ narrow band light curves, (b) $(B-V)_{0}$, and (c) $(U-B)_{0}$ color curves, for PW Vul (magenta open circles with plus sign inside), V1500 Cyg (red open diamonds), V1668 Cyg (black filled squares), and V1974 Cyg (green open and filled triangles). The reference sources are the same as those in Hachisu \& Kato (2006, 2010, 2014). Here, $(B-V)_{0}$ and $(U-B)_{0}$ denote dereddened colors. Each color is dereddened with $(B-V)_{0}=$ $B-V-E(B-V)$ and $(U-B)_{0}=U-B-0.64 E(B-V)$ (Rieke \& Lebofsky 1985). Those of V533 Her are also added: blue filled circles are taken from van Genderen (1963), blue open circles are from Chincarini (1964), and blue star symbols are from Shen et al. (1964). To make them overlap in the early decline phase, we shift horizontally their logarithmic times of PW Vul, V1668 Cyg, V1974 Cyg, and V533 Her by $-0.74=\log 0.182$, $-0.36=\log 0.44,-0.38=\log 0.42$, and $-0.28=\log 0.54$, and vertically their magnitudes by $-2.6,-2.9,-0.8$, and $+0.8 \mathrm{mag}$, respectively, as indicated in the figure. UV $1455 \AA$ fluxes of each nova are also rescaled against that of V1668 Cyg as indicated in the figure. Here, we assume the start of the day $(t=0)$ as JD 2445910.0 for PW Vul and JD 2438052.0 for V533 Her to correctly overlap them with other light curves. Red solid lines are our theoretical free-free emission and UV $1455 \AA$ light curves for a $0.83 M_{\odot}$ WD (see Section 3. We also draw two red horizontal lines of (b) $B-V=-0.03$ and (c) $U-B=-0.97$, both of which are the colors of optically thick free-free emission (see, e.g., Hachisu \& Kato 2014).

In this paper, we analyze the light curves of relatively slower novae in the following way.

(a) First, we determine the WD mass by applying properties (1)-(4) above.

(b) For this specified WD mass, we calculate the composite light curve of free-free plus photospheric emissions. From the fitting with the $V$ data, we determine the $V$ band distance modulus of $(m-M)_{V}$. We further estimate the distance to the nova if the color excess $E(B-V)$ is known.

(c) We compare our results with various properties in literature.

We first analyze PW Vul, V705 Cas, and GQ Mus based on the method mentioned above. They are the three novae in
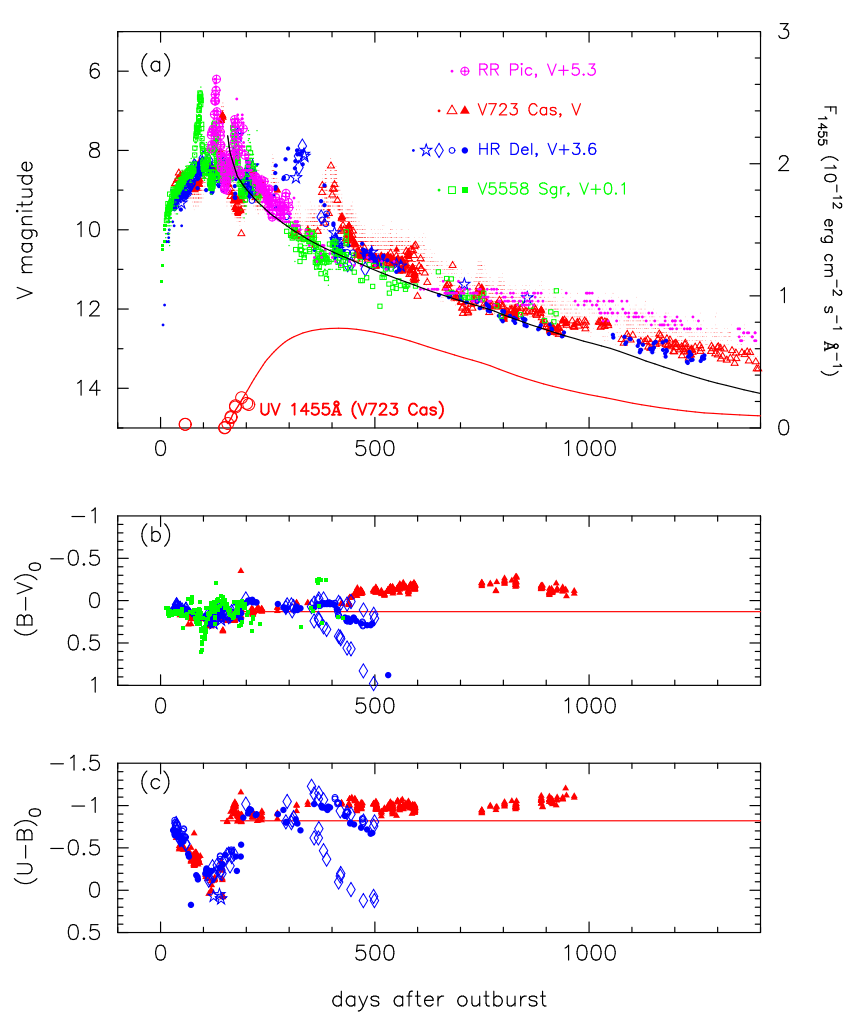

Figure 3. (a) Visual, $V$ band, and UV $1455 \AA$ narrow band light curves, (b) $(B-V)_{0}$, and (c) $(U-B)_{0}$ color curves, for RR Pic (magenta open circles with plus sign inside, magenta dots), V723 Cas (red open and filled triangles, red dots), HR Del (blue open stars, open circles, filled circles, blue dots), and V5558 Sgr (green open and filled squares, green dots). Here, $(B-V)_{0}$ and $(U-B)_{0}$ denote dereddened colors. To make them overlap in the early decline phase, we shift horizontally their times and vertically their magnitudes by $+5.3,0.0,+3.6$, and $+0.1 \mathrm{mag}$, respectively, as indicated in the figure. UV $1455 \AA$ fluxes of V723 Cas are plotted by large open circles. Black/Red solid lines are our theoretical $V /$ UV $1455 \AA$ light curves for a $0.51 M_{\odot}$ WD (see Section 6.4). We add two red horizontal lines of (b) $B-V=+0.13$ and (c) $U-B=-0.82$, both of which are the colors of optically thin free-free emission (see, e.g., Hachisu \& Kato 2014).

the lower part of Figure 1 Then, we go to the other four novae, RR Pic, V5558 Sgr, HR Del, and V723 Cas. These four novae show more or less similar light curves in their optical maximum and decline phases as shown in Figures 1, 3, and4

\section{PW VUL 1984\#1}

PW Vul (Nova Vulpeculae 1984\#1) was discovered by Wakuda on UT 1984 July 27.7 (Kosai et al. 1984) about a week before its optical maximum of $m_{V, \max }=6.3$ on UT 1984 August 4.1. The light curve is plotted in Figure 1 on a linear timescale and in Figure 2 on a logarithmic timescale. The Xray flux increased during the first year (Öegelman et al. 1987) and faded before the ROSAT observation $(1990-1999)$. No $\mathrm{X}$-ray data are available in the supersoft X-ray phase.

\subsection{Reddening and distance}

Andreae et al. (1991) obtained $E(B-V)=0.58 \pm 0.06$ from He II $\lambda 1640 / \lambda 4686$ ratio and $E(B-V)=0.55 \pm 0.1$ from the interstellar absorption feature at $2200 \AA$ for the reddening toward PW Vul. Saizar et al. (1991) reported $E(B-V)=0.60 \pm 0.06$ from He II $\lambda 1640 / \lambda 4686$ ratio. Duerbeck et al. (1984) estimated the extinction to be $E(B-$ $V)=0.45 \pm 0.1$ from galactic extinction in the direction toward the nova, whose galactic coordinates are $(l, b)=$ 
Table 1

Chemical composition of selected novae

\begin{tabular}{llllll}
\hline \hline \multicolumn{1}{c}{ object } & $\mathrm{H}$ & $\mathrm{CNO}$ & $\mathrm{Ne}$ & $\mathrm{Na}-\mathrm{Fe}$ & \multicolumn{1}{c}{ reference } \\
& & & & & \\
\hline HR Del 1967 & 0.45 & 0.074 & 0.0030 & $\ldots$ & Tylenda (1978) \\
DQ Her 1934 & 0.27 & 0.57 & $\ldots$ & $\ldots$ & Petitiean et al. (1990) \\
DQ Her 1934 & 0.34 & 0.56 & $\ldots$ & $\ldots$ & Williams et al. (1978) \\
V705 Cas 1993 \#2 & 0.57 & 0.25 & $\ldots$ & 0.0009 & Arkhipova et al. (2000) \\
V723 Cas 1995 & 0.52 & 0.064 & 0.052 & 0.042 & Iijima (2006) \\
GQ Mus 1983 & 0.37 & 0.24 & 0.0023 & 0.0039 & Morisset \& Péquignot (1996) \\
GQ Mus 1983 & 0.27 & 0.40 & 0.0034 & 0.023 & Hassall et al. (1990) \\
GQ Mus 1983 & 0.43 & 0.19 & $\ldots$ & $\ldots$ & Andreae \& Drechsel (1990) \\
RR Pic 1925 & 0.53 & 0.032 & 0.011 & $\ldots$ & Williams \& Gallagher (1979) \\
PW Vul 1984 \#1 & 0.69 & 0.066 & 0.00066 & $\ldots$ & Saizar et al. (1991) \\
PW Vul 1984 \#1 & 0.47 & 0.30 & 0.0040 & 0.0048 & Andreä et al. (1994) \\
PW Vul 1984 \#1 & 0.62 & 0.13 & 0.001 & 0.0027 & Schwarz et al. (1997) \\
PW Vul 1984 \#1 & 0.49 & 0.28 & 0.0019 & $\ldots$ & Andreae \& Drechsel (1990) \\
& & & & & \\
\hline
\end{tabular}
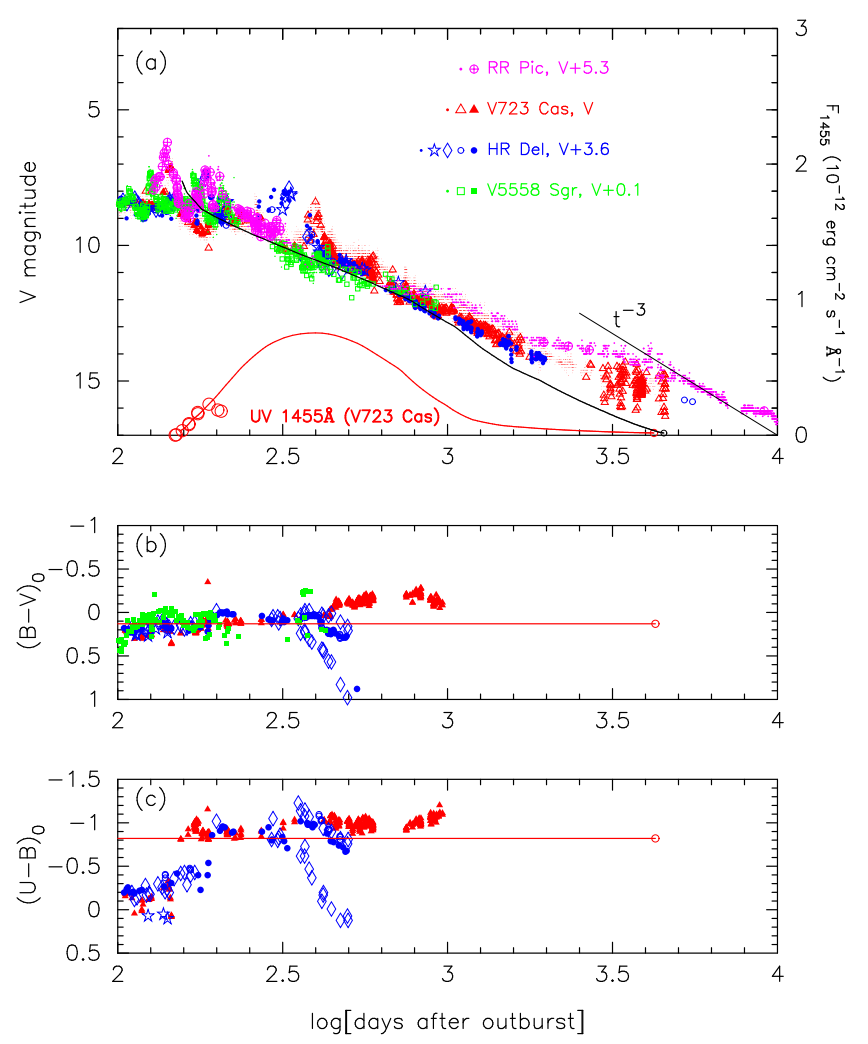

Figure 4. Same as Figure 3 but on a logarithmic timescale.

$(61.0983,+5.1967)$. For the galactic extinction, we examined the galactic dust absorption map in the NASA/IPAC Infrared Science Archive 1 , which is calculated on the basis of recent data from Schlafly \& Finkbeiner (2011). It gives $E(B-V)=0.43 \pm 0.02$ in the direction of PW Vul. The arithmetic mean of these four values is $E(B-V)=0.55 \pm 0.05$.

Recently, Hachisu \& Kato (2014) proposed a new method for determining reddening of classical novae. They identified a general course of $U B V$ color-color evolution and determined reddenings of novae by matching the track of a target nova with their general course. They obtained $E(B-V)=$ $0.55 \pm 0.05$ for PW Vul, which agrees well with the above mean value.

\footnotetext{
${ }^{1}$ http://irsa.ipac.caltech.edu/applications/DUST/
}

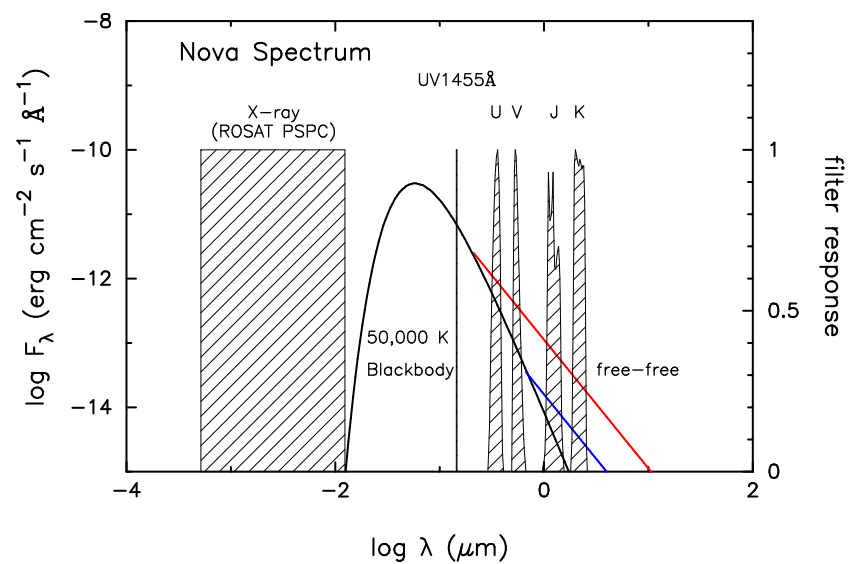

Figure 5. An illustrative example of spectral energy distribution of a classical nova with a photospheric temperature of $T_{\mathrm{ph}}=50,000 \mathrm{~K}$ as well as passbands of the photometric filters used in this work. The UV $1455 \AA$ (1445$1465 \AA)$ and supersoft X-ray $(0.1-2.4 \mathrm{keV})$ fluxes are calculated from blackbody spectrum while the $U, V, J$, and $K$ magnitudes are calculated from free-free emission spectrum. Note that the flux of free-free emission depends on the wind mass-loss rate, $\dot{M}_{\text {wind }}$. If the mass-loss rate is large, the flux of free-free emission dominates the spectrum in the optical and IR region as indicated by a red solid line. If it is small, the flux of free-free emission may dominate only in the IR region as indicated by a blue line. Supersoft X-ray flux is negligibly small for the adopted photospheric temperature.

As for the distance to PW Vul, a reliable estimate, $d=$ $1.8 \pm 0.05 \mathrm{kpc}$, was obtained by Downes \& Duerbeck (2000) through the nebular expansion parallax method. Adopting this value, together with $E(B-V)=0.55 \pm 0.05$, the distance modulus of PW Vul is

$$
\begin{aligned}
(m-M)_{V} & =5 \log \left(\frac{d}{10 \mathrm{pc}}\right)+3.1 E(B-V) \\
& =5 \log (180 \pm 5)+3.1(0.55 \pm 0.05) \\
& =13.0 \pm 0.2
\end{aligned}
$$

We also obtained $(m-M)_{V, \mathrm{PW} \text { Vul }}=13.0 \pm 0.1$ (see Appendix B) from "the time-stretching method" (Hachisu \& Kato 2010) of nova light curves. This value is consistent with Equation (1).

Figure 6 shows various distance-reddening relations for comparison. A horizontal magenta thick solid line with flanking thin lines represents the distance estimate of $d=1.8 \pm$ $0.05 \mathrm{kpc}$ (Downes \& Duerbeck 2000) mentioned above. A 


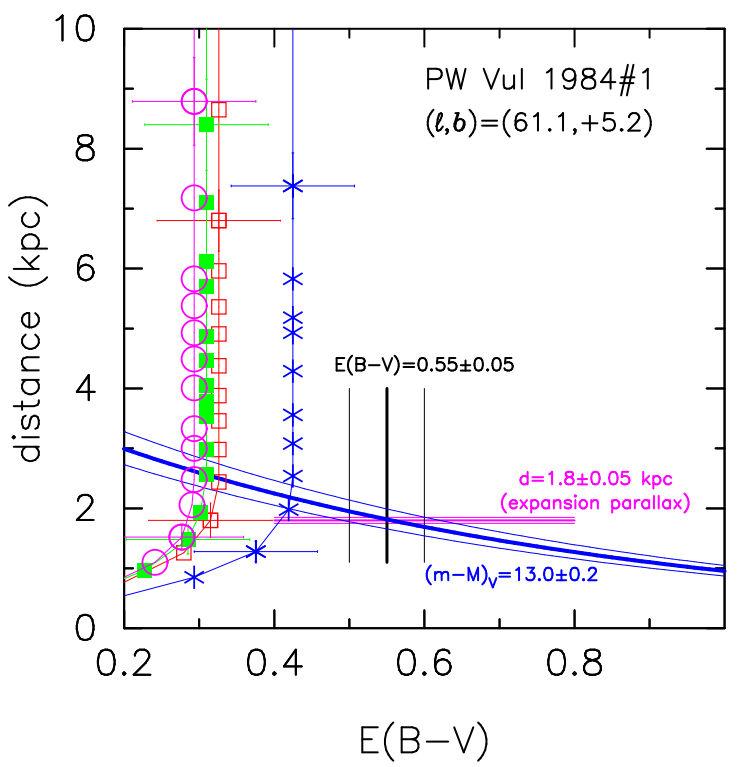

Figure 6. Distance-reddening relation toward PW Vul. Blue thick solid line with flanking thin solid lines denotes the distance-reddening relation calculated from the distance modulus of Equation (1), i.e., $(m-M)_{V}=$ $5 \log (d / 10 \mathrm{pc})+3.1 E(B-V)=13.0 \pm 0.2$. Vertical thick black solid line with flanking thin lines represents the color excess of $E(B-V)=0.55 \pm 0.05$. Horizontal magenta thick solid line with flanking thin lines represents the distance estimate of $d=1.8 \pm 0.05 \mathrm{kpc}$ from an expansion parallax method (Downes \& Duerbeck 2000). Distance-reddening relations in four directions close to PW Vul are shown by four different sets of data with error bars, taken from Marshall et al. (2006), i.e., $(l, b)=\left(61^{\circ} 00,5^{\circ} 00\right)($ red open squares), $\left(61.25,5^{\circ} .00\right)$ (green filled squares), $(61.00,5.25)$ (blue asterisks), and $(61.25,5.25)$ (magenta open circles). Typical error bars are shown only two points for each set to avoid complexity of lines.

vertical black solid line with flanking thin lines represents the color excess of $E(B-V)=0.55 \pm 0.05$. The reddeningdistance relation of Equation (11) is plotted by a blue thick solid line with flanking thin solid lines.

Marshall et al. (2006) published a three-dimensional dust extinction map of our galaxy in the direction of $-100.0 \leq$ $l \leq 100.0$ and $-10{ }^{\circ} 0 \leq b \leq+10.0$ with grids of $\Delta l=0.25$ and $\Delta b=0.25$, where $(l, b)$ are the galactic coordinates. Four sets of data with error bars in Figure 6 show distancereddening relations in four directions close to PW Vul: $(l, b)=$ $(61.00,5.00)$ (red open squares), $(61.25,5.00)$ (green filled squares), $(61.00,5.25)$ (blue asterisks), and $(61.25,5.25)$ (magenta open circles). The closest one is the blue asterisk relation that gives $E(B-V)=0.42 \pm 0.08$ at $d \approx 1.8 \pm 0.05 \mathrm{kpc}$. This value is consistent with $E(B-V)=0.43 \pm 0.02$ calculated from the NASA/IPAC dust map in the direction of PW Vul. Our value of $E(B-V)=0.55$ obtained above is larger than these values. However, the reddening trend of blue asterisks suggests a large deviation from the other three trends by $\Delta E(B-V) \approx 0.1$, i.e., reddening has patchy structure in this direction and further variation of $\Delta E(B-V) \sim 0.1$ is possible. Thus, we adopt $E(B-V)=0.55$ and $d \approx 1.8 \mathrm{kpc}$ in this paper.

\subsection{Chemical composition of ejecta}

One of the most intriguing properties of classical novae is the metal-enrichment of ejecta (e.g., Gehrz et al. 1998), which is ascribed to mixing with WD core material during outburst (e.g., Prialnik \& Kovetz 1995). PW Vul is not an exception of this general trend, as summarized in Table 1 There is a noticeable scatter in the abundance estimates, from $X=0.47$ to $X=0.69$. The arithmetic mean is $X=0.57$, $Y=0.22$, and $X_{\mathrm{CNO}}=0.19$ for $Z=0.02$. Here, $X, Y, Z$, and $X_{\mathrm{CNO}}$ are hydrogen, helium, heavy elements with solar abundance, and carbon-nitrogen-oxygen fractions in weight, respectively.

We took a simple parameterization for the degree of mixing between core material and accreted matter as $\eta_{\text {mix }}=(0.7 / X)-$ 1. Here we assume the solar composition for the accreted matter. Table 2 shows seven representative cases of degree of mixing, that is, $100 \%$ (denoted by "CO nova 1", "CO nova 2", and "Ne nova 1"), 55\% ("CO nova 3"), 25\% ("CO nova 4" and "Ne nova 2"), and $8 \%$ ("Ne nova 3"). We first adopt "CO nova 4," because it is closest to the above averaged values of PW Vul. Then, we discuss the dependence of light curves on the chemical composition.

\subsection{Contribution from photospheric emission}

Now we analyze the spectra of PW Vul assuming that the continuum flux $F_{\nu}$ is simply the sum of a blackbody spectrum of the temperature $T_{\mathrm{ph}}$ and an optically thick free-free emission with the electron temperature of $T_{\mathrm{e}}$, i.e.,

$$
F_{\nu}=f_{1} B_{\nu}\left(T_{\mathrm{ph}}\right)+f_{2} S_{\nu}\left(T_{\mathrm{e}}\right)
$$

where $\nu$ is the frequency, $B_{\nu}\left(T_{\mathrm{ph}}\right)$ is the Planckian of the photospheric temperature $T_{\mathrm{ph}}=T_{\mathrm{BB}}$, and $S_{\nu}\left(T_{\mathrm{e}}\right)$ is the freefree spectrum of the electron temperature $T_{\mathrm{e}}, f_{1}$ and $f_{2}$ are numeric constants (e.g., Nishimaki et al. 2008). Following Wright \& Barlow (1975), the free-free spectrum can be expressed as

$$
S_{\nu}\left(T_{\mathrm{e}}\right)=B_{\nu}\left(T_{\mathrm{e}}\right) K_{\nu}^{2 / 3}\left(T_{\mathrm{e}}\right),
$$

where the linear free-free absorption coefficient $K_{\nu}\left(T_{\mathrm{e}}\right)$ is given by

$$
K_{\nu}\left(T_{\mathrm{e}}\right)=3.7 \times 10^{8}\left[1-\exp \left(-\frac{h \nu}{k T_{\mathrm{e}}}\right)\right] Z^{2} g_{\nu}\left(T_{\mathrm{e}}\right) T_{\mathrm{e}}^{-1 / 2} \nu^{-3}
$$

in cgs units; $g_{\nu}\left(T_{\mathrm{e}}\right)$ is the Gaunt factor. In general, the Gaunt factor depends weakly on the frequency and temperature, but we assume it to be unity following Hachisu \& Kato (2014). So there are four fitting parameters, i.e., $f_{1}, f_{2}, T_{\mathrm{ph}}=T_{\mathrm{BB}}$, and $T_{\mathrm{e}}$. When $h \nu \ll k T_{e}$, Equation (3) can be expressed as (Wright \& Barlow 1975)

$$
S_{\nu}=23.2\left(\frac{\dot{M}_{\text {wind }}}{\mu v_{\infty}}\right)^{4 / 3} \frac{\nu^{2 / 3}}{D^{2}} \gamma^{2 / 3} g^{2 / 3} Z^{4 / 3} \mathrm{Jy},
$$

where $1 \mathrm{Jy}=10^{-26} \mathrm{~W} \mathrm{~m}^{-2} \mathrm{~Hz}^{-1}$; the ion number density is assumed equal to the total gas number density $n$ and the electron number density is equal to $\gamma$ times the ion number density; $\dot{M}_{\text {wind }}$ is the wind mass-loss rate in units of $M_{\odot} \mathrm{yr}^{-1} ; D$ is the distance in units of kpc; $v_{\infty}$ is the terminal wind velocity in units of $\mathrm{km} \mathrm{s}^{-1} ; \mu$ is the mean molecular weight; $Z$ is the charged-degree of ion (only in this formula); $\nu$ is the frequency in units of $\mathrm{Hz}$; and $g$ is the Gaunt factor. Equation (5) can be further simplified as

$$
S_{\nu} \propto \nu^{2 / 3}, \text { or } S_{\lambda} \propto \lambda^{-8 / 3},
$$

where $\lambda$ is the wavelength.

Figure 5 schematically shows the contributions of $B_{\lambda}$ and $S_{\lambda}$. When the wind mass loss rate is small, contribution of free-free emission is relatively small in Equation (2). Hachisu \& Kato (2014) decomposited the spectrum of PW Vul about 64 days after the outburst using Equation (2) and concluded that the free-free flux is comparable to the 
Table 2

Chemical composition of the present models

\begin{tabular}{lcclllll}
\hline \hline novae case & $X$ & $Y$ & $X_{\mathrm{CNO}}$ & $X_{\mathrm{Ne}}$ & $Z^{\mathrm{a}}$ & mixing $^{\mathrm{b}}$ & comments \\
\hline CO nova 1 & 0.35 & 0.13 & 0.50 & 0.0 & 0.02 & $100 \%$ & DQ Her \\
CO nova 2 $^{\mathrm{c}}$ & 0.35 & 0.33 & 0.30 & 0.0 & 0.02 & $100 \%$ & GQ Mus \\
CO nova 3 & 0.45 & 0.18 & 0.35 & 0.0 & 0.02 & $55 \%$ & V1668 Cyg \\
CO nova 4 & 0.55 & 0.23 & 0.20 & 0.0 & 0.02 & $25 \%$ & PW Vul \\
Ne nova 1 & 0.35 & 0.33 & 0.20 & 0.10 & 0.02 & $100 \%$ & V351 Pup \\
Ne nova 2 & 0.55 & 0.30 & 0.10 & 0.03 & 0.02 & $25 \%$ & V1500 Cyg \\
Ne nova 3 & 0.65 & 0.27 & 0.03 & 0.03 & 0.02 & $8 \%$ & QU Vul \\
Solar & 0.70 & 0.28 & 0.0 & 0.0 & 0.02 & $0 \%$ & \\
\hline
\end{tabular}

${ }^{a}$ Carbon, nitrogen, oxygen, and neon are also included in $Z=0.02$ with the same ratio as the solar composition Grevesse \& Anders 1989).

b Mixing between the helium layer + core material and the accreted matter with solar composition, which is calculated from $\eta_{\operatorname{mix}}=(0.7 / X)-1$.

c Free-free light curves for this chemical composition are tabulated in Table 2 of Hachisu \& Kato 2010).

d Free-free light curves for this chemical composition are tabulated in Table 3 of Hachisu \& Kato 2010).
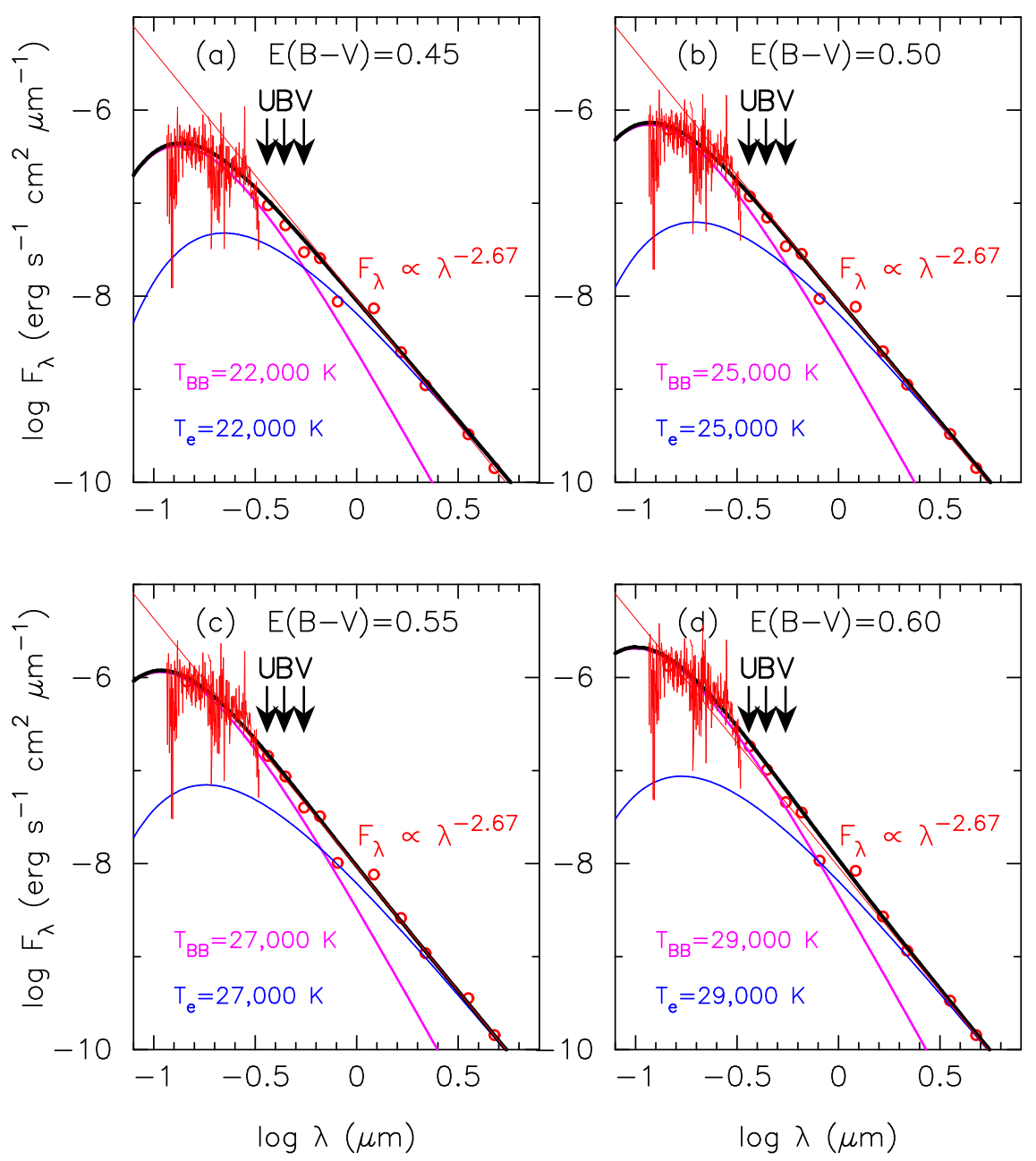

Figure 7. Dereddened spectrum of PW Vul, 64 days after the outburst (UT 1984 Sep $30=$ JD 2445973.5 ) for different extinctions: (a) $E(B-V)=0.45$, (b) $E(B-V)=0.50$, (c) $E(B-V)=0.55$, and (d) $E(B-V)=0.60$. Red solid line: IUE spectra, SWP24088 and LWP04458, are taken from the INES data archive server. Open red circles: $U B V R I$ data from Robb \& Scarfe (1995) and $J H K L M$ data from Gehrz et al. (1988). Global features of spectrum can be fitted with a combination (thick black solid line) of the blackbody with a temperature of (a) $T_{\mathrm{BB}}=22,000 \mathrm{~K}$, (b) $T_{\mathrm{BB}}=25,000 \mathrm{~K}$, (c) $T_{\mathrm{BB}}=27,000 \mathrm{~K}$, and (d) $T_{\mathrm{BB}}=29,000 \mathrm{~K}$ (thick magenta line) and the optically-thick, free-free emission with the same electron temperature of $T_{\mathrm{e}}$ (thick blue line). We also add a straight red thin solid line of $F_{\lambda} \propto \lambda^{-2.67}$, which corresponds to Equations (5) and [6), as the limiting case of $T_{\mathrm{e}}=\infty$ for optically-thick free-free emission. 
pseudo-photospheric flux in $V$ band. Here, we reanalyzed the same data and show them in Figure 7 assuming four different extinctions, i.e., (a) $E(B-V)=0.45$, (b) $E(B-V)=0.50$, (c) $E(B-V)=0.55$, and (d) $E(B-V)=0.60$. In our decomposition process, we simply assume that $T_{\mathrm{BB}}=T_{e}$ and change the temperature in steps of $1000 \mathrm{~K}$. We see that the blackbody emission gives a good approximation to the UV region and the free-free emission is a good fit to the IR region. In the region between them, we have a comparable contribution from the blackbody and free-free components.

These four decompositions of different sets of $T_{\mathrm{e}}=T_{\mathrm{BB}}$ and $E(B-V)$ more or less show similar good agreement. Among these four cases, $T_{\mathrm{BB}}=27,000 \mathrm{~K}$ in Figure 7 (c) is in best agreement with the temperature deduced from the light curve analysis in Section 3.4 It should be noted that the dereddened spectrum with $E(B-V)=0.55$ is closest to the straight line (red thin solid line) of $F_{\lambda} \propto \lambda^{-2.67}$ of Equation (6). Hauschildt et al. (1997) calculated synthetic NLTE nova spectra (see their Figure 10), in which the continuum flux has a slope of $F_{\lambda} \propto \lambda^{-2.7}$ in the range of $\lambda=0.2-2 \mu \mathrm{m}$ for $T_{\text {eff }}=25,000 \mathrm{~K}$ and $T_{\text {eff }}=30,000 \mathrm{~K}$. If we apply this slope directly to $\mathrm{PW}$ Vul, the spectrum is in best agreement with the reddening of $E(B-V)=0.55$ in Figure 7 (c).

The decomposition in Figure 7 c) indicates that the photospheric emission may substantially contribute in the optical light curve. In the next subsection, we calculate theoretical light curves, from the sum of free-free plus photospheric emission for the optical bands, and from only photospheric emission for the UV $1455 \AA$ band.

\subsection{Model Light Curves of "CO Nova 4"}

Now we make light curve models for PW Vul assuming the chemical composition of "CO Nova 4." We calculated nova light curves for various WD masses and fitted them to the observational data. The flux in the UV $1455 \AA$ band (a narrow band of 1445-1465 calculated as blackbody emission from the nova pseudophotosphere $\left(R_{\mathrm{ph}}\right.$ and $\left.T_{\mathrm{ph}}\right)$ using the optically-thick wind solutions in Kato \& Hachisu (1994). In our fitting process, we changed the WD mass from $0.80 M_{\odot}$ to $0.90 M_{\odot}$ in steps of $0.01 M_{\odot}$. In Figure 8, the $0.83 M_{\odot}$ WD model (black thin solid lines) shows reasonable agreement with the optical, NIR, and UV observations, in particular with the UV $1455 \AA$ observations. An arrow labeled "SED" indicates the date (Day 64) at which the spectrum in Figure 7 was secured. Our $0.83 M_{\odot}$ WD model has $T_{\mathrm{ph}}=28,000 \mathrm{~K}$ on this day, which is consistent with the blackbody temperature of $T_{\mathrm{BB}}=27,000 \mathrm{~K}$ determined in Section 3.3 .

From the UV $1455 \AA$ light curve fitting of the $0.83 M_{\odot}$ WD in Figure 8, we obtained the following distance-reddening relation, i.e.,

$$
\begin{aligned}
& 2.5 \log F_{\lambda 1455}^{\mathrm{mod}}-2.5 \log F_{\lambda 1455}^{\mathrm{obs}} \\
= & 2.5 \log \left(3.38 \times 10^{-12}\right)-2.5 \log \left((1.35 \pm 0.27) \times 10^{-12}\right) \\
= & 5 \log \left(\frac{d}{10 \mathrm{kpc}}\right)+8.3 \times E(B-V),
\end{aligned}
$$

where $F_{\lambda 1455}^{\text {mod }}=3.38 \times 10^{-12} \mathrm{erg} \mathrm{cm}^{-2} \mathrm{~s}^{-1} \AA^{-1}$ is the calculated UV $1455 \AA$ band flux at maximum of the $0.83 M_{\odot}$ WD model for an assumed distance of $10 \mathrm{kpc}$ and $F_{\lambda 1455}^{\mathrm{obs}}=$ $(1.35 \pm 0.27) \times 10^{-12} \mathrm{erg} \mathrm{cm}^{-2} \mathrm{~s}^{-1} \AA^{-1}$ is the maximum ob-

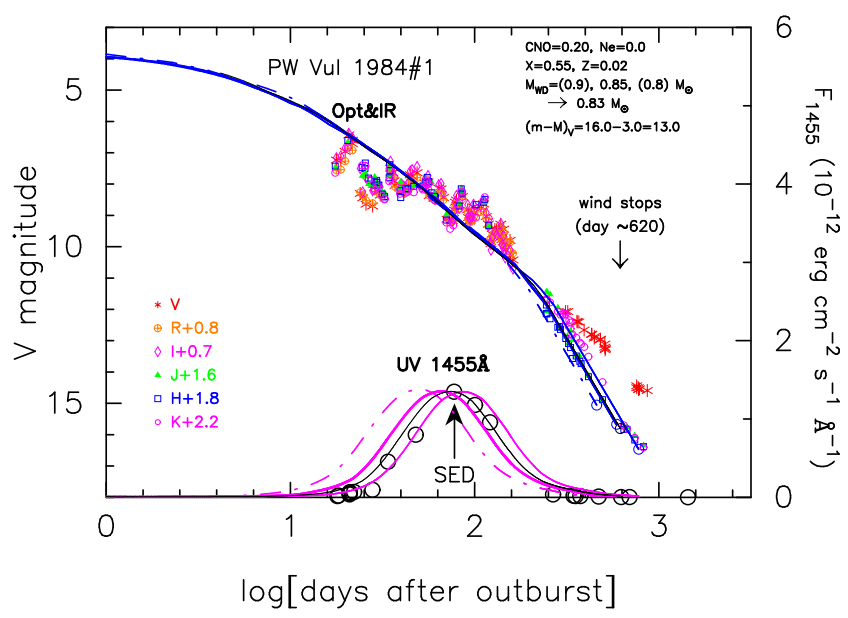

Figure 8. Optical, NIR, and UV $1455 \AA$ light curves of PW Vul. Here, we assume the start of the day $(t=0)$ as JD 2445897.0. Red asterisks represent $V$ magnitudes, which were taken from IAU Circular Nos. 3971 and 4091, and from Robb \& Scarfe (1995) and the AAVSO archive. Orange open circles with plus sign inside denote $R$ magnitudes shifted down by $0.8 \mathrm{mag}$, taken from Robb \& Scarfe (1995). Magenta open diamonds are I magnitudes shifted down by $0.7 \mathrm{mag}$, taken from Evans et al. (1990), Gehrz et al. (1988), Robb \& Scarfe (1995), and Williams et al. (1991). Green filled triangles are $J$ magnitudes shifted down by $1.6 \mathrm{mag}$, taken from Evans et al. (1990), Gehrz et al. (1988), and Williams et al. (1996). Blue open squares are $H$ magnitudes shifted down by $1.8 \mathrm{mag}$, taken from Evans et al. (1990), Gehrz et al. (1988), Robb \& Scarfe (1995), and Williams et al. (1996). Magenta open circles are $K$ magnitudes shifted down by 2.2 mag, taken from Evans et al. (1990), Gehrz et al. (1988), and Williams et al. (1996). Black large open circles are the UV $1455 \AA$ fluxes observed with $I U E$, taken from Cassatella et al. (2002). We plot three different WD mass models, $0.80 M_{\odot}$ (blue/magenta solid lines), $0.85 M_{\odot}$ (blue/magenta thick solid lines), and $0.90 M_{\odot}$ (blue/magenta dash-dotted lines), for the envelope chemical composition of "CO nova 4." We also added a fine mass model of $0.83 M_{\odot}$ (black thin solid lines). Optically thick winds stopped about 620 days after the outburst for the case of $0.83 M_{\odot}$ WD. The spectrum in Figure 7 was secured at the day denoted by an arrow labeled "SED."

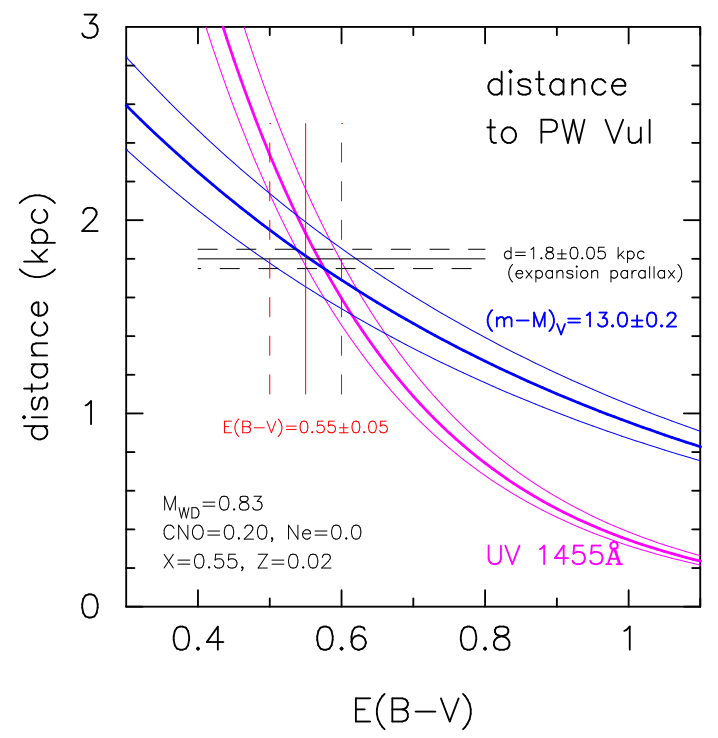

Figure 9. Same as Figure 6 but for the $0.83 M_{\odot}$ WD model with the chemical abundance of "CO nova 4." Blue solid line with flanking thin solid lines: distance-reddening relation calculated from Equation (1), labeled " $(m-M)_{V}=13.0 \pm 0.2$." Magenta solid line with flanking thin solid lines: distance-reddening relation calculated from Equation (7), i.e., the UV $1455 \AA$ flux fitting (labeled "UV $1455 \AA$ ") of the the $0.83 M_{\odot}$ WD model. 


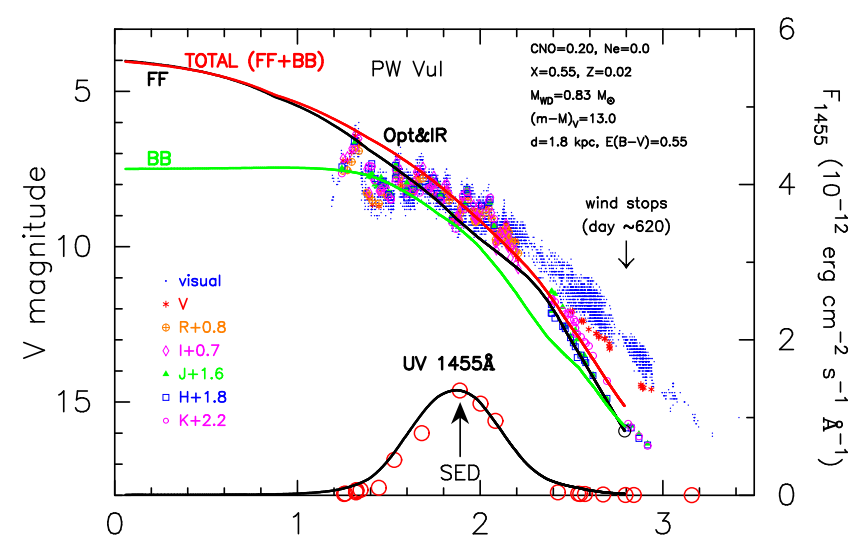

$\log t$ (day)

Figure 10. Same as Figure 8 but we added blackbody $V$ magnitude (green solid line labeled "BB") and total $V$ magnitude (red solid line labeled "TOTAL") for the distance modulus of $(m-M)_{V}=13.0$ in $V$ band. We determine the absolute magnitude of free-free emission light curve (black solid line labeled "FF") by $M_{\mathrm{w}}=3.0$, i.e., $m_{\mathrm{w}}=3.0+13.0=16.0$, at the end of wind phase (denoted by a black open circle) from fitting of our model light curve of total $V$ (red solid line) with the $V$ observation (red asterisks). We also added visual magnitudes (blue dots), which are taken from the AAVSO archive. Our model light curve reasonably fits with the early $V$ light curve but deviates from the visual observation in the later phase, that is, in the nebular phase. This deviation is due to strong emission lines such as [O III], which are not included in our model light curves. The spectrum in Figure 7 was secured at the day denoted by an arrow labeled "SED." Our $0.83 M_{\odot}$ WD model shows the photospheric temperature of about $T_{\mathrm{ph}}=28,000 \mathrm{~K}$ on this day, being consistent with the fitting temperature of $T_{\mathrm{BB}}=27,000 \mathrm{~K}$ in Figure 7 See text for more details.

served flux (Cassatella et al. 2002). Here we assume an absorption of $A_{\lambda}=8.3 \times E(B-V)$ at $\lambda=1455 \AA$ (Seaton 1979). The distance-reddening relation of Equation (7) is plotted by magenta solid lines in Figure 9 (labeled "UV 1455^”). Two relations of Equations (1) and (7) cross each other at the point of $(E(B-V), d)=(0.57 \pm 0.05 \mathrm{mag}, 1.75 \pm 0.3 \mathrm{kpc})$, being consistent with the observations summarized in Section 3.1 Therefore, we safely conclude that the WD mass of PW Vul is as massive as $\sim 0.83 M_{\odot}$ if the chemical composition is close to $X=0.55, Y=0.23, Z=0.02$, and $X_{\mathrm{CNO}}=0.20$.

Contrary to the UV $1455 \AA$ blackbody flux, our free-free model light curves are not yet calibrated. Optical light curves in Figure 8 are freely shifted in vertical direction to fit the observation because the proportionality constant in Equation (9) of Hachisu \& Kato (2006) is unknown for "CO nova 4." To fix the absolute magnitude of each light curve, we use the absolute magnitude of PW Vul as follows.

First, we calculate the light curve model of the $0.83 M_{\odot}$ WD and obtain the blackbody light curve in $V$ band (green solid line labeled "BB") as shown in Figure 10 Here, we adopt the distance modulus of $(m-M)_{V}=13.0$. Assuming a trial value for the proportionality constant $C$ in Equation (A1) of Appendix A.1, which is the same as Equation (9) of Hachisu \& Kato (2006), we obtain the absolute magnitude of the free-free model light curve (black solid line labeled "FF"). The total flux (red solid line labeled "TOTAL") is the sum of these two fluxes. However, in general, this total $V$ magnitude light curve does not fit well with the observed data. Then, we change the proportionality constant until the total $V$ flux fits well with the observed $V$ light curve. Figure 10 shows our final best fit model. We directly read $m_{\mathrm{w}}=16.0$ from Figure 10, where $m_{\mathrm{w}}$ is the apparent magnitude at the end of wind phase (open circle at the end of black solid line labeled
"FF"). Then, we obtain $M_{\mathrm{w}}=m_{\mathrm{w}}-(m-M)_{V}=16.0-13.0=$ 3.0 , where $M_{\mathrm{w}}$ is the absolute magnitude of free-free model light curve at the end of wind phase. Thus, the proportionality constant can be specified by $M_{\mathrm{w}}=3.0$ of the $0.83 M_{\odot}$ WD for PW Vul.

Based on the result of the $0.83 M_{\odot} \mathrm{WD}$ and applying the time-scaling law of free-free light curves to other WD mass models, we obtain the absolute magnitudes of free-free light curves for other WD masses with the chemical composition of "CO nova 4" (see Appendix A). The absolute magnitudes are specified by the value of $M_{\mathrm{w}}$ and listed in Table 4 for 0.55 $1.2 M_{\odot}$ WDs in steps of $0.05 M_{\odot}$.

It should be noted that our model light curve reasonably fits with the early $V$ light curve but deviates from the visual observation (small blue dots in Figure 10) in the nebular phase. On the other hand, our free-free model light curve almost perfectly fits with the NIR light curves even in the later phase. This deviation in visual magnitudes is owing to strong emission lines such as [O III], which are not included in our model (see Hachisu \& Kato 2006, for details). In the NIR region, free-free emission dominates the spectrum and our free-free light curve works well.

Thus, we may conclude that the effect of photospheric emission in $V$ band can be neglected in novae much faster than PW Vul because the mass-loss rate is large enough for freefree emission to dominate the spectrum in the optical and NIR region. We will discuss such examples of fast novae in Sections 7.1.1 and7.1.2. However, in less massive WDs or in novae much slower than PW Vul, we must take into account the contribution of photospheric emission. In this sense, PW Vul is a critical one between them.

\subsection{Effect of chemical composition}

The chemical composition of ejecta is usually not so accurately constrained as described in Section 3.2 and as tabulated in Table 1 If we adopt a chemical composition different from the true one, we could miss the WD mass and distance modulus of a nova. Therefore, we examine the dependence of our model light curve on the chemical composition, i.e., the degree of mixing. We adopted two other chemical compositions of "CO nova 2," a high degree of mixing, $\eta_{\text {mix }}=1.0(100 \%)$ and "Ne nova 2," a low degree of mixing, $\eta_{\text {mix }}=0.25(25 \%)$, mainly because their absolute magnitudes of free-free emission model light curves were already calibrated by Hachisu \& Kato (2010) independently of the light curve of PW Vul.

In a similar way to that in Section 3.4, we have obtained best fit models for the two chemical compositions mentioned above. Figure 11 shows our model light curves for (a) "CO Nova 2" and (b) "Ne Nova 2." In our fitting process, we changed the WD mass from $0.70 M_{\odot}$ to $0.90 M_{\odot}$ in steps of $0.01 M_{\odot}$. In Figure $11(\mathrm{a})$, the $0.78 M_{\odot}$ WD model shows reasonable agreement with the $V$ and UV observations. Also a good agreement is found for the $0.85 M_{\odot} \mathrm{WD}$ in Figure 11 b). It should be noted again that our model light curve fits well with the early $V$ light curve but deviates from the visual observation in the later phase, that is, in the nebular phase. This deviation is due to strong emission lines such as [O III], which are not included in our model light curves.

The flux in UV $1455 \AA$ band was calculated as blackbody emission from the nova pseudophotosphere using the optically-thick wind solutions in Kato \& Hachisu (1994) and Hachisu \& Kato (2006, 2010). From the UV $1455 \AA$ flux fit- 


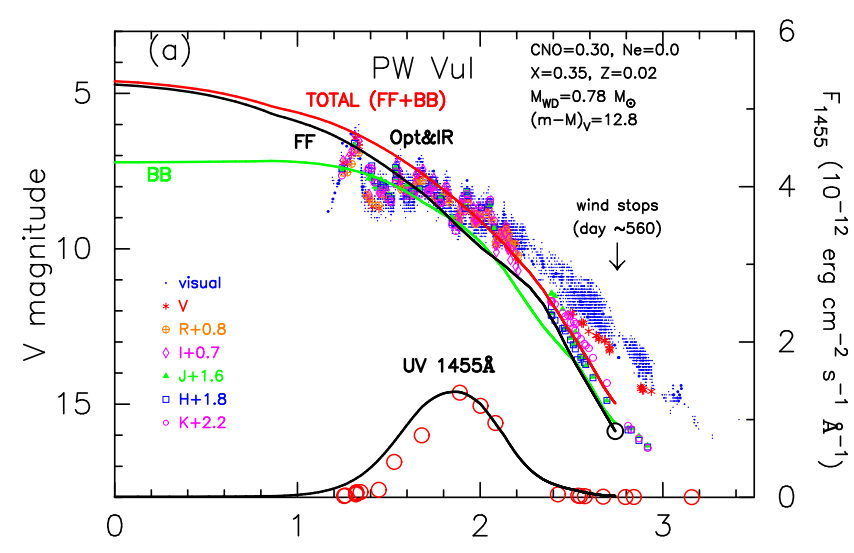

$\log t$ (day)

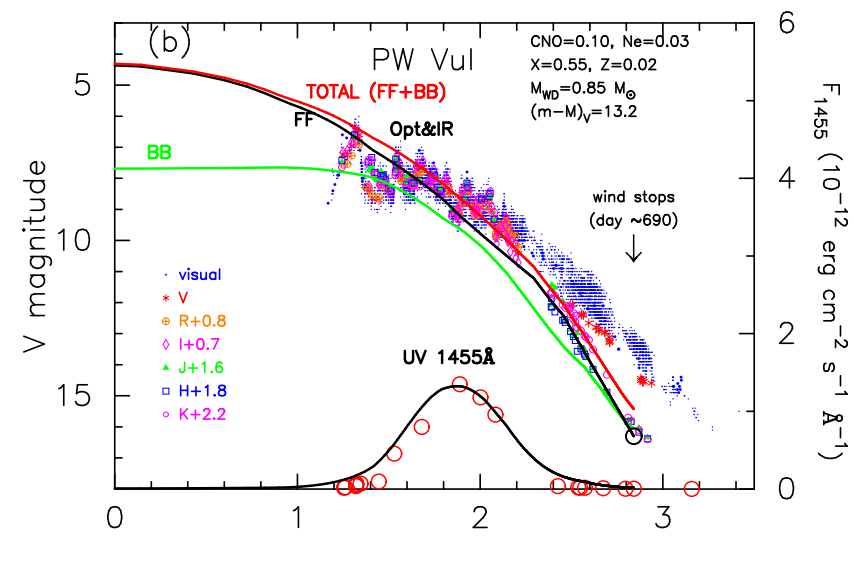

$\log t$ (day)

Figure 11. Same as Figure 10 but for (a) the $0.78 M_{\odot}$ WD model with the "CO nova 2" chemical composition and (b) the $0.85 M_{\odot}$ WD model with the "Ne nova 2" chemical composition. Optically thick winds stopped (a) about 560 days and (b) about 690 days after the outburst. We obtained (a) $(m-M)_{V}=12.8$ and (b) $(m-M)_{V}=13.2$ from the total $V$ flux fitting with the $V$ observation.

ting, we obtained the distance-reddening relation, which is

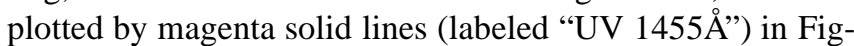
ure 12. Both the distance-reddening relations of UV $1455 \AA$ in Figure 12 are similar to that for "CO nova 4" in Figure 9

The total $V$ fluxes are calculated from the sum of free-free and blackbody fluxes. From the fitting, we also obtained the distance-reddening relation of (a) $(m-M)_{V}=12.8$ for the $0.78 M_{\odot}$ WD and (b) $(m-M)_{V}=13.2$ for the $0.85 M_{\odot}$ WD. The both are barely consistent with our adopted value of $(m-M)_{V}=13.0 \pm 0.2$ for PW Vul. The two distancereddening relations of total $V$ magnitude fitting are plotted by blue solid lines (labeled "TOTAL") in Figure 12 It is remarkable that our fitting misses the distance modulus only by 0.2 mag even if we assume a different chemical composition of $\Delta X=0.55-0.35=0.2$.

For a lower value of the hydrogen content $X$, the evolution timescale becomes shorter even if the WD mass is the same (see Kato \& Hachisu 1994; Hachisu \& Kato 2001). Therefore, a less massive WD of $0.78 M_{\odot}$ is fitted with the observation for a lower value of $X=0.35$ as shown in Figure 11 a). The wind mass loss rate is smaller for a less massive WD of $0.78 M_{\odot}$. The lower wind mass loss rate leads to fainter freefree emission and, as a result, a fainter total $V$ light curve. This is the reason for $(m-M)_{V}=12.8$, which is a bit smaller than the original value of $(m-M)_{V}=13.0$.
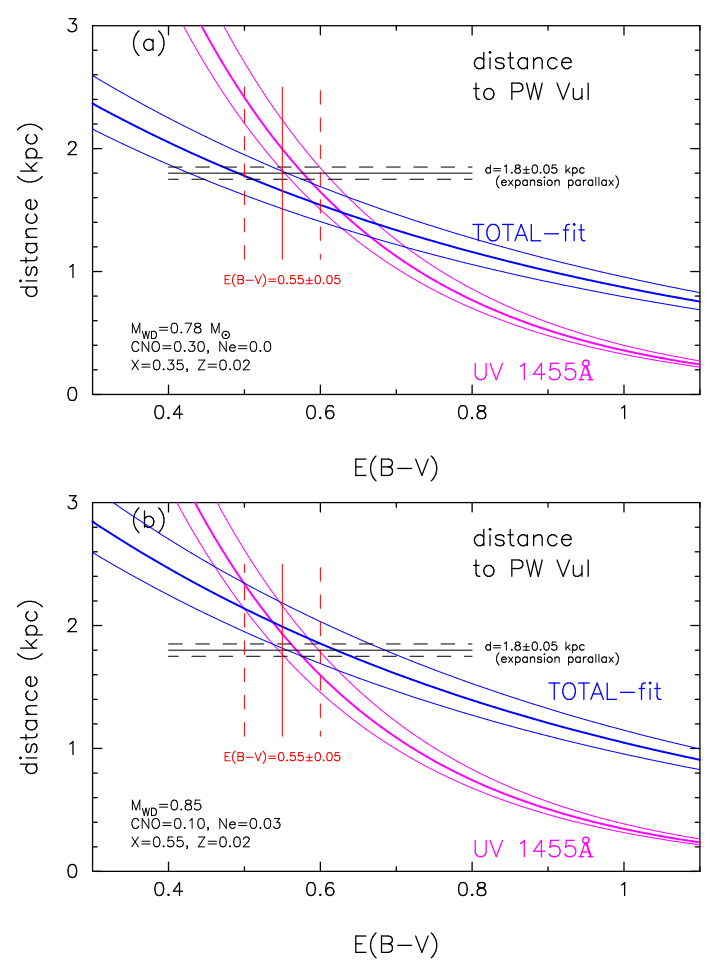

Figure 12. Distance-reddening relation toward PW Vul for the model of (a) $0.78 M_{\odot} \mathrm{WD}$ with the chemical composition of "CO nova 2" and (b) $0.85 M_{\odot}$ WD with the chemical composition of "Ne nova 2." Blue solid line with flanking thin solid lines: distance-reddening relation calculated from the fitting of total (free-free emission plus photospheric emission) $V$ model light curve (labeled "TOTAL-fit") obtained from Figure 11 Magenta solid line with flanking thin solid lines: distance-reddening relation calculated from the UV $1455 \AA$ flux fitting (labeled "UV $1455 \AA$ ") obtained from Figure 11. Two other constraints are also plotted; one is the distance of $d=1.8 \pm$ $0.05 \mathrm{kpc}$ determined by Downes \& Duerbeck (2000) with nebular expansion parallaxes and the other is the reddening of $E(B-V)=0.55 \pm 0.05$ determined from various constrains described in Section 3.1

For the chemical composition of "Ne nova 2," however, the hydrogen content of $X$ is the same as that of "CO nova 4." The difference is between $X_{\mathrm{CNO}}=0.20$ and $X_{\mathrm{CNO}}=0.10$. The CNO abundance is relevant to the nuclear burning rate and a lower value of $X_{\mathrm{CNO}}=0.10$ makes the evolution timescale longer. This requires a more massive WD of $0.85 M_{\odot}$ than the $0.83 M_{\odot}$ WD of $X_{\mathrm{CNO}}=0.20$ as shown in Figure 11 (b). The more massive WD blows stronger winds. This results in a brighter light curve of free-free emission and, as a result, a brighter total $V$ light curve. This is the reason for $(m-M)_{V}=$ 13.2, which is a bit larger than the original value of $(m-M)_{V}=$ 13.0.

In Figure 12 a), the two distance-reddening relations, i.e., "UV 1455^" and "TOTAL-fit," cross each other at the point of $(E(B-V), d)=(0.63 \mathrm{mag}, 1.5 \mathrm{kpc})$, being not consistent with $E(B-V)=0.55 \pm 0.05$ and $d=1.8 \pm 0.05 \mathrm{kpc}$. The degree of mixing may not be as high as $100 \%(X \approx 0.35)$ in PW Vul.

Figure 12 b) shows that the two relations cross at the point of $(E(B-V), d)=(0.54 \mathrm{mag}, 2.0 \mathrm{kpc})$. This value is consistent with the reddening estimate of $E(B-V)=0.55 \pm 0.05$ although the distance estimate is a bit larger than the distance estimate of $d=1.8 \pm 0.05 \mathrm{kpc}$. We may conclude that the lower degree of mixing ( $25 \%$ mixing) is more reasonable in PW Vul.

To summarize, we reached a reasonable distance-reddening result for a $25 \%$ mixing of "Ne Nova 2" but not for a $100 \%$ mixing of "CO Nova 2." Note that enrichment of neon 

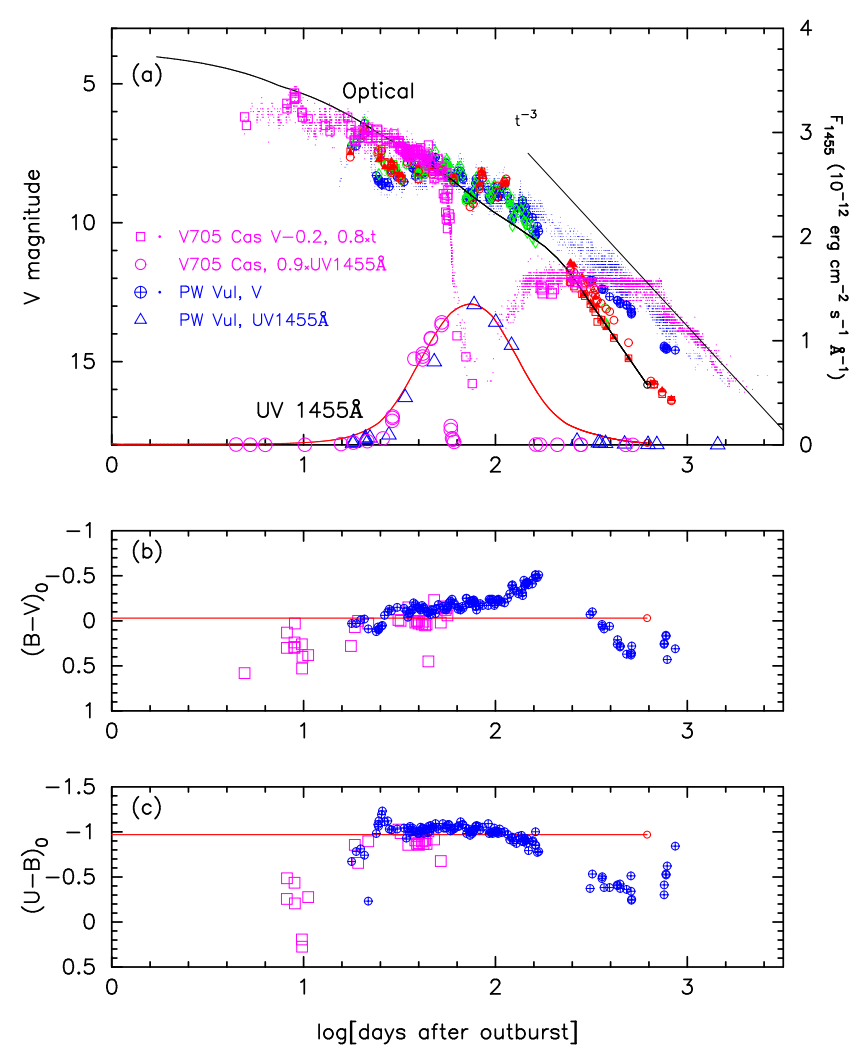

Figure 13. Same as Figure 2 but for V705 Cas (magenta open squares) and $\mathrm{PW}$ Vul (blue open circles with plus sign inside). The $U B V$ data of V705 Cas are taken from Munari et al. (1994b), Hric et al. (1998), and IAU Circular Nos. 5920 and 5929. Visual magnitudes (magenta/blue small dots) are taken from the AAVSO archive. We also added IJHK light curves of PW Vul (small green and red symbols), the data of which are the same as those in Figure 8 Panel (a) also shows the UV1455 ̊ light curves of V705 Cas (magenta open circles) and PW Vul (blue open triangles). In order to overlap the optical light curve of V705 Cas with that of PW Vul, we squeeze the light curve of V705 Cas by a factor of 0.8 in the direction of time and shifted the $V$ magnitudes of V705 Cas by -0.2 mag up as indicated in the figure. Model light curves of a $0.83 M_{\odot}$ WD are added: black solid line is the free-free $V$ and red solid line is the blackbody UV $1455 \AA$ (same as those in Figure 10 .

with hydrogen mass fraction being unchanged hardly influences the nova light curves because neon is not relevant either to nuclear burning (CNO-cycle) or to opacity (e.g., Kato \& Hachisu 1994; Hachisu \& Kato 2006, 2010). Therefore, the agreement in the lower $25 \%$ mixing model suggests that a lower degree of mixing $(\sim 25 \%)$ is reasonable rather than a higher degree of mixing $(\sim 100 \%)$. Unfortunately, there is significant scatter in the abundance determinations (see Table 1), but their averaged values of chemical composition, which show a $23 \%$ mixing, are close enough to those of "CO nova 4." Therefore, we may conclude that, through our method of model light curve fitting, one might discriminate between different degrees of mixing, at least, in terms of the hydrogen mass fraction $X$. We summarize our fitting result for PW Pul in Table 3.

\section{V705 CAS 1993}

Next, we analyze V705 Cas that shows a similar optical decline rate to PW Vul until a deep dust blackout started (see Figures 1 and 13). The chemical composition is also similar to PW Vul, as listed in Table 1 V705 Cas was discovered by Kanatsu on UT 1993 December 7 at about 6.5 mag (Nakano et al. 1993). It rose up to $m_{V}=5.5$ on UT December 17. Hric et al. (1998) estimated the decline rate of

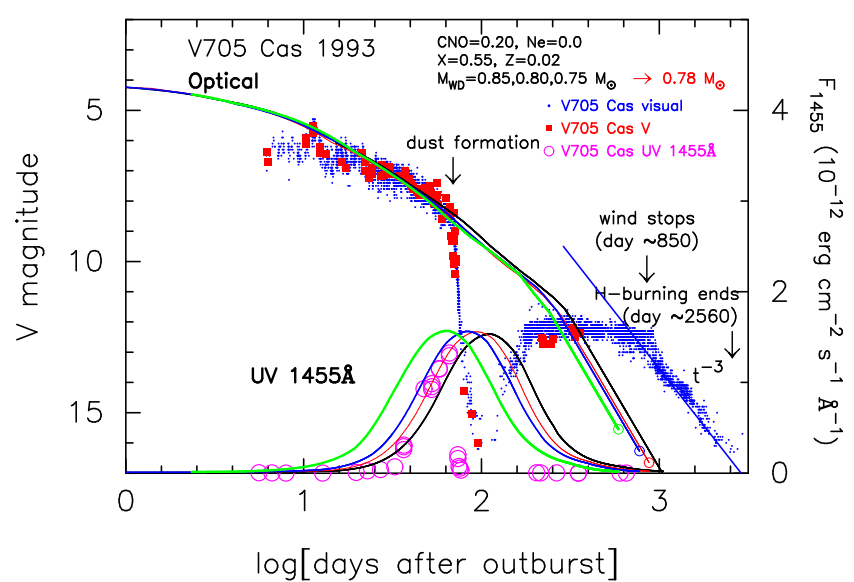

Figure 14. Optical and UV $1455 \AA$ A light curves of V705 Cas 1993. The observed $V$ magnitudes (filled red squares) are taken from Munari et al. (1994b), Hric et al. (1998), and IAU Circular Nos. 5920 and 5929. The visual magnitudes (small blue dots) are taken from the AAVSO archive. The UV 1455 ̊ flux (magenta open circles) are taken from Cassatella et al. (2002). We plot three different WD mass models: $0.75 M_{\odot}$ (black solid lines), $0.80 M_{\odot}$ (blue solid lines), and $0.85 M_{\odot}$ (green solid lines) for the envelope chemical composition of "CO nova 4 ." We add a $0.78 M_{\odot}$ WD model (red thin solid lines), which shows better fit to the UV $1455 \AA$ observation.

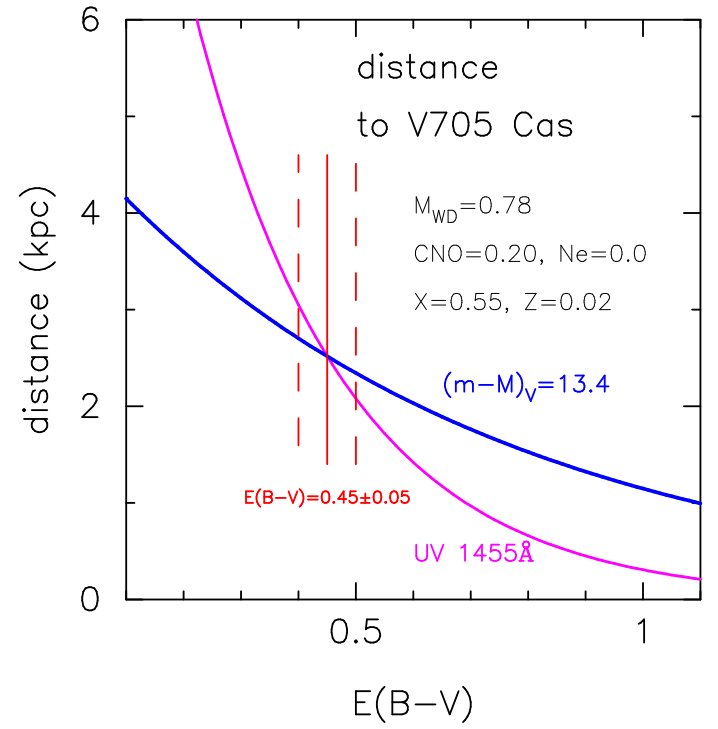

Figure 15. Distance-reddening relation for V705 Cas. We plot three relations, i.e., the color excess estimate of $E(B-V)=0.45 \pm 0.05$, distance modulus in $V$ band, and distance modulus in UV 1455 $\AA$ band, where we adopt the WD mass of $M_{\mathrm{WD}}=0.78 M_{\odot}$ with the elemental abundance of "CO nova 4 ." Three trends almost cross at the point of $E(B-V) \approx 0.45$ and $d \approx 2.5 \mathrm{kpc}$.

$t_{2, V}=33$ days, so V705 Cas is a moderately-fast nova.

Figure 13 compares the light curve and color evolutions of V705 Cas with those of PW Vul. Here, we squeeze the light curves of V705 Cas by a factor of 0.8. We see that these two novae show similar evolution. Using the time-stretching method, Hachisu \& Kato (2014) estimated the absolute magnitude of V705 Cas as $(m-M)_{V, V 705 \text { Cas }}=13.4$ (see Table 2 of Hachisu \& Kato 2014). We reanalyzed the data in Figure 13 in the same way as adopted for PW Vul in Appendix B, and obtained

$$
\begin{aligned}
(m-M)_{V, \mathrm{~V} 705 \mathrm{Cas}} & =(m-M)_{V, \mathrm{PW} \mathrm{Vul}}-\Delta V-2.5 \log 0.8 \\
& \approx 13.0+0.2+0.24 \approx 13.4
\end{aligned}
$$




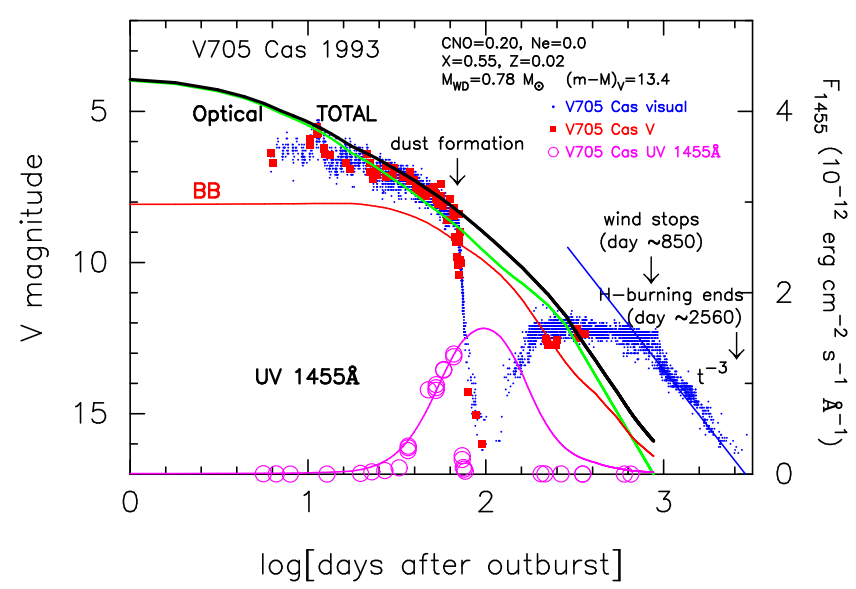

Figure 16. Same as Figure 14 but we added the blackbody flux in $V$ band (red solid line labeled "BB") and the total flux (thick black solid line labeled "TOTAL") of free-free (thick green solid line) plus blackbody in $V$ band for the $0.78 M_{\odot}$ WD model. Here, we assumed the distance modulus of ( $m-$ $M)_{V}=13.4$

$$
=5 \log \left(\frac{d}{10 \mathrm{pc}}\right)+3.1 \times E(B-V),
$$

where we use $(m-M)_{V, \mathrm{PW} \text { Vul }}=13.0$ determined in Section 3 . This value of $(m-M)_{V . V 705 \text { Cas }}=13.4$ is consistent with that obtained by Hachisu \& Kato (2014).

The distance modulus in UV $1455 \AA$ is estimated from our model light curve fitting. Figure 14 shows three model light curves of free-free emission for $M_{\mathrm{WD}}=0.75,0.80$, and $0.85 M_{\odot}$ WDs in steps of $0.05 M_{\odot}$ as well as the fine grid model of $0.78 M_{\odot}$ WD (red thin solid line) in steps of $0.01 M_{\odot}$. The distance-reddening relation of the $0.78 M_{\odot}$ WD model is derived from the UV $1455 \AA$ flux fitting, i.e.,

$$
\begin{aligned}
& 2.5 \log F_{\lambda 1455}^{\mathrm{mod}}-2.5 \log F_{\lambda 1455}^{\mathrm{obs}} \\
= & 2.5 \log \left(2.61 \times 10^{-12}\right)-2.5 \log \left(1.32 \times 10^{-12}\right) \\
= & 5 \log \left(\frac{d}{10 \mathrm{kpc}}\right)+8.3 \times E(B-V),
\end{aligned}
$$

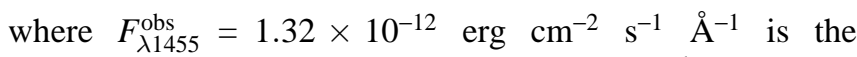
observed peak flux in Figure 14 and $F_{\lambda 1455}^{\bmod }=2.61 \times$ $10^{-12} \mathrm{erg} \mathrm{cm}^{-2} \mathrm{~s}^{-1} \AA^{-1}$ is the calculated flux of the $0.78 M_{\odot}$ model corresponding to the observed maximum at the distance of $10 \mathrm{kpc}$. Figure 15 shows these two distancereddening relations, i.e., Equation (8), labeled " $(m-M)_{V}=$ 13.4," and Equation (9), labeled "UV $1455 \AA$ A." These two lines cross at $E(B-V) \approx 0.45$ and $d \approx 2.5 \mathrm{kpc}$.

The reddening toward V705 Cas was estimated by Hric et al. (1998) to be $E(B-V)=0.38$ from the intercomparison of color indexes of the stars surrounding the nova selected from SAO catalog. They also obtained $E(B-V)=$ $(B-V)_{\mathrm{ss}}-(B-V)_{0, \mathrm{ss}}=0.32-(-0.11)=0.43$ from the intrinsic color at the stabilization stage (Miroshnichenko 1988). Hauschildt et al. (1995) obtained $E(B-V)=0.5$ from an assumption that the total (optical + UV) luminosity in an early phase is constant (see also Shore et al. 1994). A simple arithmetic mean of these values is $E(B-V)=0.44 \pm$ 0.05. The galactic dust absorption map of NASA/IPAC gives $E(B-V)=0.48 \pm 0.02$ in the direction toward V705 Cas, whose galactic coordinates are $(l, b)=(113 . \circ 595,-4.0959)$.
Hachisu \& Kato (2014) obtained $E(B-V)=0.45 \pm 0.05$ from the general course of novae in the color-color diagram. These values are all consistent with $E(B-V)=0.45 \pm 0.05$. Therefore, we use $E(B-V)=0.45 \pm 0.05$ in the present paper. Combining the distance modulus of $(m-M)_{V}=13.4$ in $V$ band and $E(B-V)=0.45$, we obtain the distance of $d=2.5 \mathrm{kpc}$. This reddening estimate is very consistent with our $E(B-V)=0.45$ as shown in Figure 15. This consistency strongly support the validity of our UV $1455 \AA$ light curve and time-stretching method of $V$ light curve.

Finally, we check the contribution of photospheric emission. Using this $0.78 M_{\odot}$ WD model, we calculated the brightness of photospheric emission in $V$ band (red solid line labeled "BB") and the total flux of free-free plus blackbody in $V$ band (thick black solid line labeled "TOTAL"), as shown in Figure 16. Here we use $M_{\mathrm{w}}=3.5$ for the $0.78 M_{\odot}$ WD from a linear interpolation between $M_{\mathrm{w}}=3.3\left(0.80 M_{\odot}\right)$ and $M_{\mathrm{w}}=3.8\left(0.75 M_{\odot}\right)$ in Table 4 For the distance modulus of $(m-M)_{V}=13.4$, the total $V$ light curve (thick black solid line) nicely fits with the $V$ observation. The contribution of photospheric emission is relatively smaller for V705 Cas. The obtained physical parameters are summarized in Table 3.

\section{GQ MUS 1983}

GQ Mus is a fast nova with $t_{2} \sim 18$ days (Whitelock et al. 1984). Its peak was missed, so we assume $m_{V, \max } \approx 7.2$ after Warner (1995). We plot the $V$, visual, $J, H, K$, UV $1455 \AA$, and X-ray light curves in Figure 17 . The $V$ data of GQ Mus are taken from Budding (1983) (red open triangles), Whitelock et al. (1984) (red open squares), and the Fine Error Sensor monitor on board IUE (red filled triangles) whereas the visual data are from the Royal Astronomical Society of New Zealand (small red open circles) and AAVSO (small red open circles) (see Hachisu et al. 2008, for more details). The $J$ (blue symbols), $H$ (orange symbols), $K$ (green symbols) light curves are taken from Whitelock et al. (1984) and Krautter et al. (1984). The UV $1455 \AA$ data are the same as those in Hachisu et al. (2008). The supersoft X-ray fluxes are taken from Shanley et al. (1995) and Orio et al. (2001). Krautter et al. (1984) suggested that the outburst took place 3-4 days before the discovery. In absence of precise estimates, we assumed that the outburst took place at $t_{\mathrm{OB}}=$ JD 2,445,348.0 (1983 January 13.5 UT), i.e., 4.6 days before the discovery by Liller on January 18.14, and adopted $t_{\mathrm{OB}}=\mathrm{JD} 2,445,348.0$ as day zero in the following analysis.

de Freitas Pacheco \& Codina (1985) determined the color excess of GQ Mus to be $E(B-V)=0.43$ and Péquignot et al. (1993) obtained $E(B-V)=0.50 \pm 0.05$ both from the hydrogen Balmer lines. Similar values were reported by Krautter et al. (1984) and Hassall et al. (1990), who found $E(B-V)=0.45$ and 0.50 , respectively, on the basis of the $2175 \AA$ feature in the early $I U E$ spectra. Hachisu et al. (2008) obtained $E(B-V)=0.55 \pm 0.05$ on the basis of the $2175 \AA$ feature and various line ratios. Hachisu \& Kato (2014) redetermined the color excess to be $E(B-V)=0.45 \pm 0.05$ by fitting the general tracks with that of GQ Mus in the $U B V$ color-color diagram. We adopt $E(B-V)=0.45 \pm 0.05$ in this paper, because the above estimates are all consistent with $E(B-V)=0.45 \pm 0.05$.

The chemical composition of GQ Mus were estimated by a few groups but scattered from $X=0.27$ to $X=0.43$ as listed in Table 1 So, we adopt two sets of chemical composition, i.e., "CO nova 2" and "CO nova 4." Figure 17 shows theoretical 

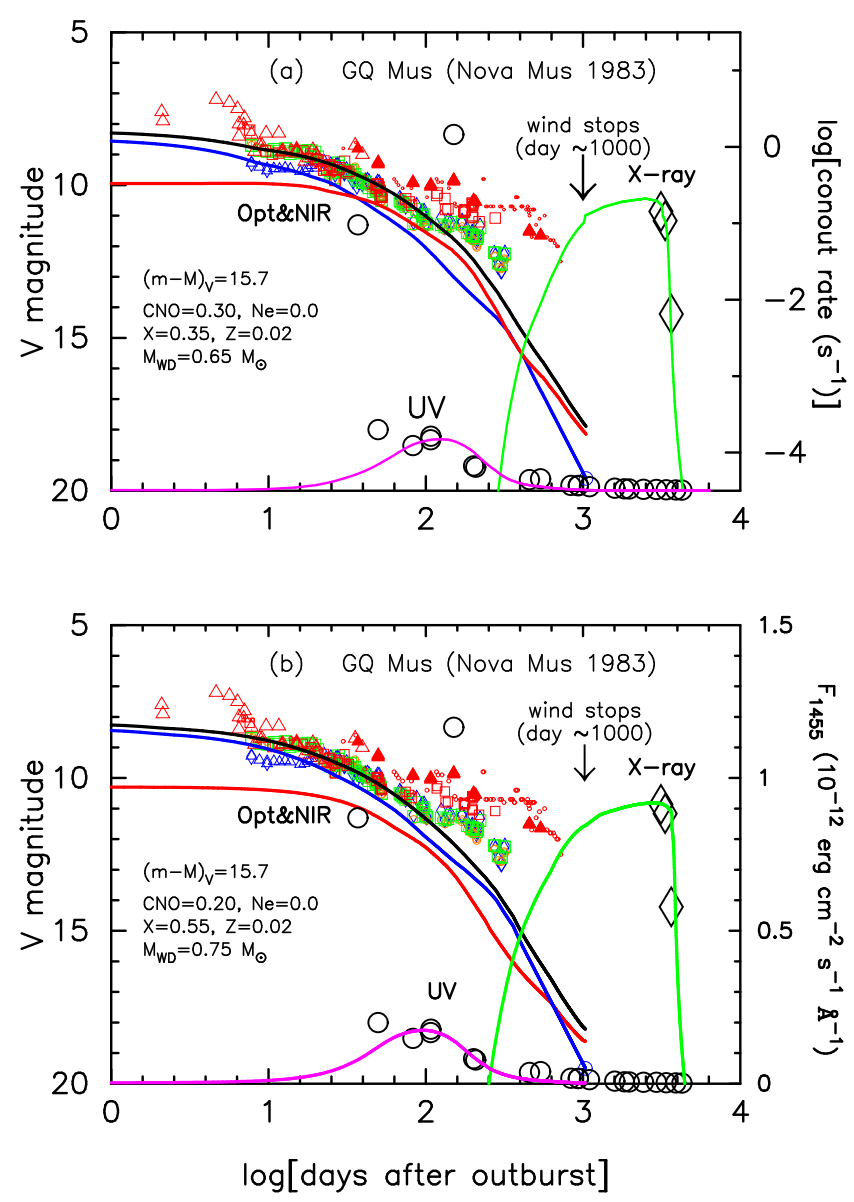

Figure 17. Multi-wavelength light curves for GQ Mus. The blackbody $V$ (red solid line), free-free (blue solid line), total $V$ magnitude (black solid line), UV $1455 \AA$ (magenta solid line), and X-ray $(0.1-2.4 \mathrm{keV}$, green solid line) light curves for (a) $0.65 M_{\odot}$ WD with the chemical composition of "CO Nova 2." and (b) $0.75 M_{\odot}$ WD with the chemical composition of "CO Nova 4." The distance modulus of $(m-M)_{V}=15.7$ is obtained for the both models, (a) and (b), from light curve fitting of the total $V$ flux (photospheric emission plus free-free emission). The observational data are the same as those cited in Hachisu et al. (2008). We shifted the observed $J$ (blue symbols), $H$ (orange symbols), and $K$ (green symbols) magnitudes down by 3.0, 2.4, and 3.0 mag, respectively.

light curves for the chemical composition of (a) "CO nova 2" and (b) "CO nova 4." These light curves are the best fit ones obtained by Hachisu et al. (2008) based on the free-free emission, UV $1455 \AA$, and supersoft X-ray model light curves. We calculated the photospheric emission and total emission model $V$ light curves and added them to the figure.

We calculated the total $V$ magnitudes for the $0.65 M_{\odot}$ WD with "CO nova 2." Here we used the absolute magnitudes of free-free emission model light curves in Table 2 of Hachisu \& Kato (2010). Figure 17 a) shows that the photospheric emission significantly contributes to the total flux in $V$ band and its effect improved the fitting. We obtain $(m-M)_{V}=15.7$ from fitting, i.e.,

$$
\begin{aligned}
(m-M)_{V} & =15.7 \\
& =5 \log \left(\frac{d}{10 \mathrm{pc}}\right)+3.1 \times E(B-V) .
\end{aligned}
$$

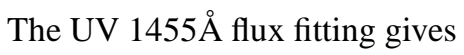

$$
2.5 \log F_{\lambda 1455}^{\mathrm{mod}}-2.5 \log F_{\lambda 1455}^{\mathrm{obs}}
$$

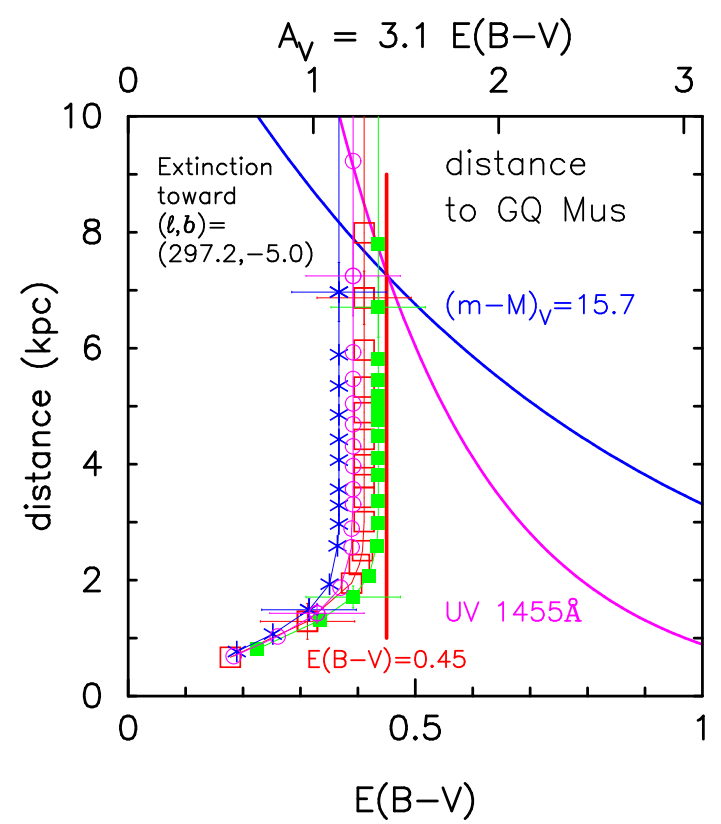

Figure 18. Distance-reddening relations toward GQ Mus. A blue thick solid line denotes Equation [10), i.e., $(m-M)_{V}=15.7$. A magenta thick solid line represents Equation (11, i.e., the UV $1455 \AA$ fit. Vertical red thick solid line indicates the color excess of $E(B-V)=0.45$. Four sets of data with error bars show distance-reddening relations in four directions close to GQ Mus of $(l, b)=(297.2118,-4.9959):(l, b)=(297.00,-4.75)$ (red open squares), $(297.25,-4.95)$ (green filled squares), $\left(297 . \circ 00,-5 .^{\circ} 00\right)$ (blue asterisks), and $(297.25,-5 \circ 00)$ (magenta open circles), taken from Marshall et al. (2006).

$$
\begin{aligned}
& =2.5 \log \left(2.97 \times 10^{-12}\right)-2.5 \log \left(1.78 \times 10^{-13}\right) \\
& =5 \log \left(\frac{d}{10 \mathrm{kpc}}\right)+8.3 \times E(B-V),
\end{aligned}
$$

where $F_{\lambda 1455}^{\text {mod }}=2.97 \times 10^{-12} \mathrm{erg} \mathrm{cm}^{-2} \mathrm{~s}^{-1} \AA^{-1}$ is the calculated peak flux of the $0.65 M_{\odot}$ model at the distance of $10 \mathrm{kpc}$ corresponding to the magenta solid line in Figure 17 (a) and

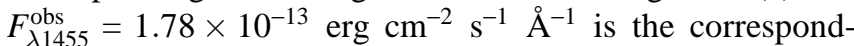
ing observed flux at the same epoch. We plot these two distance-reddening relations of Equations (10) and (11) in Figure 18 together with Marshal et al.'s (2006) relation and $E(B-V)=0.45$ toward GQ Mus. All trends cross consistently at $d \approx 7.3 \mathrm{kpc}$ and $E(B-V) \approx 0.45$. The galactic dust absorption map of NASA/IPAC gives $E(B-V)=0.42 \pm 0.01$ in the direction toward GQ Mus, whose galactic coordinates are $(l, b)=(297.2118,-4.9959)$, being consistent with our obtained value of $E(B-V)=0.45 \pm 0.05$.

For the $0.75 M_{\odot}$ WD of "CO Nova 4" in Figure 17(b), we also obtain $(m-M)_{V}=15.7$ for the total $V$ light curve fitting. The UV $1455 \AA$ fitting also shows a similar relation as Equation (11). Therefore, the distance-reddening relations are almost the same as those for "CO Nova 2." These fitting results are summarized in Table 3 .

Hachisu et al. (2008) obtained $(m-M)_{V}=14.7$ mainly from various MMRD relations. This old value is $1.0 \mathrm{mag}$ smaller than our new value, suggesting that the MMRD relations are not reliable for individual novae (see Section 7.2 and Figure 31 for the MMRD values of GQ Mus).

Figure 17 shows two UV flashes around Day 37 and Day 151 , the latter of which was a secondary outburst noticed by Hassall et al. (1990). The secondary outburst around Day 151 had actually the appearance of a "UV flash" because of its especially large amplitude at short wavelengths. Indeed, 
compared with the IUE low resolution observations obtained just before and after this event (Days 108 and 202), the UV flux increased by a factor of 9 at $1455 \AA$ and by a factor 2.2 at $2885 \AA$, while the visual flux increased only by a factor of 1.5. Hachisu et al. (2008) discussed this UV flash in more detail. Therefore, we excluded these points on Days 37, 49, and 151 from our UV light curve fittings because our model light curves follow only gradual increase and decrease in the UV flux.

As discussed in Sections 3 and $4, V$ and visual magnitudes are contaminated by strong emission lines, causing an upward deviation from our free-free models. In GQ Mus, forbidden [O III] $\lambda \lambda 4959,5007$ emission lines already appeared on Day 39 (Krautter et al. 1984). At about this date the observed visual light curve did actually start to show an upward deviation from the total flux model. On the other hand, $J H K$ bands are not so heavily contaminated by emission lines, as shown in Figure 17

GQ Mus is considered to be a super-bright nova. The observed $V$ magnitudes in Figure 17 shows about $1.5 \mathrm{mag}$ brighter than our model light curve in the earliest phase (until Day 8). A similar excess is present in the super-bright nova V1500 Cyg as shown in Figure 2 a) (see also della Valle 1991; Hachisu \& Kato 2006). We regard the early excess in $V$ magnitude (< Day 8 ) of GQ Mus as the superbright phase and exclude this phase from fitting, because the spectra in these super-bright phase are similar to blackbody rather than free-free emission (e.g., Gallagher \& Ney 1976, for V1500 Cyg). V1500 Cyg is a polar system (see, e.g., Schmidt et al. 1987; Schmidt \& Stockman 1987). GQ Mus is also suggested to be a polar system (Diaz \& Steiner 1989, 1994). This suggests a possibility that some of polar systems become a super-bright nova.

\section{VERY SLOW NOVAE, RR PIC, V5558 SGR, HR DEL, AND V723 CAS}

In this section we analyze the very slow novae, RR Pic, V5558 Sgr, HR Del, and V723 Cas. These novae have similar complex light curves of multiple peak as shown in Figures 1 and 3 . Here, we adopt Kato \& Hachisu's (2009, 2011) explanation for the multiple peak. They showed that there are two kinds of envelope solutions for the same envelope mass and WD mass, one is a static and the other is a wind mass loss solution, in a narrow range of WD mass, $0.5 M_{\odot} \lesssim M_{\mathrm{WD}} \lesssim 0.7 M_{\odot}$. On these WDs, nova outbursts begin quasi-statically and then undergo a transition from static to wind evolution. During the transition, the nova accompanies oscillatory activity and begins to blow massive winds after the transition is completed. Thus, we apply our method of optically thick wind solutions to the light curves only after the transition is completed.

The light curves of these four novae are very similar to each other and their chemical compositions were obtained to be $X=0.53$ for RR Pic, $X=0.45$ for HR Del, and $X=0.52$ for V723 Cas, as listed in Table 1, which are close to that of "CO nova 4." Therefore, we adopt the chemical composition of $X=0.55, Y=0.23, X_{\mathrm{CNO}}=0.2$, and $Z=0.02$ ("CO nova 4"). We made light curves of free-free plus blackbody emission for four different WD masses, $M_{\mathrm{WD}}=0.51,0.55,0.6$, and $0.65 M_{\odot}$, because the transition occurs from static to wind evolution for $0.5 M_{\odot} \lesssim M_{\mathrm{WD}} \lesssim 0.7 M_{\odot}$. The absolute magnitudes of free-free emission light curves are taken from Table 4 for the $0.55,0.6$, and $0.65 M_{\odot}$ WD models. The $0.51 M_{\odot}$ WD model is not tabulated in Table 4 but is calibrated in the same way as those of $0.55,0.6$, and $0.65 M_{\odot}$ WD models. We could not successfully obtain wind solutions for $M_{\mathrm{WD}} \leq 0.50 M_{\odot}$ because of numerical difficulty (Kato \& Hachisu 1994). Our results are shown in Figure 19 and 20 for RR Pic, in Figures 21, 22, and 23 for V5558 Sgr, in Figures 24 and 25 for HR Del, in Figures 26 and 27 for V723 Cas.

\subsection{RR Pic 1925}

RR Pic was discovered by Watson at about 2.3 mag on UT 1925 May 25. The details of visual light curve of RR Pic were found in Spencer Jones (1931), in which the photographic magnitude prior to 1925 was $m_{\mathrm{pg}}=12.75$ and the nova brightened up to 3rd magnitude between February 18 (fainter than 11th mag) and April 13 (3rd mag), and the first maximum was reached on UT June $7\left(m_{v}=1.18\right)$. Spencer Jones (1931) discussed a possibility of another peak between April 13 (3rd $\mathrm{mag})$ and May 25 (2.3 mag) before the first peak on June 7 $\left(m_{v}=1.18\right)$. Such a multiple peak has been observed also in V5558 Sgr, HR Del, and V723 Cas, as clearly shown in Figure 1. We superpose these four novae in Figure 3 to confirm that these novae have very similar decline rates. We shift horizontally their times and vertically their magnitudes by $+5.3,0.0$, +3.6 , and $+0.1 \mathrm{mag}$, respectively, as indicated in the figure. We also superpose these four novae in a logarithmic timescale in Figure 4. These four nova light curves almost overlap each other.

The distance to RR Pic was obtained using the trigonometric parallax, i.e., $d=521_{-45}^{+54} \mathrm{pc}$ (Harrison et al. 2013). The distance modulus in $V$ band is calculated to be $(m-M)_{V}=$ $5 \log 521_{-45}^{+54} / 10+0.13=8.7 \pm 0.2$, where we used $A_{V}=0.13$ after Harrison et al. (2013). Adopting this distance modulus, we plot, in Figure 19, our model light curves for four WD masses, i.e., (a) $0.51 M_{\odot}$, (b) $0.55 M_{\odot}$, (c) $0.60 M_{\odot}$, and (d) $0.65 M_{\odot}$ as well as the visual observation. Here, we assumed that the transition was completed at the first peak (UT 1925 June 7) and the outburst day was JD 2,424,170.0 about 140 days before the first peak. Figure 20 shows the same model light curves as those in Figure 19, but only the total $V$ light curves of different WD masses.

It is remarkable that two models of (a) $0.51 M_{\odot}$ and (b) $0.55 M_{\odot}$ nicely fit with the visual magnitude until the nebular phase begins about 450 days after the outburst ( $\sim 300$ days after the first peak, see Spencer Jones 1931; Iijima 2006). Note that the nova outburst begins quasi-statically and then undergoes a transition from static to wind evolution at the first peak. We think that, during the transition, the nova accompanies oscillatory activity of relaxation. This corresponds to the second and third peaks of the light curve. Thus, we confirm that the absolute magnitudes of our model light curves (total flux of free-free plus blackbody) are consistent with that of very slow novae even if they do not follow the universal decline law. Therefore, we confidently apply our absolute magnitude estimate to very slow novae.

It should be noted that the peak brightness of each model light curve depends on the initial envelope mass, which is closely related to the ignition mass of outburst. The larger the envelope mass is, the brighter the peak is. Therefore, we can estimate the envelope mass by adjusting the peak brightness to the observation if the WD mass is fixed.

Our model light curves of total $V$ flux have a similar brightness in the early phase of outburst for the WD mass range of $0.5 M_{\odot} \lesssim M_{\mathrm{WD}} \lesssim 0.7 M_{\odot}$ (see Figure 20). This property also can be seen in the light curve analysis of the slow nova 
Table 3

Physical parameters of the present models

\begin{tabular}{lllllllll}
\hline \hline object & $\begin{array}{c}\text { WD mass } \\
\left(M_{\odot}\right)\end{array}$ & $E(B-V)$ & $(m-M)_{V}$ & $\begin{array}{c}\text { distance } \\
(\mathrm{kpc})\end{array}$ & chem.comp. $^{\mathrm{a}}$ & $m_{V, \text { max }}$ & $\begin{array}{c}t_{2} \\
(\text { day })\end{array}$ & $\begin{array}{c}t_{3} \\
(\text { day })\end{array}$ \\
\hline PW Vul & 0.83 & 0.55 & 13.0 & 1.8 & CO Nova 4 & $6.3^{\mathrm{b}}$ & $82^{\mathrm{b}}$ & $126^{\mathrm{b}}$ \\
V705 Cas & 0.78 & 0.45 & 13.4 & 2.6 & CO Nova 4 & $5.5^{\mathrm{c}}$ & $33^{\mathrm{c}}$ & $61^{\mathrm{c}}$ \\
GQ Mus & 0.65 & 0.45 & 15.7 & 7.3 & CO Nova 2 & $7.2^{\mathrm{d}}$ & $18^{\mathrm{e}}$ & $40^{\mathrm{e}}$ \\
GQ Mus & 0.75 & 0.45 & 15.7 & 7.3 & CO Nova 4 & 7.2 & 18 & 40 \\
RR Pic & $0.5-0.60$ & $0.04^{\mathrm{f}}$ & 8.7 & $0.52^{\mathrm{f}}$ & CO Nova 4 & $1.1^{\mathrm{f}}$ & $78^{\mathrm{f}}$ & $136^{\mathrm{f}}$ \\
V5558 Sgr & $0.5-0.55$ & 0.70 & 13.9 & 2.2 & CO Nova 4 & $6.5^{\mathrm{g}}$ & $125^{\mathrm{h}}$ & $170^{\mathrm{g}}$ \\
HR Del & $0.5-0.55$ & 0.15 & 10.4 & 0.97 & CO Nova 4 & $3.76^{\mathrm{b}}$ & $172^{\mathrm{b}}$ & $230^{\mathrm{b}}$ \\
V723 Cas & $0.5-0.55$ & 0.35 & 14.0 & 3.9 & CO Nova 4 & $7.1^{\mathrm{i}}$ & $(102)^{\mathrm{i}}$ & $173^{\mathrm{i}}$ \\
\hline
\end{tabular}

a Chemical composition: see Table 2

${ }^{\mathrm{b}}$ Downes \& Duerbeck (2000)

${ }^{c}$ Hric et al. (1998)

$\mathrm{d}$ Warner (1995)

e Whitelock et al. (1984)

$\mathrm{f}$ Harrison et al. (2013)

$\mathrm{g}$ Poggiani (2010)

h Schwarz et al. 2011)

i Chochol \& Pribulla (1997)
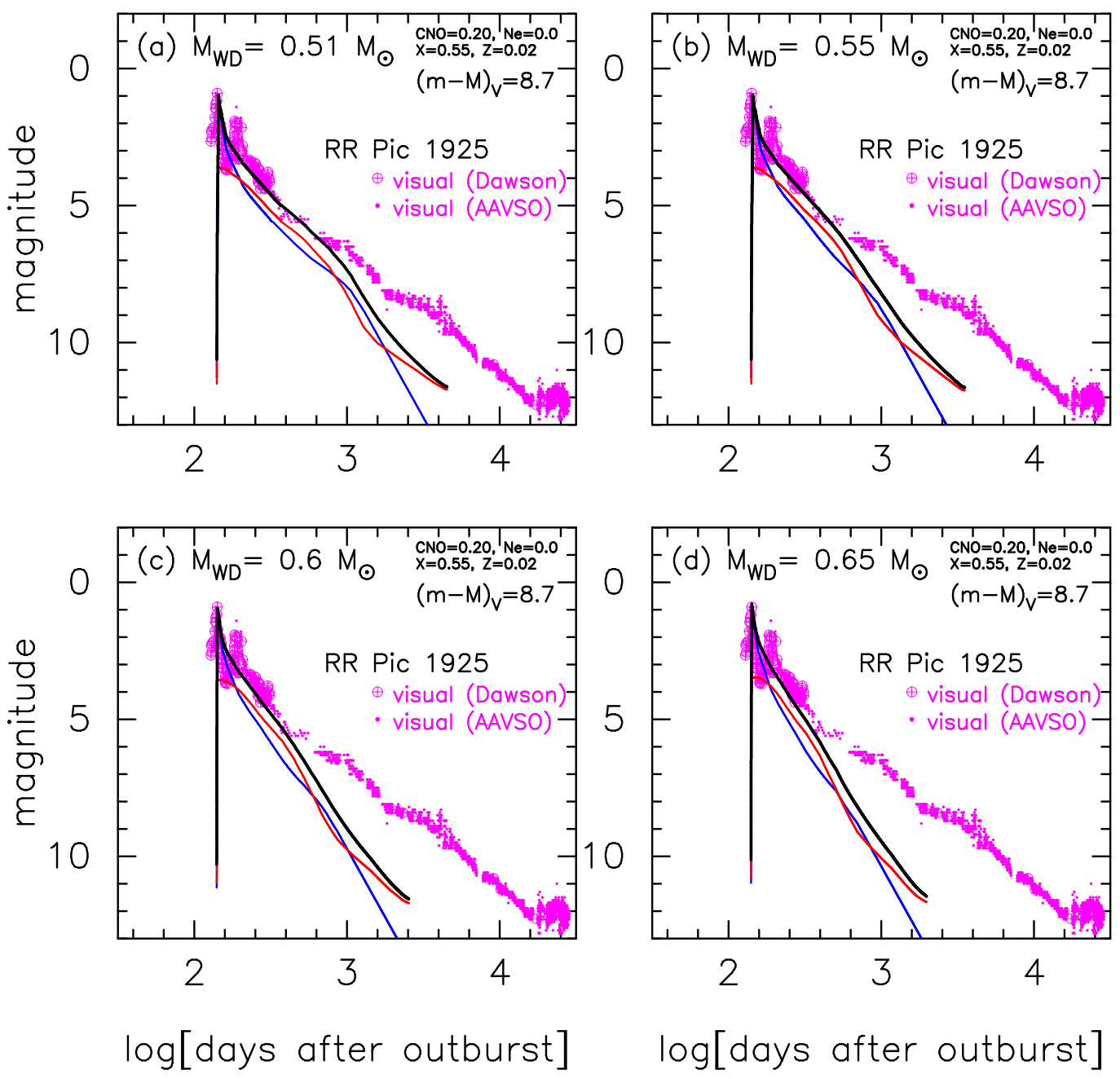

Figure 19. Visual light curves of RR Pic. Magenta circles with plus sign inside: visual magnitudes taken from Dawson (1926). Magenta dots: visual magnitudes taken from the AAVSO archive. We plot four different WD mass models: (a) $0.51 M_{\odot}$, (b) $0.55 M_{\odot}$, (c) $0.6 M_{\odot}$, and (d) $0.65 M_{\odot}$ for the envelope chemical composition of "CO nova 4." Blue solid lines denote the $V$ fluxes of free-free emission. Red solid lines represent the $V$ fluxes of blackbody emission. Black solid lines indicate the $V$ fluxes of total (free-free plus blackbody) emission. 


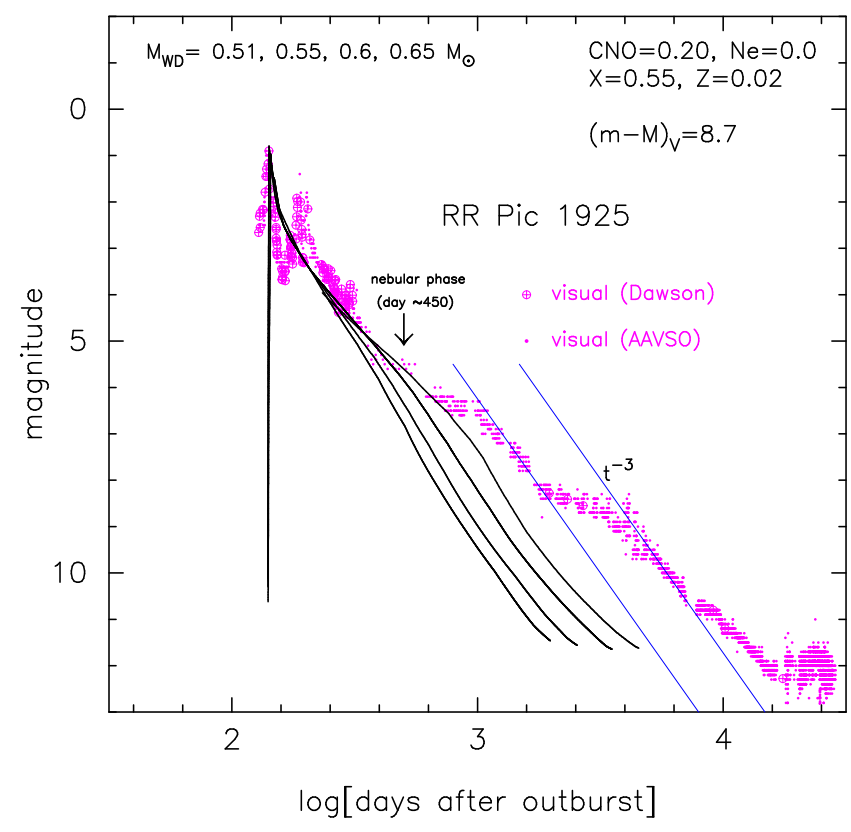

Figure 20. Same as Figure 19 but only for the total (free-free plus blackbody) $V$ flux model light curves. Black thick solid lines: total $V$ flux light curves for the $0.51,0.55,0.60$, and $0.65 M_{\odot}$ WD models.

DQ Her (see Figure 30 below). This is a problem in our light curve analysis because we are not able to identify the WD mass only from our model $V$ light curve fitting in the early phase. On the other hand, we are able to estimate the absolute magnitudes of slow novae, independently of the WD mass, by directly comparing them with a nova with known distance such as RR Pic.

Using the distance modulus of RR Pic, i.e., $(m-M)_{V}=8.7$, we obtain the distance moduli for the other three novae. Because these four novae have very similar decline shapes and should have similar brightnesses in the early phase of outbursts, we simply assumed that their brightnesses are all the same in the overlapping region of the light curves in Figures 3 and 4 . The difference in apparent $V$ magnitude against V723 Cas is -5.3 for RR Pic, -3.6 for HR Del, and -0.1 for V5558 Sgr. Therefore, the difference $\Delta V$ from RR Pic is calculated as $\Delta V=-3.6+5.3$ for HR Del, $\Delta V=-0.0+5.3$ for V723 Cas, and $\Delta V=-0.1+5.3$ for V5558 Sgr. Thus we have

$$
\begin{aligned}
(m-M)_{V, \mathrm{RR} \text { Pic }} & =8.7 \\
& =(m-M)_{V, \mathrm{HR} \mathrm{Del}}-\Delta V \\
& =10.4-(-3.6+5.3)=8.7 \\
& =(m-M)_{V, \mathrm{~V} 723 \mathrm{Cas}}-\Delta V \\
& =14.0-(-0.0+5.3)=8.7 \\
& =(m-M)_{V, \mathrm{~V} 5558 \mathrm{Sgr}}-\Delta V \\
& =13.9-(-0.1+5.3)=8.7 .
\end{aligned}
$$

Then, the distance moduli of these three novae are $(m-M)_{V, \mathrm{HR} \text { Del }}=10.4,(m-M)_{V, \mathrm{~V} 723 \mathrm{Cas}}=14.0$, and $(m-$ $M)_{V, \mathrm{~V} 5558 \mathrm{Sgr}}=13.9$.

\subsection{V5558 Sgr 2007}

V5558 Sgr was first detected by Sakurai (Nakano et al. 2007) at mag 10.3 on UT 2007 April 14.777. Sakurai also reported that nothing is visible on an image taken on UT April 9.8 (limiting mag 11.4). The star was also detected by Haseda (Yamaoka et al. 2007) at mag 11.2 on UT April 11.792. Since the outburst day is not known, we adopted UT 2007 April 8.5 as the outburst day, i.e., $t_{\mathrm{OB}}=\mathrm{JD} 2454199.0$,

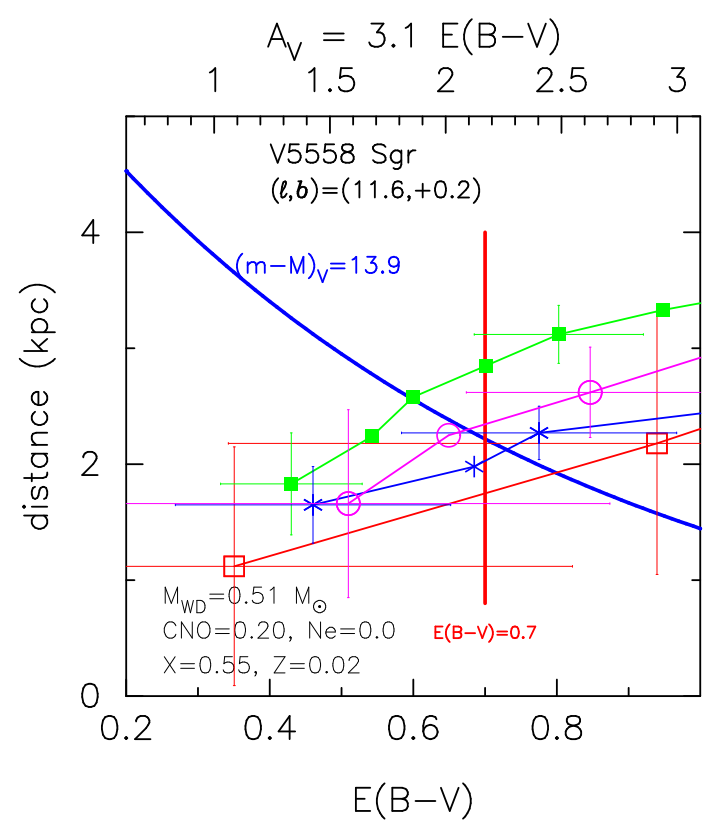

Figure 21. Various distance-reddening relations toward V5558 Sgr, whose galactic coordinates are $(l, b)=(11.6107,+0.2067)$. We plot distancereddening relations, which are taken from Marshall et al. (2006), in four directions close to V5558 Sgr. Red open squares: toward $(l, b)=(11.95,0.0)$, Green filled squares: toward $(11.75,0.0)$. Blue asterisks: toward $(11.5,0.25)$. Magenta open circles: toward $(11.75,0.25)$. We also plot $E(B-V)=0.7$ (vertical red solid line) and Equation (13), i.e., $(m-M)_{V}=13.9$ (blue solid line).

in this paper. The distance modulus in $V$ band was already obtained to be

$$
\begin{aligned}
(m-M)_{V} & =13.9 \\
& =5 \log \left(\frac{d}{10 \mathrm{pc}}\right)+3.1 \times E(B-V),
\end{aligned}
$$

in Equation (12) of Section 6.1. We plot this distancereddening relation of Equation (13) in Figure 21 We found in literature two different values of reddening, one is $E(B-V)=0.36$ obtained by Munari et al. (2007) from $\mathrm{Na} I \mathrm{D}$ lines and the other is $E(B-V)=0.8$ obtained by Rudy et al. (2007b) from O I lines. Since these two values are largely different, we examine other reddening estimates. Figure 21 also shows distance-reddening relations taken from Marshall et al. (2006) in four directions close to V5558 Sgr, $(l, b)=(11.6107,+0.2067)$. The closest one is that of blue asterisks, which crosses our line of $(m-M)_{V}=13.9$ at $E(B-$ $V) \approx 0.7$ and $d \approx 2.2 \mathrm{kpc}$. This reddening value is consistent with $E(B-V)=0.7 \pm 0.05$ estimated by Hachisu \& Kato (2014), who obtained the reddening by assuming that the three novae, V5558 Sgr, HR Del, and V723 Cas have the same intrinsic $(B-V)_{0}$ color in the premaximum phase. We adopt $(m-M)_{V}=13.9, E(B-V)=0.7$, and $d=2.2 \mathrm{kpc}$ in this paper.

Figure 22 shows optical and NIR light curves of V5558 Sgr and our model light curves for (a) 0.51 , (b) 0.55 , (c) 0.6 , and (d) $0.65 M_{\odot}$ WDs on a logarithmic timescale. Here, we assumed the distance modulus of $(m-M)_{V}=13.9$. Blue solid lines show the total $V$ fluxes (labeled "TOTAL") of our model light curves while green solid lines correspond to the free-free $V$ fluxes (labeled "FF") and black solid lines represent the blackbody $V$ fluxes (labeled "BB"). From the $V$ light curve shape, we assumed that the transition occurred from static to wind evolution about 90 days after the outburst (at the first optical peak). For comparison, we add another case of the 

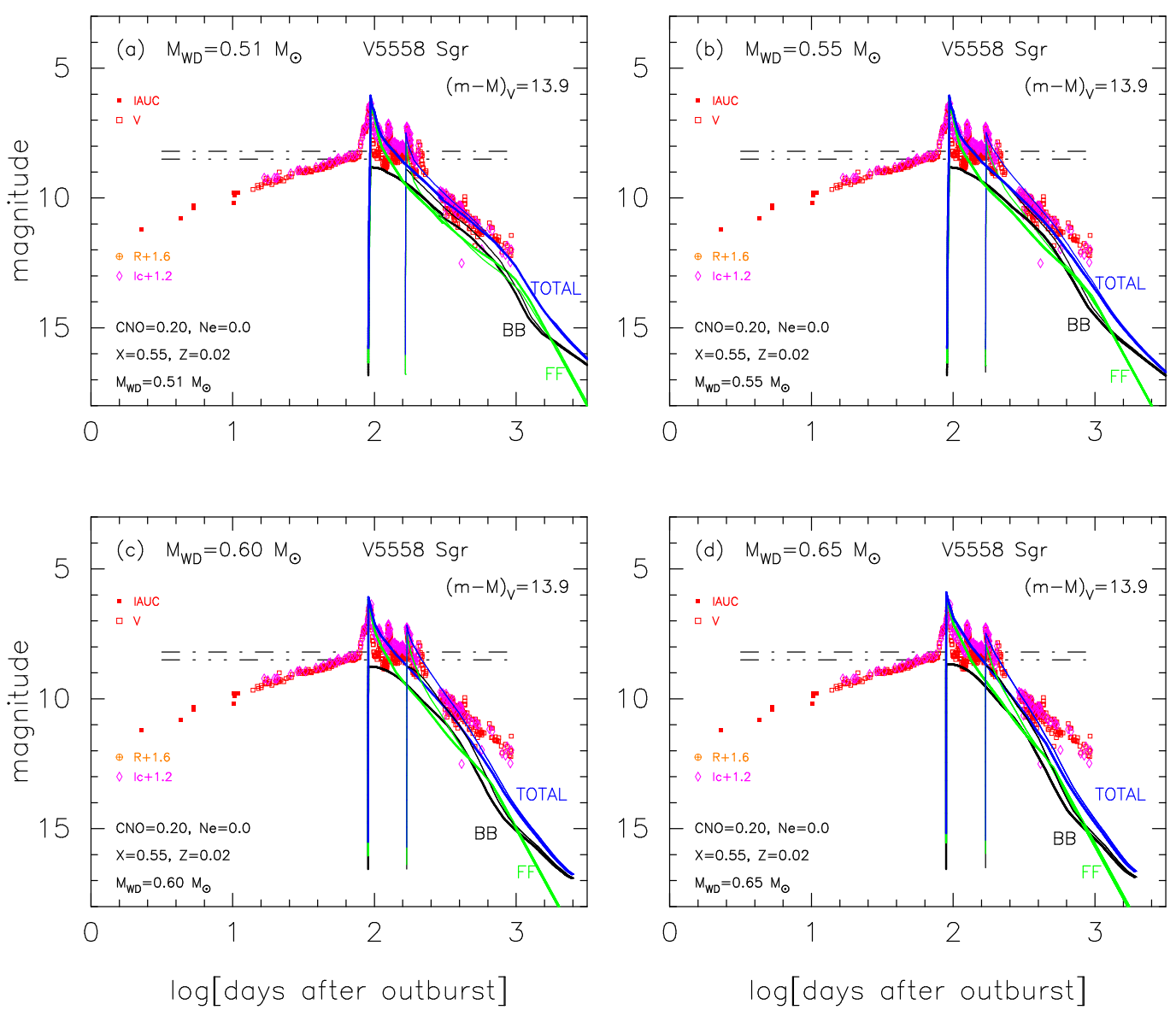

Figure 22. Optical and NIR light curves of V5558 Sgr. We assumed the distance modulus of $(m-M)_{V}=13.9$ from Equation (12). We plot four different WD mass models: (a) $0.51 M_{\odot}$, (b) $0.55 M_{\odot}$, (c) $0.6 M_{\odot}$, and (d) $0.65 M_{\odot}$ for the envelope chemical composition of "CO nova 4." Black solid lines labeled "BB" denote the $V$ fluxes of blackbody emission. Green solid lines labeled "FF" represent the $V$ fluxes of free-free emission. Blue solid lines labeled "TOTAL" depict the $V$ fluxes of total (free-free plus blackbody) emission. We assumed that the transition from static to wind evolution occurred just at the optical maximum (first peak). We also added different light curves for a different transition time at the third peak. Red filled squares: $V$ magnitudes taken from IAU Circular No. 8832 . Red open squares: $V$ magnitudes taken from archives of AAVSO and Variable Star Observers League of Japan (VSOLJ). Orange open circles with plus sign inside: $R_{C}$ magnitudes taken from the archives of AAVSO and VSOLJ. Magenta open diamonds: $I_{C}$ magnitudes taken from the archives of AAVSO and VSOLJ. Horizontal dash-dotted and dash-three-dotted lines denote the absolute magnitudes of $M_{V}=-5.7$ and -5.4 for each panel, which are the absolute magnitudes of the flat peak of the symbiotic nova PU Vul in 1979 and 1981-1983, respectively (see Figure 15 of Hachisu \& Kato 2014).

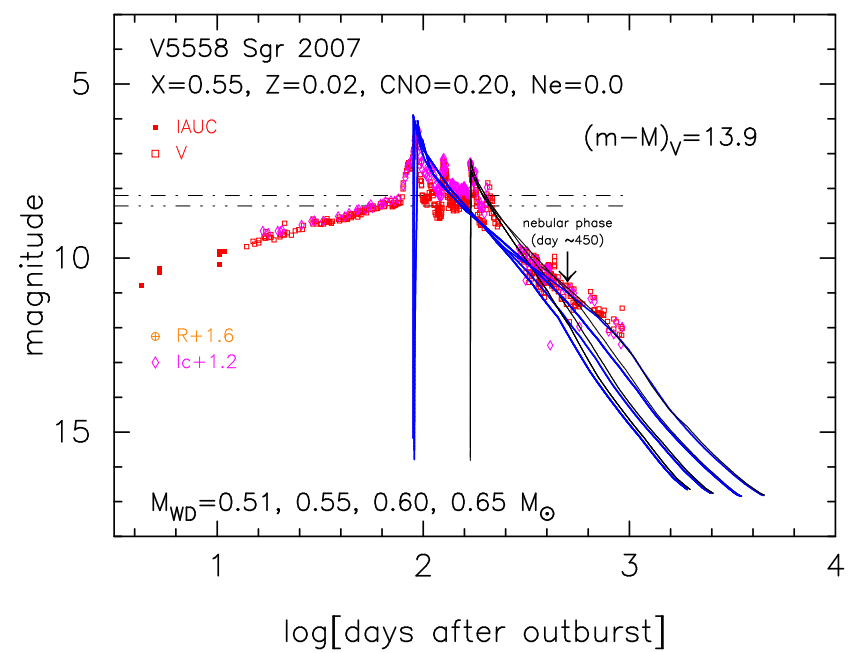

Figure 23. Same as Figure 22 but only for the total (free-free plus blackbody) $V$ flux model light curves. Blue thick solid lines: a transition occurs from static to wind evolution at the first peak. Black thin solid lines: a different transition time at the third peak.

transition at the third peak. Because the peak brightness of our model light curves depend on the initial envelope mass at the outburst, we tune the initial envelope mass to the peak brightness for each model. We adopted a less massive envelope for the model light curve of thin solid line that starts at the third peak of Figure 22. Figure 23 shows the same model light curves as those in Figure 22, but only the total $V$ light curves of different WD masses for comparison.

Among the four WD mass models, the 0.51 and $0.55 M_{\odot}$ WD models are in good agreement with the observation while the 0.60 and $0.65 M_{\odot}$ WDs may be too steep to be compatible with the observation. During the transition, the nova accompanies oscillatory activity of relaxation. This corresponds to the second, third, and fourth peaks of the light curve. This conclusion is unchanged even if we adopt the transition time at the third peak (thin solid lines in Figures 22 and 23). These fitting results are summarized in Table 3. This good agreement of the brightness supports that the absolute magnitudes of our model light curves (total flux of free-free plus blackbody) are reasonable even for very slow novae. It should be noted that the nebular phase started about 450 days after the outburst (a year after the fist peak, see Poggiani 2012). However, [O III] emission lines are too weak to contribute significantly to the $V$ flux, so that the $V$ light curve does not devi- 


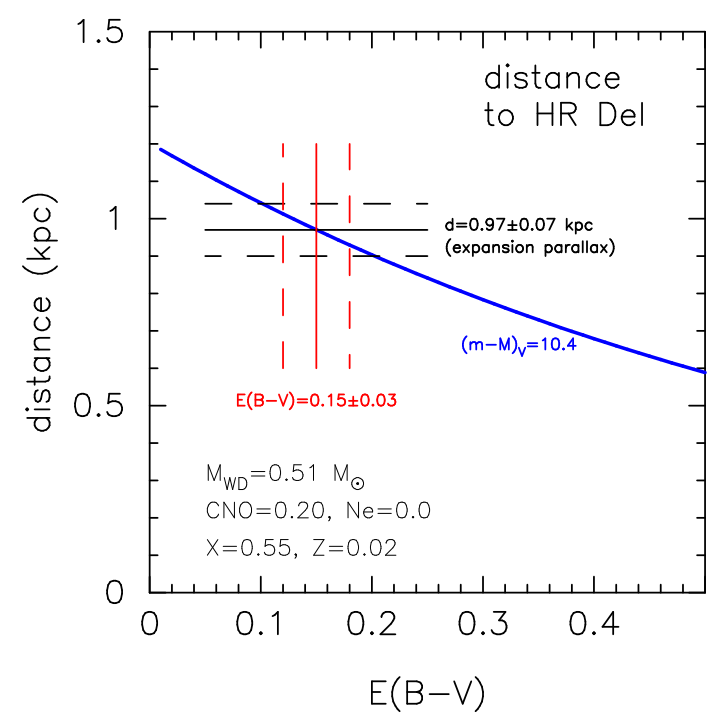

Figure 24. Distance-reddening relation toward HR Del. A blue solid line represents Equation (14), i.e., $(m-M)_{V}=10.4$. The distance and reddening estimates are taken from Harman \& O'Brien (2003) and Verbunt (1987), respectively.

ate so much from the model light curves as shown in Figures 22 (a) and 23 .

As already mentioned above, Kato \& Hachisu (2009, 2011) modeled the pre-maximum phase of these very slow novae with a static evolution followed by the transition from a static to a wind structure. They predicted that this transition occurs in a narrow range of WD masses, $0.5 M_{\odot} \lesssim M_{\mathrm{WD}} \lesssim$ $0.7 M_{\odot}$. Thus, the brightness at the pre-maximum phase of these novae should be similar to the flat peak of the symbiotic nova PU Vul $\left(M_{\mathrm{WD}} \sim 0.6 M_{\odot}\right)$, whose brightness is $M_{V}=-5.4$ in stage 1 (in 1979) and $M_{V}=-5.7$ in stage 2 (in 1981-1983), respectively (see Figure 15 of Hachisu \& Kato 2014). We plot these two absolute magnitudes of PU Vul in Figures 22 and 23 (horizontal thin dash-dotted and dashthree-dotted lines). These brightnesses are in perfect agreement with the brightness of V5558 Sgr at the premaximum halt (flat) phase just before the first peak, i.e., before the transition started.

\subsection{HR Del 1967}

The slow nova HR Del was discovered by Alcock (Candy et al. 1967) at $m_{v}=5.0$ on UT 1967 July 8.94 (JD 2439680.44 ). Since the outburst day is not known, we adopt UT 1967 June 8.5 as the outburst day, i.e., $t_{\mathrm{OB}}=\mathrm{JD} 2439653.0$, from the light curve of Robinson \& Ashbrook (1968). The distance modulus of HR Del was already obtained to be

$$
\begin{aligned}
(m-M)_{V} & =10.4 \\
& =5 \log \left(\frac{d}{10 \mathrm{pc}}\right)+3.1 \times E(B-V),
\end{aligned}
$$

in Equation (12) of Section 6.1 We plot this distancereddening relation of Equation (14) in Figure 24 by a blue solid line. Verbunt (1987) obtained the extinction toward HR Del to be $E(B-V)=0.15 \pm 0.03$. The galactic dust absorption map of NASA/IPAC gives $E(B-V)=0.11 \pm 0.006$ in the direction toward HR Del, whose galactic coordinates are $(l, b)=(63.4304,-13.9721)$, being roughly consistent with Verbunt's value. Two lines of $E(B-V)=0.15$ and Equation (14) cross at a distance of $d=0.97 \mathrm{kpc}$ as shown in Figure 24
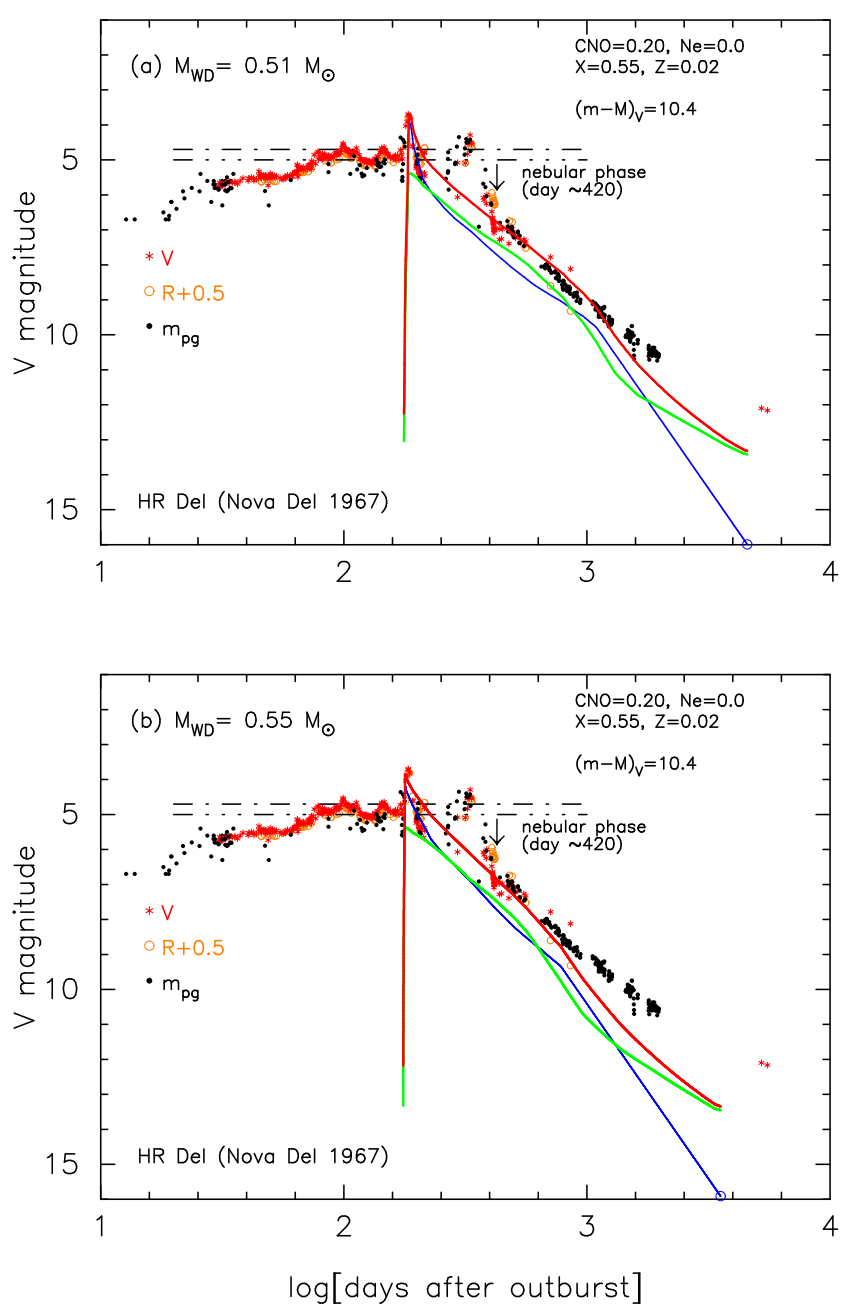

Figure 25. Optical light curves of HR Del. We plot two different WD mass models: (a) $0.51 M_{\odot}$ and (b) $0.55 M_{\odot}$ for the envelope chemical composition of "CO nova 4." We assumed that the transition occurred about 170 days after the outburst and that the distance modulus in $V$ band is $(m-M)_{V}=10.4$. Red asterisks: $V$ magnitudes. Orange open circles: $R$ magnitudes. Black filled circles: photographic magnitudes, $m_{\mathrm{pg}}$. Observational data are taken from IAU Circular Nos. 2024, 2025, 2030, 2036, and from Stokes (1967), Nha (1967), Onderlička \& Vetešník (1968), O’Connell (1968), Terzan (1968), Grygar (1969), Mollerus (1969), Mannery (1970), Barnes \& Evans (1970) and the AAVSO archive. Green solid lines indicate the blackbody $V$ flux, blue solid lines represent the free-free $V$ flux, and red solid lines denote the total $V$ flux of free-free plus blackbody. Horizontal dash-dotted and dash-threedotted lines denote the absolute magnitudes of $M_{V}=-5.7$ and -5.4 , which are the absolute magnitudes of the flat peak of PU Vul.

Downes \& Duerbeck (2000), on the other hand, obtained the distance to HR Del to be $d=0.76 \pm 0.13 \mathrm{kpc}$ from the nebular expansion parallax. More recently, Harman \& O'Brien (2003) obtained a new value of the distance $d=0.97 \pm$ $0.07 \mathrm{kpc}$ also from the expansion parallax method of HST imaging. Other older estimates are all between the above two estimates, i.e., $d=0.940 \pm 0.155 \mathrm{kpc}$ for various expansion parallax methods (Malakpur 1975; Kohoutek 1981; Duerbeck 1981; Solf 1983; Cohen \& Rosenthal 1983; Slavin et al. 1994, 1995) or $d=0.835 \pm 0.092 \mathrm{kpc}$ for the other techniques (Drechsel et al. 1977). Here we adopt the distance of $d=0.97 \pm 0.07 \mathrm{kpc}$ after Harman \& O'Brien (2003) and the extinction of $E(B-V)=0.15 \pm 0.03$ after Verbunt (1987). These two values are consistent with Equation (14) in Figure 24

We plot the light curve of HR Del in Figures 1 and 3 on 
a linear timescale and in Figures 4 and 25 on a logarithmic timescale. Figure 25 shows two model light curves for (a) $0.51 M_{\odot}$ and (b) $0.55 M_{\odot}$ WDs, in which we assumed that the transition completed 170 days after the outburst. Red solid lines show the total $V$ flux of free-free (blue solid lines) plus blackbody (green solid lines). We calculated four model light curves of $0.51,0.55,0.60$, and $0.65 M_{\odot}$ WDs but did not plot the 0.60 and $0.65 M_{\odot}$ WDs because these two are too steep to be compatible with the observation. The $0.51 M_{\odot}$ WD model shows good agreement with the observation while the $0.55 M_{\odot}$ WD model is marginal, as shown in Figure 25(b). Note that the nebular phase started about 420 days after the outburst ( $\sim 250$ days after the fist peak, see Iijima 2006). During the transition, the nova accompanies oscillatory activity of relaxation. This corresponds to the second peak of the light curve. The brightness of $M_{V}=-5.7$ in PU Vul is in good agreement with the brightness of HR Del at the premaximum halt (flat) phase just before the optical maximum, i.e., before the transition started. This fact also confirms that our absolute magnitudes of optical light curves for slow novae are reasonable.

\subsection{V723 Cas 1995}

V723 Cas is also a very slow nova. It was discovered at mag 9.2 by Yamamoto on UT 1995 August 24.57 (JD 2449954.07). Munari et al. (1996) proposed UT July 20.5 as the outburst day, i.e., $t_{\mathrm{OB}}=\mathrm{JD} 2449919.0$, so we adopt this day in this paper. We plot the visual, $V, R, I, J, H$, and $K$ light curves of V723 Cas in Figure 26 together with the UV $1455 \AA$ and X-ray light curves. The distance modulus of HR Del was already obtained to be

$$
\begin{aligned}
(m-M)_{V} & =14.0 \\
& =5 \log \left(\frac{d}{10 \mathrm{pc}}\right)+3.1 \times E(B-V),
\end{aligned}
$$

in Equation (12) of Section 6.1 We plot this distancereddening relation of Equation (15) in Figure 27 by a blue solid line. The distance to V723 Cas was estimated by Lyke \& Campbell (2009) to be $d=3.85_{-021}^{+0.23} \mathrm{kpc}$ from the expansion parallax method. Hachisu \& Kato (2014) obtained the reddening toward V723 Cas to be $E(B-V)=0.35 \pm$ 0.05 by fitting the general tracks with the observed track of V723 Cas in the $U B V$ color-color diagram. These three trends, i.e., $(m-M)_{V}=14.0, d=3.85 \mathrm{kpc}$, and $E(B-V)=0.35$, cross consistently as shown in Figure 27. Therefore, we adopt these values in this paper.

Figure 26 shows our model light curves of the $0.51 M_{\odot}$ WD for the chemical composition of "CO nova 4." Here, we assumed the distance modulus in $V$ band to be $(m-M)_{V}=14.0$ and that the transition from static to wind evolution occurred 155 days after the outburst, because the UV $1455 \AA$ flux started to rise on this day. A black solid line shows the total $V$ flux of free-free (blue solid line) plus blackbody (sky blue solid line) emission calculated from the $0.51 M_{\odot}$ WD model which is the one showing the best agreement with the observation compared with the other three WD mass models of 0.55 , 0.60 , and $0.65 M_{\odot}$. During the transition, the nova accompanies oscillatory activity of relaxation. This corresponds to the second, third, and fourth peaks of the light curve. Note that the model light curves were fitted to the lower envelope of the $V$ light curve to avoid local photospheric fluctuations until the nebular phase started about 700 days after the outburst $(\sim 550$ days after the fist peak, see Iijima 2006).
The following additional distance-reddening relation (labeled "UV $1455 \AA$ " in Figure 27) can be deduced from our UV $1455 \AA$ flux fitting, i.e.,

$$
\begin{aligned}
& 2.5 \log F_{1455}^{\mathrm{mod}}-2.5 \log F_{1455}^{\mathrm{obs}} \\
= & 2.5 \log \left(3.2 \times 10^{-12}\right)-2.5 \log \left(1.5 \times 10^{-12}\right) \\
= & 5 \log \left(\frac{d}{10 \mathrm{kpc}}\right)+8.3 \times E(B-V),
\end{aligned}
$$

where $F_{1455}^{\text {mod }}=3.2 \times 10^{-12}$ erg cm $\mathrm{cm}^{-2} \mathrm{~s}^{-1} \AA^{-1}$ is the calculated flux at the upper limit of the figure box at the distance

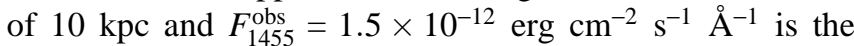
observed flux corresponding to the upper limit of the figure box. The two distance-reddening relations, i.e., Equation (15) and Equation (16), cross each other at the point of $E(B-V) \approx 0.34$ and $d \approx 3.9 \mathrm{kpc}$, being consistent with the distance of $d=3.85_{-0.21}^{+0.23} \mathrm{kpc}$ (Lyke \& Campbell 2009) and $E(B-V)=0.35 \pm 0.05$ (Hachisu \& Kato 2014) mentioned above.

Ness et al. (2008) obtained $(m-M)_{V}=13.7, E(B-V)=$ $0.5 \pm 0.1$, and $d=2.7_{-0.3}^{+0.4} \mathrm{kpc}$ by assuming that the absolute magnitude of V723 Cas is similar to that of HR Del. The main difference from ours comes from the reddening estimate. The interstellar extinction toward V723 Cas was estimated by many authors but the values are quite scattered; that is, in increasing order, $E(B-V)=0.20 \pm 0.12$ in August 1999 and $0.25 \pm 0.1$ in July 2000 (Rudy et al. 2002) from Paschen and Brackett lines, $E(B-V)=0.29$ calculated from $A_{V}=0.89$ (Iijima et al. 1998) from reddenings of field stars near the location of V723 Cas, $E(B-V)=0.45$ (Munari et al. 1996) from interstellar Na I D double lines, $E(B-V)=0.5 \pm 0.1$ (Ness et al. 2008) estimated from various values in literature and their $N_{\mathrm{H}}$ value from X-ray spectrum model fits, $E(B-V)=$ 0.57 (Chochol \& Pribulla 1997) from intrinsic colors at maximum and at two magnitude below maximum, $E(B-V)=0.60$ (González-Riestra et al. 1996) from the 2200A dust absorption feature, and $E(B-V)=0.78 \pm 0.15$ (Evans et al. 2003) from the IR H I recombination lines. Recently, GonzálezRiestra revised the value to be $E(B-V)=0.30-0.35$ (private communication 2012, see also Hachisu \& Kato 2014). Hachisu \& Kato (2014) obtained $E(B-V)=0.35 \pm 0.05$ by fitting the general tracks with the observed track of V723 Cas in the $U B V$ color-color diagram. The recent NASA/IPAC dust map gives $E(B-V)=0.34 \pm 0.01$ toward V723 Cas, whose galactic coordinates are $(l, b)=(124.9606,-8.8068)$. Therefore, we adopt $E(B-V)=0.35$ and $d=3.85 \mathrm{kpc}$ in this paper. These fitting results are summarized in Table 3

\section{DISCUSSION}

\subsection{Brightness Confirmation of Model Light Curves}

We examine whether or not the absolute brightness of our model light curve is correct for classical novae with known distances. Harrison et al. (2013) determined the distances of four novae, V603 Aql, GK Per, DQ Her, and RR Pic, with HST annual parallaxes. We have already examined the case of RR Pic in Section 6.1 and showed that the total brightness of our model light curve reasonably reproduces the absolute brightness of RR Pic for the distance modulus of $(m-M)_{V, \mathrm{RR} \text { Pic }}=8.7$ (Harrison et al. 2013). In this subsection, we study the other three, i.e., GK Per, V603 Aql, and DQ Her. 


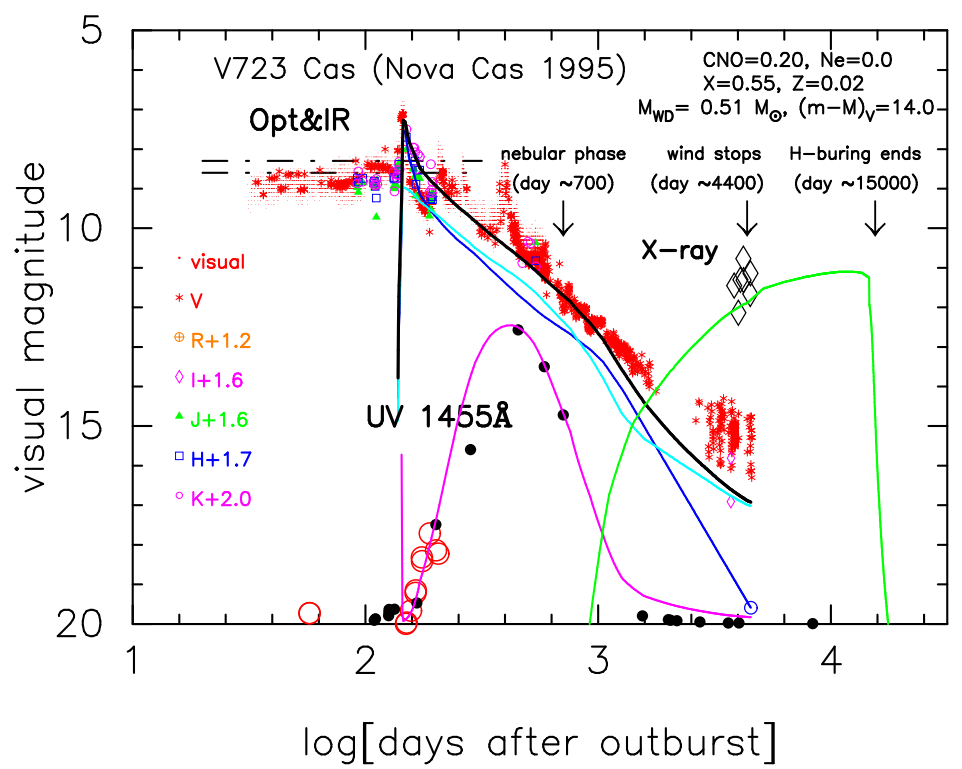

$\log [$ days after outburst]

Figure 26. Optical, NIR, UV $1455 \AA$, and supersoft X-ray light curves of V723 Cas. Large open red circles: IUE UV 1455 ̊ata (Cassatella et al. 2002). Large open black diamonds: supersoft X-ray fluxes of 0.25-0.6 keV taken from Ness et al. (2008). Other symbols show $V, R, I, J, H$, and $K$ observational data, which are taken from Chochol \& Pribulla (1997, 1998), Kamath \& Ashok (1999), and the AAVSO archive. Black filled circles: UV 1455 (Cassatella et al. 2002), but the timescale is stretched by 5.8 times. Horizontal dash-dotted and dash-three-dotted lines denote the absolute magnitudes of $M_{V}=-5.7$ and -5.4 of the flat peaks of PU Vul in 1979 and in 1981-1983, respectively. We plot the $0.51 M_{\odot}$ WD model for the envelope chemical composition of "CO nova 4," which are the free-free emission (thick blue solid line), blackbody emission (sky blue solid line), total of free-free plus blackbody (black solid line), UV 1455 (magenta solid line), and supersoft X-ray (calculated from blackbody emission; green solid line) light curves.

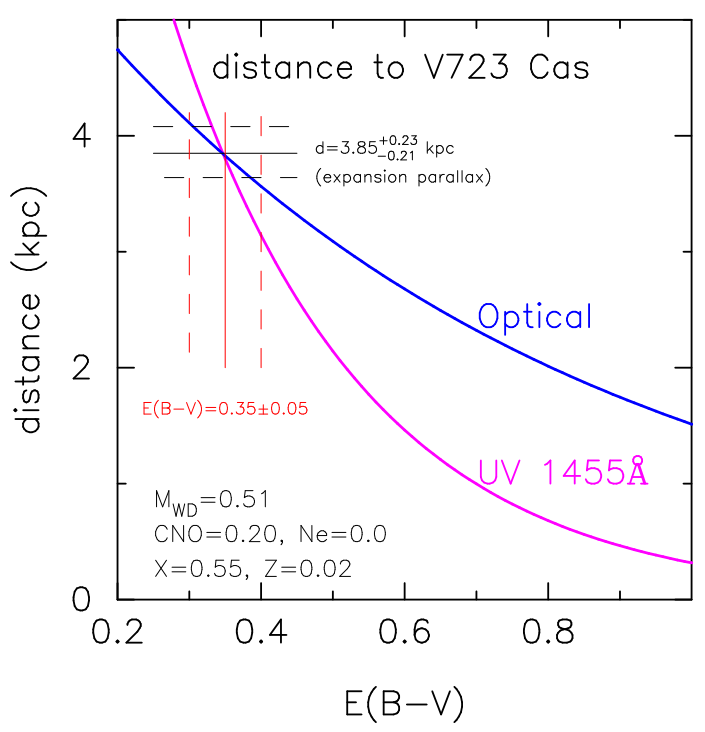

Figure 27. Distance-reddening relation toward V723 Cas. A blue solid line represents Equation [15, i.e., $(m-M)_{V}=14.0$. A magenta solid line depicts the UV $1455 \AA$ distance-reddening relation of Equation 16. The distance estimate is taken from Lyke \& Campbell (2009) and the color excess is taken from Hachisu \& Kato (2014).

\subsubsection{GK Per 1901}

The distances of GK Per is obtained to be $d=477_{-25}^{+28} \mathrm{pc}$ by Harrison et al. (2013). The distance modulus in $V$ band is obtained to be $(m-M)_{V, \mathrm{GK} \text { Per }}=5 \log 477_{-25}^{+28} / 10+3.1 \times$ $0.3=9.3 \pm 0.1$, where we adopt $E(B-V)=0.3$ (Wu et al. 1989) after Harrison et al. (2013). The WD mass of GK Per was estimated by Morales-Rueda et al. (2002) to be $M_{\mathrm{WD}}=$ $0.77_{-0.24}^{+0.52} M_{\odot}$, being not accurately constrained.

For GK Per, we assumed the chemical composition of "Ne nova 2 " because no estimates are available in literature. For this chemical composition, the absolute magnitudes of freefree emission model light curves were already determined in Table 3 of Hachisu \& Kato (2010). Using the absolute magnitudes of free-free model light curves, we calculated the total (free-free plus photospheric) $V$ flux light curves for the WD masses of $1.05,1.1,1.15$, and $1.2 M_{\odot}$. We plot these four $V$ light curves in Figure 28 a), where we adopted $(m-M)_{V, \mathrm{GK} \text { Per }}=9.3$. Among the four WD masses, we obtained a best fit for $M_{\mathrm{WD}}=1.15 M_{\odot}$ (a thick black solid line). The other fluxes (blackbody and free-free fluxes) are also plotted only for $M_{\mathrm{WD}}=1.15 M_{\odot}$ in Figure 28(b).

In Figure 28, we plot optical and NIR light curves of the very fast nova V1500 Cyg as well as the fast nova GK Per. GK Per shows a transition phase in the middle part of the outburst. We do not know how to fit our model light curves with the observation in such an oscillatory light curve. For this purpose, we overlap the light curve of V1500 Cyg to that of GK Per and select which part of oscillatory brightness to be fitted. In the figure, we shift the light curve of GK Per horizontally by $\Delta \log t=-0.20$ and vertically $\Delta V=+2.6$ mag to overlap it to the light curves of V1500 Cyg. The two $V$ light curves reasonably overlap in the early phase and in the very later phase. In the middle part of the light curves, the lower bound of oscillatory brightness of GK Per reasonably overlaps that of V1500 Cyg. Therefore, we fit our model light curves to the lower bound of oscillatory brightness during the transition phase of GK Per. Note that our model light curve reasonably fits with the early $V$ light curve but deviates from the visual observation in the nebular phase. This deviation in visual magnitudes is owing to strong emission lines such as [O III], which are not included in our model (see Hachisu \& Kato 2006, for details).

To summarize, we are able to reproduce the absolute brightness for the distance modulus of $(m-M)_{V, \mathrm{GK} \text { Per }}=9.3$ and $M_{\mathrm{WD}}=1.15 M_{\odot}$. Photospheric emission (red solid line labeled "BB") does not contribute to the total $V$ flux (black 

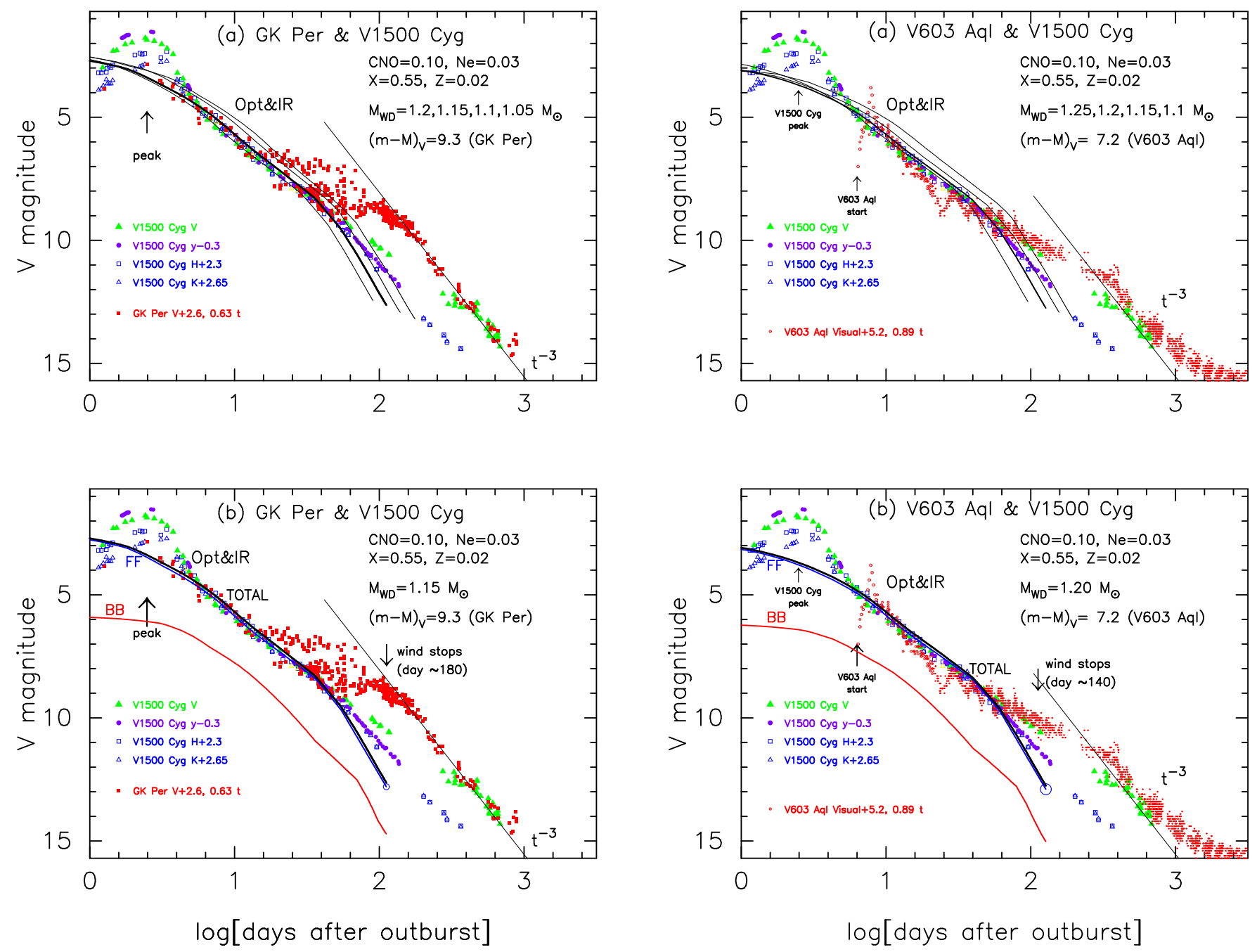

Figure 28. Light curves of GK per in a logarithmic timescale. The light curves of V1500 Cyg are also added and overlapped to those of GK Per. The light curves of GK Per are shifted horizontally by $\Delta \log t=$ -0.20 leftward and vertically by $\Delta V=+2.6$ downward. Visual observations (red filled squares) of GK Per are taken from Child (1901), Gore (1901), Rambaut (1901a b c d e, 1902, 1903), Sharp (1901), and Williams (1901a b c d, 1902, 1919). For V1500 Cyg, the $y$ magnitudes are taken from Lockwood \& Millis (1976), the $V$ magnitudes are from Gallagher \& Nev (1976), Arkhipova \& Zaitseva (1976), Pfau (1976), and Tempesti (1979), the NIR $H$ and $K$ magnitudes are taken from Gallagher \& Nev (1976), Kawara et al. (1976), and Ennis et al. (1977). (a) Assuming the chemical composition of the envelope "Ne nova 2," we plot four total $V$ flux light curves for the WD mass models of $1.05,1.1,1.15$, and $1.2 M_{\odot}$. Among these four nova light curves, we found the $1.15 M_{\odot}$ (thick black solid line) as a best fit model. (b) We plot three $V$ model light curves of photospheric (blackbody, red solid line labeled "BB"), free-free (blue solid line labeled "FF"), and total flux (black solid line labeled "TOTAL). The photospheric emission does not much contribute to the total $V$ flux.

solid line labeled "TOTAL") as shown in the Figure 28(b). Hachisu \& Kato (2006) showed that nova light curves follow a universal decline law if free-free emission dominates the spectrum. Figure 28(b) confirms that the nova light curves follow the universal decline law.

\subsubsection{V603 Aql 1918}

The distance of V603 Aql is obtained to be $d=249_{-8}^{+9} \mathrm{pc}$ by Harrison et al. (2013). The distance modulus in $V$ band is calculated to be $(m-M)_{V, \mathrm{~V} 603 \mathrm{Aql}}=5 \log 249_{-8}^{+9} / 10+$ $3.1 \times 0.07=7.2 \pm 0.07$, where we adopt $E(B-V)=0.07$ (Gallagher \& Holm 1974) after Harrison et al. (2013). The

Figure 29. Same as Figure 28 but for V603 Aql. Visual data of V603 Aql are taken from Campbell \& Shapley (1923) (red small open circles) and from AAVSO (red small dots). The light curves of V603 Aql are shifted horizontally by $\Delta \log t=-0.05$ leftward and vertically by $\Delta V=+2.4$ downward. (a) Assuming the chemical composition of the envelope "Ne nova 2," we plot four total $V$ flux light curves for the WD mass models of 1.1, 1.15, 1.2, and $1.25 M_{\odot}$. Among these four nova light curves, we found the $1.2 M_{\odot}$ (thick black solid line) as a best fit model. (b) We plot three $V$ model light curves of photospheric (blackbody, red solid line labeled "BB"), free-free (blue solid line labeled "FF"), and total flux (black solid line labeled "TOTAL) for the best-fit $1.2 M \odot$ WD. The photospheric emission also does not much contribute to the total $V$ flux.

WD mass of V603 Aql was obtained by Arenas et al. (2000) to be $M_{\mathrm{WD}}=1.2 \pm 0.2 M_{\odot}$.

The chemical composition of V603 Aql is not available, so we assume "Ne nova 2 " in this paper partly because we already estimated the absolute magnitudes of free-free emission model light curves for "Ne nova 2" (Hachisu \& Kato 2010). We calculated the total (free-free plus photospheric) $V$ flux light curves for the WD masses of 1.1, 1.15, 1.2, and $1.25 M_{\odot}$. We plot these four $V$ light curves in Figure 29(a), where we adopted $(m-M)_{V, V 603}$ Aql $=7.2$. Among the four WD masses, we obtained a best fit for $M_{\mathrm{WD}}=1.2 M_{\odot}$ (a thick black solid line). The other fluxes (blackbody and free-free fluxes) are also plotted only for $M_{\mathrm{WD}}=1.2 M_{\odot}$ in Figure 29 b). The brightness of our model light curves are consistent with both the distance modulus of $(m-M)_{V}=7.2$ and the WD mass of $M_{\mathrm{WD}}=1.2 M_{\odot}$. This confirms that the absolute magnitudes 
of our model light curves are reasonable.

In Figure 29, we add optical and NIR light curves of the very fast nova V1500 Cyg. V603 Aql shows a transition phase in the middle part of the outburst like GK Per. In order to know how to fit our model light curves with the oscillatory light curve, we again overlap the light curve of V1500 Cyg to that of V603 Aql and select which part of oscillatory brightness to be fitted. In the figure, we shift the light curve of V603 Aql horizontally by $\Delta \log t=-0.05$ and vertically $\Delta V=+5.2 \mathrm{mag}$ to overlap it to the light curves of V1500 Cyg. Moreover, we set the start of the light curves about 7 days later (indicated by an arrow) than the start of V1500 Cyg. The two $V$ light curves reasonably overlap in the early phase and in the very later phase. In the middle part of the light curves, the upper bound of oscillatory brightness of V603 Aql reasonably overlaps that of V1500 Cyg. Therefore, we fit our model light curves to the upper bound of oscillatory brightness during the transition phase of V603 Aql. Again note that our model light curve reasonably fits with the early $V$ light curve but deviates from the visual observation in the later nebular phase.

To summarize, we are able to reproduce the absolute brightness for the distance modulus of $(m-M)_{V, V 603 \text { Aql }}=7.2$ and $M_{\mathrm{WD}}=1.20 M_{\odot}$. Photospheric emission does not contribute to the total $V$ flux as shown in the Figure 29 b).

\subsection{3. $D Q$ Her 1934}

The trigonometric parallax distance of DQ Her is $d=$ $386_{-29}^{+33} \mathrm{pc}$ (Harrison et al. 2013). Adopting $A_{V}=3.1 \times E(B-$ $V)=3.1 \times 0.1=0.31$ (Verbunt 1987), we obtain the distance modulus in $V$ band as $(m-M)_{V}=A_{V}+5 \log (d / 10 \mathrm{pc})=$ $0.31+5 \log \left(386_{-29}^{+33} / 10\right)=8.24 \pm 0.18$. Thus, we adopt $(m-$ $M)_{V . \mathrm{DO} H e r}=8.2$. The WD mass of DQ Her was obtained by Horne et al. (1993) to be $M_{\mathrm{WD}}=0.60 \pm 0.07 M_{\odot}$. The chemical composition of ejecta was estimated by Petitjean et al. (1990) and Williams et al. (1978) as listed in Table 1. Here, we adopt the chemical composition of "CO nova 2" because the averaged value is $X=0.31$ and close to that of "CO nova 2."

For the chemical composition of "CO nova 2," the absolute magnitudes of free-free emission model light curves were already determined in Table 2 of Hachisu \& Kato (2010). So we calculated the total (free-free plus photospheric) $V$ flux light curves for the WD masses of $0.55,0.60,0.65$, and $0.70 M_{\odot}$ and plotted them in Figure 30 a). It is remarkable that all the four $V$ light curves fit reasonably to the observed visual magnitudes, at least, in the early phase before the dust blackout started. Therefore, we cannot select a best one among these four light curves. However, this again confirms that the absolute brightness of our model light curves are reasonable at least in the early decline phase before the dust blackout.

In Figure 30(b), we adopt $M_{\mathrm{WD}}=0.60 M_{\odot}$ from the central value estimated by Horne et al. (1993) and plot our total $V$ flux, free-free, and photospheric blackbody light curves for the $0.6 M_{\odot}$ WD. The photospheric emission (red solid line labeled "BB") significantly contribute to the total $V$ flux (black solid line labeled "TOTAL"). If we do not include the photospheric emission, our free-free model $V$ flux (blue solid line labeled "FF") does not fit to the observed one. This good agreement with the observed brightness suggests that photospheric emission is necessary to reproduce the light curves of slow novae like DQ Her as well as free-free emission.
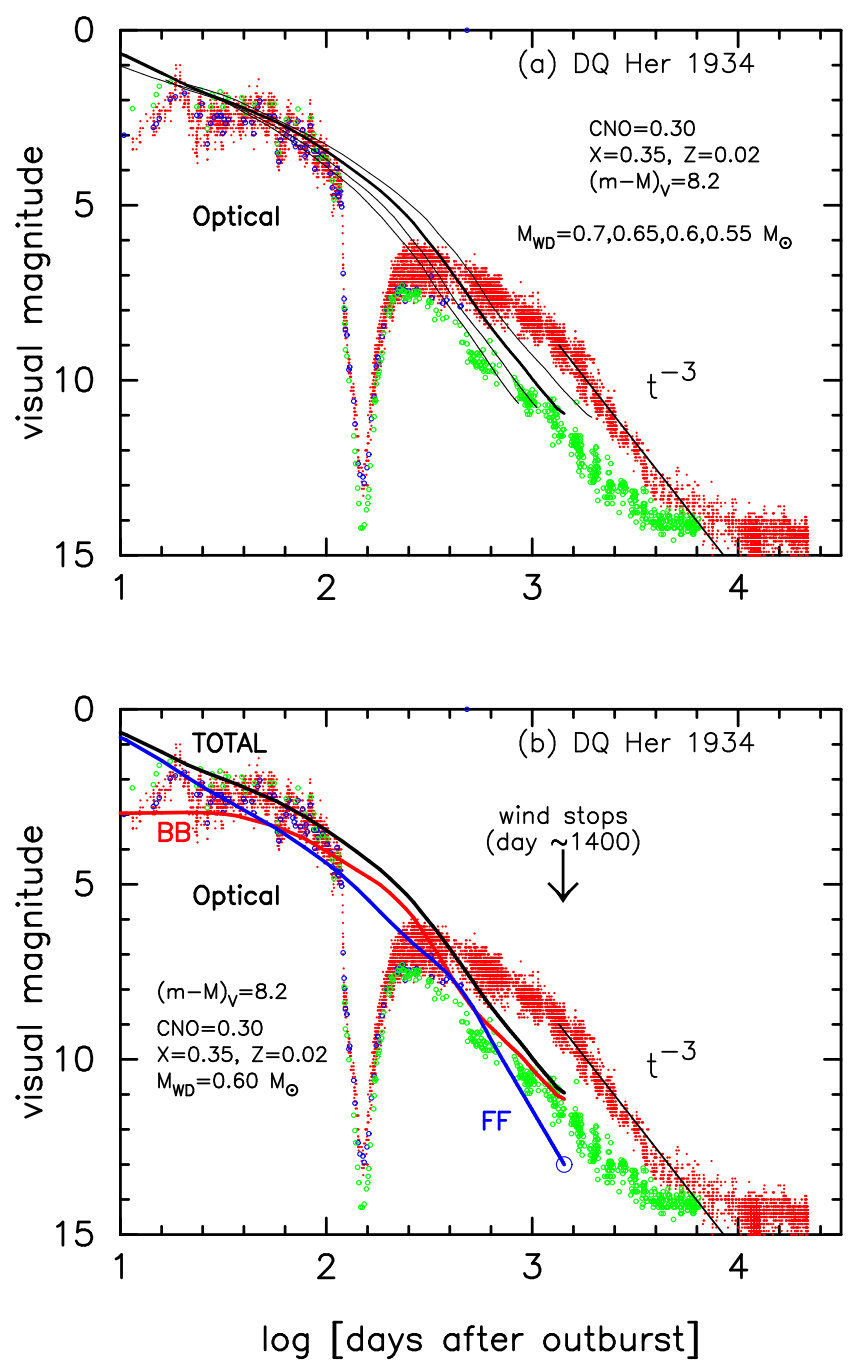

Figure 30. Same as Figure 28, but for DQ Her. Visual magnitudes of DQ Her are taken from AAVSO (red small dots) and Gaposchkin (1956) (blue small open circles), and photographic magnitudes (green small open circles) are from Gaposchkin (1956). (a) Assuming the chemical composition of the envelope "CO nova 2," we plot four total $V$ flux light curves for the WD mass models of $0.55,0.6,0.65$, and $0.7 M_{\odot}$. (b) We plot three $V$ model light curves of photospheric ("BB"), free-free ("FF"), and total flux ("TOTAL) for the $0.6 M_{\odot}$ WD. The photospheric emission significantly contributes to the total $V$ flux in contrast to the fast novae GK Per and V603 Aql.

To summarize, our model light curves of total $V$ flux reasonably reproduce the absolute brightnesses of optical light curves of novae with known distances, at least, in the early phase before the nebular phase or dust blackout starts. For slower novae, photospheric emission dominates the spectrum in $V$ band. For faster novae, on the other hand, free-free emission dominates the spectrum in $V$ band and therefore fast novae follow the universal decline law.

\subsection{Do Slow Novae Follow the MMRD Relation?}

In this subsection we discuss whether or not slow novae follow the MMRD relation. Theoretical free-free emission light curves of novae clearly shows a trend that a more massive WD has a brighter maximum magnitude ( $\left.\operatorname{smaller} M_{V, \max }\right)$ and a faster decline rate (smaller $t_{3}$ time). The relation between $t_{3}$ and $M_{V, \max }$ for novae is called "Maximum Magnitude vs. Rate of Decline" (MMRD) relation.

Figure 31 shows observed data points of $\left(t_{3}, M_{V, \max }\right)$ for 


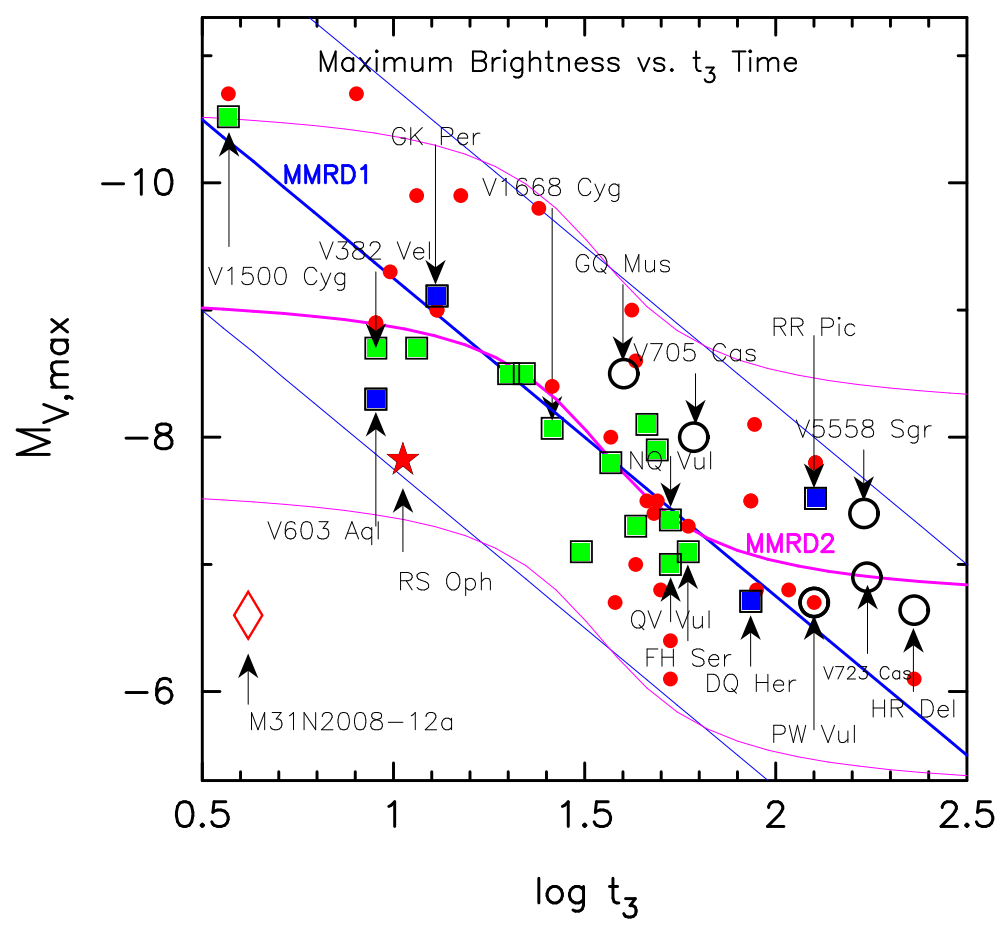

Figure 31. Maximum magnitude vs. rate of decline (MMRD) relation. Large open black circles: six novae studied in the present work, PW Vul, V705 Cas, GQ Mus, V5558 Sgr, HR Del, and V723 Cas. Red filled circles: individual novae taken from Table 5 of Downes \& Duerbeck (2000). Blue filled squares: four novae calibrated with the annual parallax method, taken from Harrison et al. (2013). Green filled squares: novae calibrated with the time-stretching method, taken from Hachisu \& Kato (2014). Blue solid line labeled "MMRD1:" Kaler-Schmidt law (Schmidt 1957) and its \pm 1.5 mag lines (see Equation (A13)). Magenta solid line labeled "MMRD2:" Della Valle \& Livio's law (della Valle \& Livio 1995) and its \pm 1.5 mag lines (see Equation (A14)). Red filled star: the recurrent nova RS Oph as an example for a very high mass accretion rate and very short timescale $t_{3}$ (Hachisu \& Kato 2014). Red open diamond: the 1 yr recurrence period M31 nova, M31N2008-12a, taken from Tang et al. (2014), i.e., $M_{g \text {, max }}=-6.6$ (maximum in $g$-band) and $t_{3, g} \approx 4.2$ days (measured in the $g$-band light curve). See text for more details.

many classical/recurrent novae. A blue solid line flanking with \pm 1.5 mag lines indicates the relation of "KalerSchmidt's law" (labeled "MMRD1," see Schmidt 1957), which is Equation (A13). A magenta solid line flanking with \pm 1.5 mag lines indicates the relation of "della Valle-Livio's law" (labeled "MMRD2," see della Valle \& Livio 1995), which is Equation (A14). Red filled circles are novae taken from Downes \& Duerbeck (2000), the distances of which were mainly derived from the nebular expansion parallax method. We show the six novae studied in the present work by large black open circles, i.e., PW Vul, V705 Cas, GQ Mus, V5558 Sgr, HR Del, and V723 Cas. Green filled squares are novae taken from Hachisu \& Kato (2010), the distance moduli of which are based on the time-stretching method. Blue filled squares indicate the four novae, V603 Aql, DQ Her, GK Per, and RR Pic, the distances of which were determined by Harrison et al. (2013) with HST annual parallaxes.

Hachisu \& Kato (2006) found that nova light curves follow a universal decline law when free-free emission dominates the continuum spectrum in optical and NIR regions. Using this property, Hachisu \& Kato (2010) found that, if two nova light curves overlap each other after one of the two is squeezed/stretched by a factor of $f_{s}\left(t^{\prime}=t / f_{s}\right)$ in the time direction, the brightnesses of the two novae obey the relation of $m_{V}^{\prime}=m_{V}-2.5 \log f_{s}$, which is the same as Equation (A6). Based on this property, they derived a MMRD relation of $M_{V, \max }=2.5 \log t_{3}-11.6$ for the chemical composition of "CO nova 2," i.e., Equation (35) in Hachisu \& Kato (2010). We can again derive a similar trend, $M_{V, \text { max }}=2.5 \log t_{3}-11.65$, i.e., Equation (A12), for another chemical composition, "CO nova 4," in Appendix A. Note that these two relations are in good agreement with Kaler-Schmidt's law. The main trend of the MMRD relation is governed by the WD mass: the more massive a WD is, the steeper the decline of a nova light curve is. Hachisu \& Kato (2010) further showed that the maximum brightness of a nova also depends on the initial envelope mass (ignition mass). This initial envelope mass depends on the mass-accretion rate to the WD (see, e.g., Kato et al. 2014, for a recent result). Hachisu \& Kato (2010) concluded that the scatter of individual MMRD points is due to various massaccretion rates to the WD even for the same WD mass. The brighter the nova is, the smaller the mass accretion rate to the WD is (see Figure 15 of Hachisu \& Kato 2010).

Harrison et al. (2013) concluded that DQ Her and GK Per almost follow the MMRD relation (MMRD1) but V603 Aql and RR Pic do not (see Figure 31). We examine the reason for about 1 mag faintness of V603 Aql compared with the blue solid line (MMRD1 relation). Hachisu \& Kato (2014) analyzed the light curves of V1500 Cyg and V603 Aql and concluded that both of these novae harbor $\mathrm{a} \sim 1.2 \mathrm{M} \odot$ WD for the envelope chemical composition of "Ne nova 2." We estimated the initial envelope masses for these two novae, that is, $M_{\text {env }}=0.92 \times 10^{-5} M_{\odot}$ for the $1.2 M_{\odot}$ WD model of V1500 Cyg and $M_{\text {env }}=0.47 \times 10^{-5} M_{\odot}$ for V603 Aql. The initial envelope mass corresponds to the envelope mass at optical maximum (see Figure 29). Thus, the difference in the ignition masses makes the apparent difference in the start time of the outbursts and in the peak brightness as shown in Figure 29. This is the main source for scatter of individual MMRD points around the proposed MMRD relation. Therefore, a smaller initial envelope mass is the main reason that V603 Aql is fainter by $\sim 1.2 \mathrm{mag}$ than the blue solid line of 
MMRD1.

Now we first examine the case of PW Vul among the seven novae studied in the present work. The MMRD point of PW Vul locates slightly ( 0.2 mag) above Kaler-Schmidt's law (MMRD1), a blue thick solid line in Figure 31. Remember that our free-free emission model light curves usually follow the averaged MMRD relation of MMRD1 (Kaler \& Schmidt's law). Thus, the agreement of PW Vul with the MMRD1 relation indicates that the initial envelope mass of PW Vul was a typical one for $\sim 0.83 M_{\odot}$ WDs and also that free-free emission dominates the spectrum in $V$ band (see Figure 10).

The next is V705 Cas. This MMRD point is much $(\sim 0.8$ mag) brighter than both of the MMRD1 and MMRD2 relations. The photospheric emission is not as much to make the $t_{3}$ time longer by a factor of 2.0 (because $2.5 \Delta \log t_{3}=$ $2.5 \log 2.0 \approx 2.5 \times 0.3 \approx 0.8$ ), as clearly shown in Figure 16 Therefore, we attribute the difference in the maximum brightness to the difference in the initial envelope mass. To confirm this, we compared the rise time of UV $1455 \AA$ flux between PW Vul and V705 Cas in Figures 10 and 16 . The UV $1455 \AA$ flux had already risen at the optical maximum in PW Vul while it had not yet in V705 Cas. This can be seen more easily in Figure 13 . The longer time before UV $1455 \AA$ peak means that the envelope mass is more massive in V705 Cas than in PW Vul. The more massive initial envelope mass makes the brighter optical maximum of V705 Cas. Thus, it locates above the blue solid line of MMRD1 relation.

The third object is GQ Mus. The MMRD point of this object is also much ( $\sim 0.8 \mathrm{mag})$ above both the MMRD1 (blue solid line) and MMRD2 (magenta solid line). GQ Mus shows a bump of $\sim 1$ mag brighter than the model light curve in the very early phase. This feature is very similar to that of V1500 Cyg. One could suppose that this bump is the origin of the deviation from the blue solid line of MMRD1 relation, but V1500 Cyg does not show such a large deviation (see Figure 31). Therefore, we suppose that photospheric emission is the main source of the deviation. We found, from Figure $17 \mathrm{a}$ ), that the $t_{3}$ time of the total flux (black solid line) is 2.3 times longer compared with the case of free-free only (blue solid line). This effect makes the $t_{3}$ time longer by about $\Delta \log t_{3}=\log 2.3 \approx 0.35$. This corresponds to the increase in the brightness by $2.5 \Delta \log t_{3}=2.5 \times 0.35=0.9$ mag in the MMRD diagram, being roughly consistent with the present position of GQ Mus in Figure 31. Thus, we conclude that GQ Mus is above the MMRD relation because of photospheric emission effect.

The remaining objects are static-to-wind transition novae, RR Pic, V5558 Sgr, HR Del, and V723 Cas. These MMRD points are also much (0.8-1.2 mag) above the blue solid line of MMRD1 but HR Del and V723 Cas are consistent with the MMRD2. From Figure 26 of V723 Cas, we see that the $t_{3}$ time estimated along our total $V$ flux model (black solid line) is $\sim 2.2$ times longer than the case of free-free only (blue solid line). Note again that our free-free emission model light curves usually follow the averaged MMRD relation of MMRD1 (Kaler \& Schmidt's law). The photospheric emission effect raises the brightness by $2.5 \Delta \log t_{3}=2.5 \log 2.2 \approx$ 0.8 mag compared with the MMRD1 (blue solid line), being roughly consistent with the position of V723 Cas in Figure 31. Similarly for HR Del, we obtain, from Figure 25 (a), a factor of $\sim 2.2$ and a raise of $2.5 \Delta \log t_{3}=2.5 \log 2.2 \approx 0.8 \mathrm{mag}$ owing to the photospheric emission effect. This is also consistent with the position of HR Del in Figure 31. V5558 Sgr and RR Pic showed much brighter ( $\sim 1.2 \mathrm{mag})$ optical maxima than the MMRD1 (blue solid line). These two novae showed prominent amplitudes of oscillations during the multiple-peak. We think that these large peaks are related to more massive envelopes compared with those of HR Del and V723 Cas. In fact, the amplitude of multiple-peak is decreasing in V5558 Sgr, suggesting reduction of the envelope mass due to mass loss. The same explanation is possible in RR Pic, whose MMRD point is also 1.2 mag brighter than the MMRD1 relation (blue solid line).

It is interesting to see the position of the recurrent nova RS Oph (red filled star) and the 1 yr recurrence period M31 nova, M31N2008-12a (red open diamond) in Figure 31. RS Oph locates $1.2 \mathrm{mag}$ below the MMRD1. This faintness corresponds to a much smaller envelope mass at optical maximum, suggesting a massive WD and very high mass accretion rate. This situation is very consistent with the total picture of recurrent novae; a very massive WD close to the Chandrasekhar mass and a high mass accretion rate to the WD (e.g., Hachisu \& Kato 2001). In this figure, we adopt $M_{V, \max }=-7.8$ and $t_{3}=10.5$ days with the distance of $d=1.4 \mathrm{kpc}$ (Hachisu et al. 2006; Barry et al. 2006; Hachisu \& Kato 2014), absorption of $A_{V}=3.1 E(B-$ $V)=3.1 \times 0.65=2.0$ (Hachisu \& Kato 2014), $m_{V, \max }=5.0$ (Rosino \& Iijima 1987), and $t_{3}=10.5$ days from optical light curve fitting with our free-free model light curves (Hachisu \& Kato 2001; Hachisu et al. 2006, 2007). The 1 yr recurrence period M31 nova, M31N2008-12a is depicted by a red open diamond. It is very faint, i.e., $M_{g, \max }=-6.6$ (maximum in $g$-band) and $t_{3, g} \approx 4.2$ days (measured in the $g$-band light curve), taken from Tang et al. (2014). The 1 yr recurrence period is close to the shortest recurrence period of novae, suggesting a very massive WD close to the Chandrasekhar mass and a very high accretion rate (see, e.g., Tang et al. 2014; Kato et al. 2014), thus a very small envelope mass. These support our conclusion that the peak brightness of a nova depends on the initial envelope mass as well as the WD mass itself.

To summarize, the primary parameter of the MMRD relation is the WD mass and the secondary parameter is the initial envelope mass. Variations in the initial envelope mass is the origin of scatter from the averaged MMRD relation, MMRD1. More massive envelopes correspond to the region above the MMRD1 line and less massive envelopes correspond to the region below the MMRD1 line. Photospheric emission is the third factor of the MMRD relation but becomes more important in slow novae, because it makes $t_{3}$ time longer in low mass WDs.

\section{CONCLUSIONS}

There have been suggested several scaling laws for classical nova light curves (e.g., Hachisu et al. 2008, for a summary). Hachisu \& Kato (2006) proposed that classical nova light curves follow a universal shape when continuum flux is dominated by free-free emission. Using this property, Hachisu \& Kato (2010) theoretically explained the main trend of the MMRD relations. These results were confirmed only for fast novae. In this paper, we examined seven novae of slow evolution, in which photospheric emission could considerably contribute to the continuum spectra in $V$ band rather than free-free emission. We obtain the following main results: 1. Based on various observational estimates in literature, we estimated physical parameters of the slow nova PW Vul. We adopted the distance modulus of $(m-M)_{V}=13.0$ in $V$ band, 
Table 4

Light Curves of CO Novae

\begin{tabular}{|c|c|c|c|c|c|c|c|c|c|c|c|c|c|c|}
\hline $\begin{array}{c}m_{\mathrm{ff}} \\
(\mathrm{mag})\end{array}$ & $\begin{array}{c}0.55 M_{\odot} \\
\quad \text { (day) }\end{array}$ & $\begin{array}{c}0.6 M_{\odot} \\
\text { (day) }\end{array}$ & $\begin{array}{c}0.65 M_{\odot} \\
\quad \text { (day) }\end{array}$ & $\begin{array}{c}0.7 M_{\odot} \\
\text { (day) }\end{array}$ & $\begin{array}{c}0.75 M_{\odot} \\
\quad \text { (day) }\end{array}$ & $\begin{array}{c}0.8 M_{\odot} \\
\text { (day) }\end{array}$ & $\begin{array}{c}0.85 M_{\odot} \\
\quad \text { (day) }\end{array}$ & $\begin{array}{c}0.9 M_{\odot} \\
\text { (day) }\end{array}$ & $\begin{array}{c}0.95 M_{\odot} \\
\quad \text { (day) }\end{array}$ & $\begin{array}{c}1.0 M_{\odot} \\
\text { (day) }\end{array}$ & $\begin{array}{c}1.05 M_{\odot} \\
\quad \text { (day) }\end{array}$ & $\begin{array}{c}1.1 M_{\odot} \\
\text { (day) }\end{array}$ & $\begin{array}{c}1.15 M_{\odot} \\
\quad \text { (day) }\end{array}$ & $\begin{array}{c}1.2 M_{\odot} \\
\text { (day) }\end{array}$ \\
\hline 3.000 & 0.0 & 0.0 & 0.0 & 0.0 & 0.0 & 0.0 & 0.0 & 0.0 & 0.0 & 0.0 & 0.0 & 0.0 & 0.0 & 0.0 \\
\hline 3.250 & 3.429 & 2.630 & 2.591 & 2.210 & 2.090 & 1.400 & 1.153 & 1.060 & 0.960 & 0.859 & 0.761 & 0.689 & 0.621 & 0.566 \\
\hline 3.500 & 9.489 & 7.150 & 5.251 & 4.480 & 4.200 & 2.810 & 2.399 & 2.130 & 1.920 & 1.735 & 1.505 & 1.372 & 1.244 & 1.125 \\
\hline 3.750 & 16.73 & 11.92 & 10.28 & 6.990 & 6.360 & 4.500 & 3.686 & 3.220 & 2.890 & 2.605 & 2.263 & 2.033 & 1.845 & 1.685 \\
\hline 4.000 & 25.84 & 18.62 & 15.93 & 10.22 & 8.600 & 6.560 & 5.106 & 4.370 & 3.890 & 3.485 & 3.035 & 2.706 & 2.449 & 2.243 \\
\hline 4.250 & 35.20 & 26.12 & 21.82 & 14.76 & 11.43 & 8.680 & 6.586 & 5.580 & 5.010 & 4.375 & 3.822 & 3.392 & 3.071 & 2.811 \\
\hline 4.500 & 44.97 & 33.92 & 27.89 & 20.55 & 15.39 & 10.84 & 8.296 & 6.830 & 6.200 & 5.345 & 4.625 & 4.102 & 3.732 & 3.433 \\
\hline 4.750 & 56.92 & 41.91 & 34.15 & 26.12 & 19.57 & 13.21 & 10.14 & 8.120 & 7.440 & 6.545 & 5.574 & 4.831 & 4.411 & 4.082 \\
\hline 5.000 & 73.18 & 53.71 & 41.54 & 32.00 & 23.94 & 16.31 & 12.77 & 10.04 & 8.960 & 7.785 & 6.754 & 5.783 & 5.216 & 4.770 \\
\hline 5.250 & 93.75 & 67.24 & 51.78 & 38.17 & 28.92 & 19.55 & 15.39 & 12.16 & 10.73 & 9.085 & 7.984 & 6.923 & 6.235 & 5.656 \\
\hline 5.500 & 117.3 & 82.44 & 62.96 & 44.79 & 34.27 & 23.01 & 17.83 & 14.32 & 12.62 & 10.44 & 8.994 & 7.933 & 7.155 & 6.536 \\
\hline 5.750 & 143.2 & 100.6 & 76.05 & 54.33 & 41.01 & 27.27 & 20.39 & 16.29 & 14.36 & 11.90 & 10.06 & 8.753 & 7.828 & 7.115 \\
\hline 6.000 & 169.1 & 119.9 & 90.21 & 64.79 & 48.34 & 31.96 & 23.29 & 18.37 & 16.22 & 13.43 & 11.21 & 9.623 & 8.538 & 7.732 \\
\hline 6.250 & 196.2 & 138.8 & 105.5 & 76.07 & 56.51 & 37.00 & 27.03 & 20.66 & 18.27 & 15.09 & 12.53 & 10.61 & 9.349 & 8.392 \\
\hline 6.500 & 225.9 & 159.4 & 122.1 & 88.03 & 65.54 & 42.74 & 31.12 & 23.62 & 20.77 & 16.85 & 13.94 & 11.71 & 10.28 & 9.142 \\
\hline 6.750 & 259.4 & 181.6 & 138.6 & 100.2 & 75.15 & 49.14 & 35.57 & 26.89 & 23.51 & 18.93 & 15.51 & 12.90 & 11.30 & 9.962 \\
\hline 7.000 & 297.1 & 205.6 & 156.3 & 113.5 & 84.87 & 56.14 & 40.49 & 30.41 & 26.51 & 21.22 & 17.29 & 14.26 & 12.36 & 10.80 \\
\hline 7.250 & 340.5 & 232.5 & 175.9 & 127.7 & 95.18 & 63.63 & 45.86 & 34.25 & 29.98 & 23.67 & 19.22 & 15.75 & 13.54 & 11.71 \\
\hline 7.500 & 393.1 & 264.4 & 199.4 & 143.1 & 106.3 & 71.08 & 51.74 & 38.45 & 33.37 & 26.21 & 21.15 & 17.30 & 14.76 & 12.70 \\
\hline 7.750 & 455.2 & 300.8 & 225.9 & 160.7 & 120.4 & 79.16 & 57.70 & 42.99 & 36.91 & 29.00 & 23.22 & 18.82 & 15.97 & 13.63 \\
\hline 8.000 & 519.0 & 345.8 & 255.8 & 182.1 & 135.9 & 88.73 & 64.06 & 47.67 & 40.84 & 32.03 & 25.48 & 20.47 & 17.28 & 14.64 \\
\hline 8.250 & 592.2 & 394.2 & 290.9 & 206.4 & 153.7 & 99.40 & 71.42 & 52.84 & 45.17 & 35.39 & 27.97 & 22.27 & 18.72 & 15.75 \\
\hline 8.500 & 656.8 & 444.5 & 331.9 & 235.5 & 175.4 & 112.0 & 80.12 & 58.82 & 50.36 & 39.11 & 30.89 & 24.42 & 20.40 & 17.03 \\
\hline 8.750 & 730.1 & 499.8 & 370.0 & 267.3 & 199.2 & 127.4 & 89.99 & 65.73 & 56.68 & 43.55 & 34.11 & 26.93 & 22.36 & 18.63 \\
\hline 9.000 & 804.7 & 551.1 & 403.0 & 299.8 & 224.8 & 144.9 & 102.5 & 74.22 & 64.08 & 48.99 & 38.34 & 29.98 & 24.81 & 20.53 \\
\hline 9.250 & 866.8 & 600.1 & 440.8 & 331.6 & 253.0 & 163.6 & 116.7 & 84.49 & 73.31 & 55.38 & 43.03 & 33.63 & 27.83 & 22.92 \\
\hline 9.500 & 935.9 & 647.3 & 484.0 & 367.3 & 279.2 & 185.1 & 131.6 & 96.54 & 83.61 & 63.32 & 49.08 & 38.00 & 31.37 & 25.68 \\
\hline 9.750 & 1000. & 700.4 & 533.6 & 399.8 & 301.6 & 208.1 & 148.5 & 108.9 & 93.88 & 72.14 & 55.91 & 43.18 & 35.47 & 28.95 \\
\hline 10.00 & 1059. & 750.2 & 570.9 & 429.1 & 322.4 & 227.8 & 167.2 & 122.6 & 105.7 & 81.11 & 62.98 & 48.79 & 39.93 & 32.54 \\
\hline 10.25 & 1123. & 795.2 & 605.3 & 460.9 & 342.1 & 246.8 & 179.6 & 137.8 & 117.2 & 91.19 & 70.78 & 54.70 & 44.67 & 36.30 \\
\hline 10.50 & 1190. & 842.8 & 641.7 & 488.9 & 363.1 & 267.1 & 193.5 & 150.2 & 125.0 & 101.7 & 79.43 & 61.21 & 49.89 & 40.43 \\
\hline 10.75 & 1261. & 893.3 & 680.3 & 518.7 & 385.2 & 283.6 & 208.8 & 162.4 & 133.2 & 111.3 & 86.87 & 67.75 & 54.85 & 44.76 \\
\hline 11.00 & 1336. & 946.7 & 721.2 & 550.2 & 408.7 & 301.0 & 225.9 & 175.6 & 141.9 & 118.4 & 94.26 & 73.77 & 59.08 & 48.84 \\
\hline 11.25 & 1415. & 1003. & 764.5 & 583.5 & 433.6 & 319.5 & 244.7 & 187.1 & 151.2 & 126.0 & 100.1 & 79.34 & 63.62 & 52.65 \\
\hline 11.50 & 1500. & 1063. & 810.4 & 618.9 & 459.9 & 339.0 & 259.8 & 199.1 & 160.9 & 134.0 & 106.4 & 85.27 & 68.48 & 56.40 \\
\hline 11.75 & 1589. & 1127. & 859.0 & 656.3 & 487.8 & 359.7 & 275.6 & 211.7 & 171.3 & 142.4 & 113.0 & 90.78 & 73.68 & 60.10 \\
\hline 12.00 & 1684. & 1194. & 910.5 & 696.0 & 517.4 & 381.7 & 292.4 & 225.1 & 182.3 & 151.4 & 120.0 & 96.66 & 79.07 & 63.80 \\
\hline 12.25 & 1784. & 1265. & 965.0 & 738.0 & 548.7 & 405.0 & 310.1 & 239.3 & 193.9 & 160.9 & 127.4 & 102.6 & 83.82 & 67.73 \\
\hline 12.50 & 1890. & 1341. & 1022. & 782.5 & 581.9 & 429.6 & 329.0 & 254.3 & 206.2 & 170.9 & 135.3 & 108.9 & 88.86 & 71.88 \\
\hline 12.75 & 2002. & 1421. & 1084. & 829.7 & 617.0 & 455.7 & 348.9 & 270.2 & 219.3 & 181.6 & 143.6 & 115.5 & 94.19 & 76.28 \\
\hline 13.00 & 2122 . & 1505. & 1148. & 879.6 & 654.3 & 483.3 & 370.0 & 287.0 & 233.1 & 192.9 & 152.5 & 122.6 & 99.88 & 80.94 \\
\hline 13.25 & 2248 . & 1595. & 1217. & 932.5 & 693.7 & 512.5 & 392.4 & 304.9 & 247.7 & 204.8 & 161.8 & 130.0 & 105.8 & 85.88 \\
\hline 13.50 & 2381. & 1690. & 1290. & 988.0 & 735.4 & 543.5 & 416.0 & 323.8 & 263.2 & 217.5 & 171.7 & 137.9 & 112.2 & 91.11 \\
\hline 13.75 & 2523. & 1791. & 1367. & 1048. & 779.7 & 576.4 & 441.1 & 343.8 & 279.6 & 230.9 & 182.2 & 146.3 & 118.9 & 96.64 \\
\hline 14.00 & 2673. & 1898. & 1448. & 1111. & 826.5 & 611.2 & 467.7 & 365.0 & 297.0 & 245.1 & 193.3 & 155.2 & 126.0 & 102.5 \\
\hline 14.25 & 2832. & 2010. & 1535. & 1177. & 876.2 & 648.0 & 495.9 & 387.5 & 315.4 & 260.1 & 205.1 & 164.6 & 133.5 & 108.7 \\
\hline 14.50 & 3000. & 2130. & 1626. & 1248. & 928.8 & 687.0 & 525.7 & 411.2 & 334.9 & 276.1 & 217.5 & 174.5 & 141.5 & 115.3 \\
\hline 14.75 & 3178. & 2257. & 1723. & 1323. & 984.4 & 728.4 & 557.3 & 436.4 & 355.6 & 292.9 & 230.7 & 185.1 & 150.0 & 122.3 \\
\hline 15.00 & 3367. & 2391. & 1826. & 1402. & 1043. & 772.2 & 590.8 & 463.1 & 377.5 & 310.8 & 244.7 & 196.3 & 158.9 & 129.7 \\
\hline X-ray ${ }^{b}$ & 8210 & 6150 & 4700 & 3650 & 2640 & 1900 & 1370 & 980 & 730 & 540 & 370 & 250 & 169 & 112 \\
\hline $\log f_{\mathrm{s}}^{\mathrm{c}}$ & 0.60 & 0.47 & 0.39 & 0.29 & 0.17 & 0.06 & -0.05 & -0.15 & -0.24 & -0.33 & -0.43 & -0.55 & -0.67 & -0.77 \\
\hline$M_{\mathrm{W}}^{\mathrm{d}}$ & 5.5 & 5.1 & 4.6 & 4.2 & 3.8 & 3.3 & 2.9 & 2.5 & 2.2 & 1.8 & 1.5 & 1.2 & 0.9 & 0.7 \\
\hline
\end{tabular}

a chemical composition of the envelope is assumed to be that of "CO nova 4" in Table2

$\mathrm{b}$ duration of supersoft X-ray phase in units of days.

${ }^{\mathrm{c}}$ stretching factor against the $0.83 M_{\odot}$ model which is the best fit light curve for the PW Vul UV $1455 \AA$ observation in Figure 33

$\mathrm{d}$ absolute magnitudes at the bottom point in Figure 34 by assuming $(m-M)_{V}=13.0$ (PW Vul).

extinction of $E(B-V)=0.55$, and distance of $d=1.8 \mathrm{kpc}$ for PW Vul.

2. We divide approximately a nova spectrum into two components, one is photospheric and the other is optically thick free-free emission. During the optically thick wind phase of the slow nova PW Vul, free-free emission dominates the continuum spectrum in NIR bands while photospheric emission contributes, to some extent, to the continuum spectrum in $V$ band.

3. We calculated the total $V$ model light curves (the sum of free-free plus photospheric emission) of classical novae for the chemical composition of $X=0.55, Y=0.23, Z=0.02$, and $X_{\mathrm{CNO}}=0.20$ ("CO nova 4"), which is close to that of PW Vul. By simultaneous fitting of the total $V$ light curve model and the blackbody UV $1455 \AA$ light curve model, we determine the WD mass of PW Vul to be $\sim 0.83 M_{\odot}$.

4. Using the distance modulus of $(m-M)_{V}=13.0$ for PW Vul and properties of the universal decline law, we determined the absolute magnitudes of free-free emission light curves for various WD masses with the envelope chemical composition of "CO nova 4." Based on the universal decline law, we also derived the MMRD relations for "CO nova 4." This theoret- 
ical MMRD relations are consistent with the ever proposed empirical formulae (Appendix A].

5. We also analyzed the moderately-fast nova V705 Cas and estimated the WD mass to be $\sim 0.78 M_{\odot}$. Even for this WD mass, we found that free-free emission still dominates the continuum spectrum in $V$ and NIR bands. We obtained the distance modulus of $(m-M)_{V}=13.4$ and the color excess of $E(B-V)=0.45$, which are consistent with those obtained from other observations.

6. We reanalyzed the fast nova GQ Mus. Fitting our model light curves with optical $V, \mathrm{UV} 1455 \AA$, and supersoft X-ray light curve observations, we confirmed that the WD mass is $\sim 0.65 M_{\odot}$ for an assumed chemical composition of $X=0.35$, $Y=0.33, Z=0.02$, and $X_{\mathrm{CNO}}=0.30$ ("CO nova 2"). For this low WD mass, we found that photospheric emission is more important and dominates the continuum spectrum in $V$ band. We consistently obtained the distance modulus of $(m-M)_{V}=$ 15.7 , color excess of $E(B-V)=0.45$, and distance of $d=$ $7.3 \mathrm{kpc}$.

7. We further analyzed four very slow novae, RR Pic, V5558 Sgr, HR Del, and V723 Cas, and estimated their WD masses as low as $\sim 0.5-0.55 M_{\odot}$. We also consistently obtained the distance moduli, color excesses, and distances of V5558 Sgr, HR Del, and V723 Cas as $(m-M)_{V}=13.9,10.4$, and 14.0, $E(B-V)=0.7,0.15$, and 0.35 , and $d=2.2,0.97$, and $3.9 \mathrm{kpc}$, respectively. We found that, in optical $V$ band, photospheric emission is more important than free-free emission in these four novae.

8. We confirmed that our total $V$ flux model light curves reasonably reproduce the absolute brightnesses of four novae with known distances, i.e., RR Pic, GK Per, V603 Aql, and DQ Her. We found that free-free emission dominates the spectra in $V$ band for the fast novae GK Per and V603 Aql but photospheric emission significantly contributes to the total $V$ flux for the slow novae RR Pic and DQ Her.

9. The four very slow novae, RR Pic, V5558 Sgr, HR Del, and V723 Cas lie about $0.8-1.3$ mag above Kaler-Schmidt's MMRD relation. In these novae, photospheric emission dominates the continuum spectra in $V$ band and makes their $t_{3}$ times much longer $(\sim 2$ times) than that of free-free emission only. Because the model light curves of free-free emission follow Kaler-Schmidt's MMRD relation, the total (free-free plus photospheric) flux light curves raise the MMRD brightness by $2.5 \log 2 \approx 0.8 \mathrm{mag}$. This is the reason that the MMRD points of these novae are about 1 mag brighter than KalerSchmidt's MMRD relation.

We are grateful to Angelo Cassatella for fruitful discussion and critical reading of the manuscript. We also thank the American Association of Variable Star Observers (AAVSO) and Variable Star Observers League of Japan (VSOLJ) for the archival data of PW Vul, V705 Cas, GQ Mus, RR Pic, V5558 Sgr, HR Del, and V723 Cas. This research has been supported in part by the Grant-in-Aid for Scientific Research (22540254, 24540227) of the Japan Society for the Promotion of Science.

\section{APPENDIX}

\section{A. ABSOLUTE MAGNITUDES OF FREE-FREE MODEL LIGHT CURVES}

We have already calibrated the absolute magnitude of the $0.83 M_{\odot}$ free-free model light curve for "CO nova 4 " in Section 3.4 which is defined by $M_{\mathrm{w}}=3.0$ at the end point of the free-free emission model light curve. In this appendix, we calibrate all free-free model light curves for various WD masses using $M_{\mathrm{w}}=3.0$ of the $0.83 M_{\odot}$ WD model, i.e., the PW Vul data. In other words, we will determine the absolute magnitudes, $M_{\mathrm{w}}$, for all WD mass light curves in Figure 32.

\section{A.1. Model light curves of free-free emission}

In terms of free-free emission, Hachisu \& Kato (2006) obtained model light curves of novae in $0.05 M_{\odot}$ steps for masses in the range $M_{\mathrm{WD}}=0.55-1.2 M_{\odot}$ with the chemical composition "CO nova 4." The flux is calculated from

$$
F_{\nu}^{\left\{M_{\mathrm{wD}}\right\}}(t)=C\left[\frac{\dot{M}_{\mathrm{wind}}^{2}}{v_{\mathrm{ph}}^{2} R_{\mathrm{ph}}}\right]_{(t)}^{\left\{M_{\mathrm{WD}}\right\}},
$$

where $\dot{M}_{\text {wind }}$ is the wind mass-loss rate, $v_{\mathrm{ph}}$ is the wind velocity at the photosphere, $R_{\mathrm{ph}}$ is the photospheric radius of each wind solution, and $C$ is the proportionality constant (see Equation (9) of Hachisu \& Kato 2006). Note that the flux $F_{\nu}$ is independent of the frequency $\nu$ in the case of optically-thin free-free emission. The details of calculations are presented in Hachisu \& Kato (2006, 2010). Then the magnitude of model light curves are calculated as

$$
m_{\mathrm{ff}}=-2.5 \log \left[\frac{\dot{M}_{\mathrm{wind}}^{2}}{v_{\mathrm{ph}}^{2} R_{\mathrm{ph}}}\right]_{(t)}^{\left\{M_{\mathrm{WD}}\right\}}+G^{\left\{M_{\mathrm{WD}}\right\}} .
$$

The numerical data entering in Equation (A2) are tabulated in Table 4. Subscript $(t)$ denotes the time dependence, while superscript $\left\{M_{\mathrm{WD}}\right\}$ indicates a model parameter. The last row (15th mag) of each column in Table 4 corresponds to the end of the wind phase in each light curve sequence. In other words, we define the constant $G^{\left\{M_{\mathrm{WD}}\right\}}$ in Equation (A2) such that the last (lowest) point of each light curve (the end of an optically thick wind phase) is 15 th mag. This helps to shorten the table. The magnitudes of free-free emission $m_{\mathrm{ff}}$ are plotted in Figure 32

\section{A.2. Time-normalized light curves}

The free-free emission model light curves in Figure 32 have a very similar shape. For the chemical compositions of "CO nova 2" and "Ne nova 2," Hachisu \& Kato (2010) showed that model light curves corresponding to different masses have a homologous behaviour, in the sense that they overlap each other if properly squeezed or stretched along time. Here we show that the same property applies to the case of chemical composition "CO nova 4." Figure 33 demonstrates that the free-free emission model light curves in Figure 32 overlap each other if they are properly squeezed/stretched along time.

The time-scaling factor, $f_{\mathrm{s}}$, of each model was determined by increasing or decreasing $f_{\mathrm{s}}$ until the model UV $1455 \AA$ light curve shape matches the observational points. We also normalize the peak flux of our model UV $1455 \AA$ light curve to match the observational peak. For example, the evolution of the $0.8 M_{\odot}$ model evolves 1.15 times slower than the PW Vul observation, so $f_{\mathrm{s}} \approx 1.15$. The $0.85 M_{\odot}$ model evolves 1.1 times faster than that, so $f_{\mathrm{s}} \approx 0.9$. The scaling factor $\log f_{s}$ thus obtained are tabulated in Table 4.

If we squeeze the timescale of our model light curves with $t^{\prime}=t / f_{s}$ as shown in Figure 33 , these light curves are written 


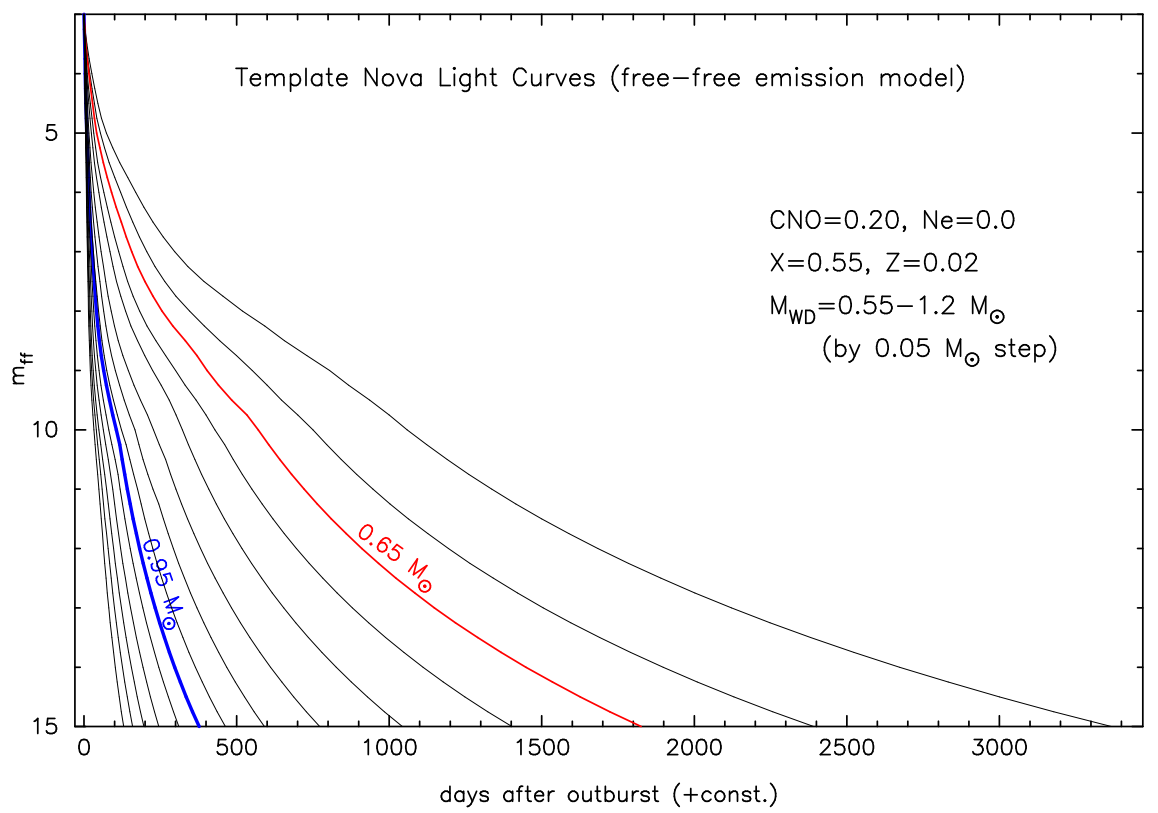

Figure 32. Magnitudes of our free-free emission model light curves for WD masses of $0.55-1.2 M_{\odot}$ in $0.05 M_{\odot}$ steps, numerical data of which are tabulated in Table 4 We adopt the chemical composition "CO nova 4" in Table 2 for these WD envelopes. The decay timescale depends on the WD mass. Two light curves are highlighted by a red thick solid line $\left(0.65 M_{\odot}\right)$ and a blue thick solid line $\left(0.95 M_{\odot}\right)$.

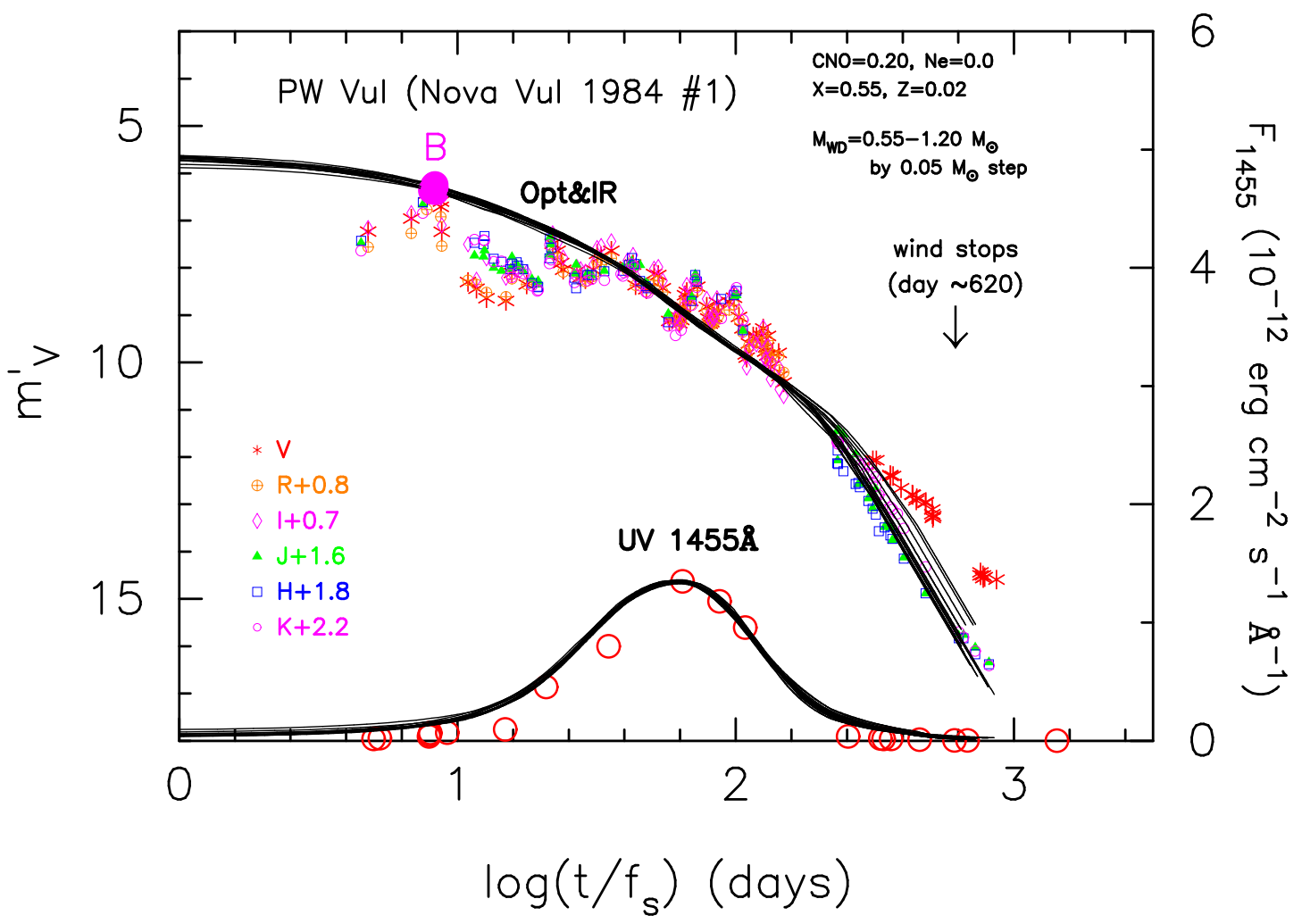

Figure 33. Same as Figure 8 but all the model light curves of free-free emission and UV $1455 \AA$ blackbody emission for various WD masses, which are rescaled to overlap each other. Each timescaling factor of $f_{\mathrm{s}}$ is tabulated in Table 4 The right edge of each free-free emission model light curve corresponds to the epoch when the optically thick winds stop. The end epoch of winds, day $\sim 620$ for the $0.83 M_{\odot}$ WD, is denoted by an arrow. Point B corresponds to the peak of $V$ magnitude of the PW Vul outburst. Here, we assume the start of the day $(t=0)$ as JD 2445910.0 for the observational points of PW Vul. 


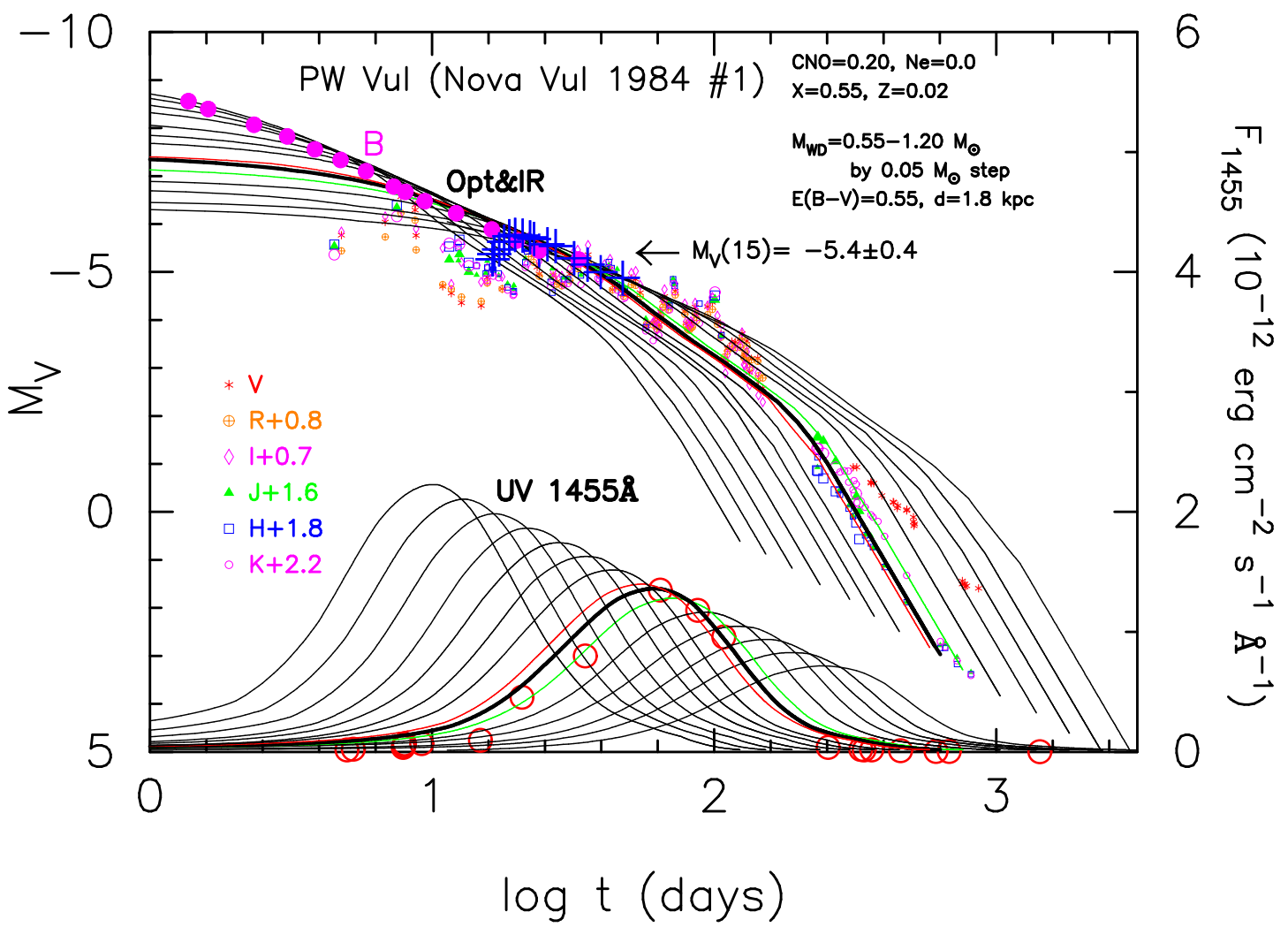

Figure 34. Same as Figure 33 but for absolute magnitudes and real timescales. We have calibrated the free-free model light curves by a distance modulus of $(m-M)_{V}=13.0$ for PW Vul and restored the absolute magnitude of each free-free emission light curve (labeled "OPT\&IR") by Equations A8 and ( $m-$ $M)_{V, \mathrm{PW} \text { Vul }}=13.0$. The position at point B in Figure 33 is indicated by a magenta filled circle on each light curve. We also show the magnitude, $M_{V}(15), 15$ days after the optical maximum, by a blue cross on each light curve. We obtain an average value of $M_{V}(15)=-5.4 \pm 0.4$ among $0.7-1.05 M_{\odot}$ WDs. UV $1455 \AA$ model light curves are also restored in the real timescale and flux at the distance of $10 \mathrm{kpc}$ without absorption. Black thick solid lines denote those for the $0.83 M_{\odot}$ WD model, red thin solid ones do for the $0.85 M_{\odot}$, and green thin solid ones do for the $0.8 M_{\odot}$ WD. 


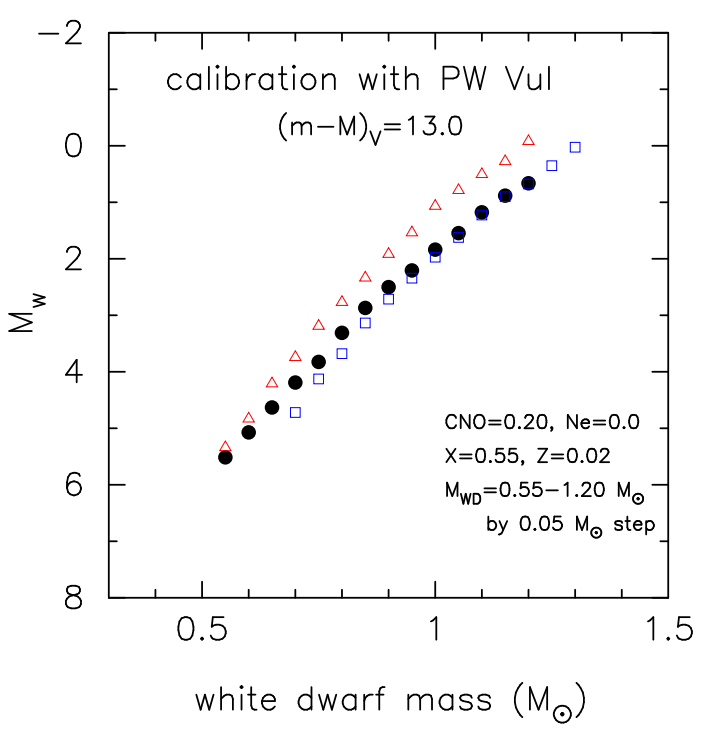

Figure 35. The absolute magnitude at the end point of each free-free emission model light curve, $M_{\mathrm{w}}$, is plotted against the WD mass for the chemical composition of "CO nova 4" (filled circles). We also added two other chemical composition models of "CO nova 2" (open triangles) and "Ne nova 2" (open squares) taken from Hachisu \& Kato (2010). See text for more details.

as

$$
m_{V}^{\prime\left\{M_{\mathrm{WD}}\right\}}\left(t^{\prime}\right)=-2.5 \log \left[\frac{\dot{M}_{\mathrm{wind}}^{2}}{v_{\mathrm{ph}}^{2} R_{\mathrm{ph}}}\right]_{\left(t^{\prime} f_{s}\right)}^{\left\{M_{\mathrm{WD}}\right\}}+K_{V},
$$

where $K_{V}$ is a constant common for all WD masses. Because they all overlap each other (i.e., the universal decline law), we regard that all these are the same phenomena. Then, it indicates that

$$
\left[\frac{\dot{M}_{\mathrm{wind}}^{2}}{v_{\mathrm{ph}}^{2} R_{\mathrm{ph}}}\right]_{\left(t^{\prime} f_{s}\right)}^{\left\{M_{\mathrm{wD}}\right\}}=\left[\frac{\dot{M}_{\mathrm{wind}}^{2}}{v_{\mathrm{ph}}^{2} R_{\mathrm{ph}}}\right]_{\left(t^{\prime} f_{s}\right)}^{\left\{0.83 M_{\odot}\right\}}=\left[\frac{\dot{M}_{\mathrm{wind}}^{2}}{v_{\mathrm{ph}}^{2} R_{\mathrm{ph}}}\right]_{\left(t^{\prime}\right)}^{\left\{0.83 M_{\odot}\right\}},
$$

for all $M_{\mathrm{WD}}$. Note that $f_{s}=1$ for the $0.83 M_{\odot}$ WD.

In general, if we squeeze the timescale of a physical phenomenon by a factor of $f_{\mathrm{s}}$ (i.e., $t^{\prime}=t / f_{\mathrm{s}}$ ), we covert the frequency to $\nu^{\prime}=f_{\mathrm{s}} \nu$ and the flux of free-free emission to $F_{\nu^{\prime}}^{\prime}=f_{\mathrm{s}} F_{\nu}$ because

$$
\frac{d}{d t^{\prime}}=f_{\mathrm{s}} \frac{d}{d t} .
$$

Substituting $F_{\nu^{\prime}}^{\prime}=F_{\nu}^{\prime}$ (independent of the frequency in optically-thin free-free emission) into $F_{\nu^{\prime}}^{\prime}=f_{\mathrm{s}} F_{\nu}$ and integrating $F_{\nu}^{\prime}=f_{\mathrm{s}} F_{\nu}$ with the $V$-filter response function, we have the following relation, i.e.,

$$
m_{V}^{\prime}\left(t / f_{s}\right)=m_{V}(t)-2.5 \log f_{\mathrm{s}}
$$

Substituting Equation (A4) into (A3), and then Equation A3 into (A6), we obtain the apparent $V$ magnitudes of

$$
m_{V}^{\left\{M_{\mathrm{WD}}\right\}}(t)=2.5 \log f_{\mathrm{s}}-2.5 \log \left[\frac{\dot{M}_{\mathrm{wind}}^{2}}{v_{\mathrm{ph}}^{2} R_{\mathrm{ph}}}\right]_{\left(t / f_{s}\right)}^{\left\{0.83 M_{\odot}\right\}}+K_{V},
$$

where note that $f_{s}$ is the time-scaling factor for the WD with mass of $M_{\mathrm{WD}}$, not for the $0.83 M_{\odot} \mathrm{WD}$.

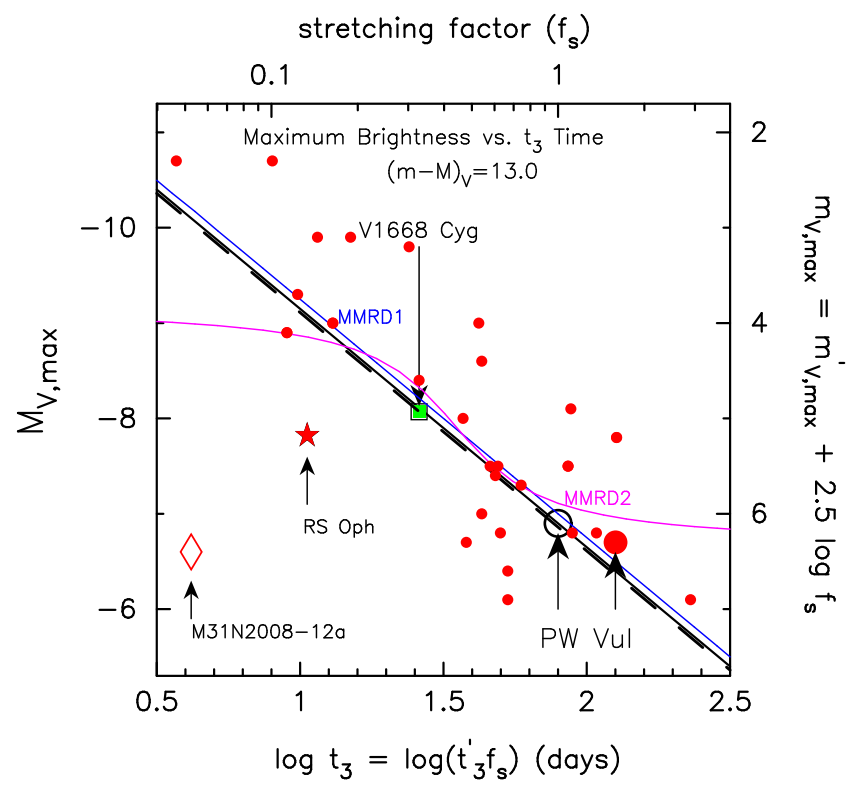

Figure 36. Maximum magnitude vs. rate of decline (MMRD) relation. Black solid line, i.e., Equation A12: our model MMRD relation calibrated with PW Vul (large black open circle). Black dashed line: another model MMRD relation calibrated with V1668 Cyg (green filled square, Hachisu \& Kato 2010), i.e., $M_{V \text {, } \max }=2.5 \log t_{3}-11.6$. Blue solid line labeled "MMRD1," i.e., Equation (A13): Kaler-Schmidt law (Schmidt 1957). Magenta solid line labeled "MMRD2," i.e., Equation (A14): della Valle \& Livio's law (della Valle \& Livio 1995). Red filled star: the recurrent nova RS Oph as an example for a very high mass accretion rate and very short timescale $t_{3}$ (very small $f_{\mathrm{s}}$ ) (Hachisu \& Kato 2010). Red open diamond: the $1 \mathrm{yr}$ recurrence period M31 nova, M31N2008-12a, taken from Tang et al. (2014), i.e., $M_{g, \max }=-6.6$ (maximum in $g$-band) and $t_{3} \approx 4.2$ days (measured in the $g$-band light curve). Red filled circles: individual novae taken from Table 5 of Downes \& Duerbeck (2000). Large red filed circle: PW Vul taken from Table 5 of Downes \& Duerbeck (2000). Large open black circle: PW Vul estimated from our model light curve of $0.83 M_{\odot}$ WD. See text for more details.

\section{A.3. Absolute magnitudes of nova light curves}

The corresponding absolute magnitudes of the light curves can be readily obtained from Equation (A7) and from the distance modulus of PW Vul, i.e.,

$$
\begin{aligned}
M_{V}^{\left\{M_{\mathrm{WD}}\right\}}(t)= & m_{V}^{\left\{M_{\mathrm{WD}}\right\}}(t)-(m-M)_{V, \mathrm{PW} \text { Vul }} \\
= & 2.5 \log f_{\mathrm{s}}-2.5 \log \left[\frac{\dot{M}_{\mathrm{wind}}^{2}}{v_{\mathrm{ph}}^{2} R_{\mathrm{ph}}}\right]_{\left(t / f_{s}\right)}^{\left\{0.83 M_{\odot}\right\}} \\
& +K_{V}-(m-M)_{V, \mathrm{PW} \mathrm{Vul}} \\
= & 2.5 \log f_{\mathrm{s}}+m_{V}^{\prime\left\{M_{\mathrm{WD}}\right\}}\left(t / f_{s}\right)-(m-M)_{V, \mathrm{PW} \text { VuldA } 8)}
\end{aligned}
$$

where $(m-M)_{V, \mathrm{PW} \mathrm{Vul}}=13.0$ is the distance modulus of PW Vul harboring a $0.83 M_{\odot}$ WD and we use Equation (A4), i.e.,

$$
\left[\frac{\dot{M}_{\mathrm{wind}}^{2}}{v_{\mathrm{ph}}^{2} R_{\mathrm{ph}}}\right]_{(t)}^{\left\{M_{\mathrm{wD}}\right\}}=\left[\frac{\dot{M}_{\mathrm{wind}}^{2}}{v_{\mathrm{ph}}^{2} R_{\mathrm{ph}}}\right]_{\left(t / f_{s}\right)}^{\left\{0.83 M_{\odot}\right\}}
$$

and Equation (A3), i.e.,

$$
m_{V}^{\prime\left\{M_{\mathrm{wD}}\right\}}\left(t / f_{s}\right)=-2.5 \log \left[\frac{\dot{M}_{\mathrm{wind}}^{2}}{v_{\mathrm{ph}}^{2} R_{\mathrm{ph}}}\right]_{(t)}^{\left\{M_{\mathrm{WD}}\right\}}+K_{V},
$$

to derive the last line of the above equation. The last line in Equation (A8) simply means that the model light curve 
$m_{V}^{\prime\left\{M_{\mathrm{WD}}\right\}}(t)$ in Figure 33 is shifted horizontally by $\log f_{s}$ and vertically by $2.5 \log f_{s}-(m-M)_{V, \mathrm{PW} \text { Vul }}$ to retrieve the absolute magnitude and real timescale. These retrieved absolute magnitudes are plotted in Figure 34 on the real timescale. We also tabulate the absolute magnitude, $M_{\mathrm{w}}$, at the end point of winds in Table 4 and plot them in Figure 35. These values of "CO nova 4" are in between those for "CO nova 2 " and "Ne nova 2." This confirms that our calibration of absolute magnitude is reasonable. Then, we retrieve the absolute magnitudes of all model light curves in Table 4 as

$$
M_{V}=m_{\mathrm{ff}}-\left(m_{\mathrm{w}}-M_{\mathrm{w}}\right)=m_{\mathrm{ff}}-\left(15.0-M_{\mathrm{w}}\right) .
$$

It should be noted that $K_{V}$ is a constant common to all WD masses (see Hachisu \& Kato 2010). Using Equations (A2) and (A3), we obtain $m_{\mathrm{ff}}-m_{V}^{\prime}=15.0-16.0=-1.0=$ $G^{\left\{0.83 M_{\odot}\right\}}-K_{V}$ at the bottom of the light curve (end of winds), where we directly read $m_{V}^{\prime}=m_{\mathrm{w}}=16.0$ for the $0.83 M_{\odot}$ model from Figure 8 . We recall that $G^{\left\{M_{\mathrm{WD}}\right\}}$ was defined to satisfy $m_{\mathrm{ff}}=15$ at the end point of optically thick winds in Equation (A2). We determine the value of $K_{V}$ from $K_{V}=$ $G^{\left\{0.83 M_{\odot}\right\}}+1.0$.

It is interesting to verify the consistency of our theoretical light curves with the empirical finding that the absolute magnitude 15 days after optical maximum, $M_{V}(15)$, is almost constant for novae. The value was first proposed by Buscombe \& de Vaucouleurs (1955) with $M_{V}(15)=-5.2 \pm$ 0.1 , followed by Cohen (1985) with $M_{V}(15)=-5.60 \pm 0.43$, van den Bergh \& Younger (1987) with $M_{V}(15)=-5.23 \pm$ 0.39, Capaccioli et al. (1989) with $M_{V}(15)=-5.69 \pm 0.42$, and Downes \& Duerbeck (2000) with $M_{V}(15)=-6.05 \pm$ 0.44 . The decline rates of our model light curves depend slightly on the chemical composition. We have already obtained $M_{V}(15)=-5.95 \pm 0.25$ for $0.55-1.2 M_{\odot}$ WDs with "CO nova 2 " and $M_{V}(15)=-5.6 \pm 0.3$ for $0.70-1.3 M_{\odot}$ WDs with "Ne nova 2" (Hachisu \& Kato 2010). This value is $M_{V}(15)=-5.4 \pm 0.4$ for $0.7-1.05 M_{\odot}$ WDs with "CO nova 4" as shown in Figure 34. In this figure, we plot, by magenta filled circles, the $V$ maxima of each model light curve corresponding to the $V$ maximum of PW Vul in Figure 33 and, by blue crosses, the absolute magnitudes of each model light curve 15 days after $V$ maximum, i.e., 15 days from each magenta filled circle. The obtained values are roughly consistent with the above empirical relations.

\section{A.4. MMRD relation}

The clear trend appearing from Figure 34 is that a more massive WD is systematically brighter at maximum (smaller $M_{V \text {,max }}$; see magenta filled circles near the mark B) and has a faster decline rate (smaller $t_{2}$ or $t_{3}$ time). The relation between $t_{3}$ (or $t_{2}$ ) and $M_{V, \max }$ for a nova is usually called "Maximum Magnitude vs. Rate of Decline" (MMRD) relation. Here $t_{3}\left(t_{2}\right)$ time is defined by 3-mag (2-mag) decay time from its maximum in units of day. Now we derive a theoretical MMRD relation for the "CO nova 4" chemical composition. Apparent maximum brightness $m_{V, \max }$ of each WD mass model light curve is expressed as $m_{V, \text { max }}=m_{V, \text { max }}^{\prime}+2.5 \log f_{\mathrm{s}}$ when the $t_{3}$ time is squeezed as $t_{3}=f_{\mathrm{s}} t_{3}^{\prime}$. Eliminating $f_{\mathrm{s}}$ from these two relations, we have $m_{V, \text { max }}=2.5 \log t_{3}+m_{V, \text { max }}^{\prime}-$ $2.5 \log t_{3}^{\prime}$. We obtained $t_{3}^{\prime}=80$ days and $m_{V \text { max }}^{\prime}=6.1$, where we measured $t_{3}^{\prime}$ and $m_{V \text {,max }}^{\prime}$ along our model light curves in Figure 33 Then, we obtain our MMRD relation as

$$
M_{V, \max }=m_{V, \max }-(m-M)_{V}
$$

$$
\begin{aligned}
& =2.5 \log t_{3}+m_{V, \max }^{\prime}-2.5 \log t_{3}^{\prime}-(m-M)_{V} \\
& =2.5 \log t_{3}-11.65
\end{aligned}
$$

where we use $(m-M)_{V}=13.0$ for PW Vul. Figure 36 shows this theoretical MMRD relation. This figure also shows the MMRD relation calibrated with V1668 Cyg (black dashed line taken from Hachisu \& Kato 2010). We also indicate the time-scaling factor $f_{\mathrm{s}}$ against the $\mathrm{PW}$ Vul light curves in the upper axis of the same figure.

For comparison, two empirical MMRD relations are plotted in the same figure, i.e., Kaler-Schmidt's law (blue solid line labeled "MMRD1": Schmidt 1957), i.e.,

$$
M_{V, \max }=-11.75+2.5 \log t_{3},
$$

and della Valle \& Livio's law (magenta solid line labeled "MMRD2": della Valle \& Livio 1995), i.e.,

$$
M_{V, \max }=-7.92-0.81 \arctan \left(\frac{1.32-\log t_{2}}{0.23}\right),
$$

where we use a relation of $t_{2} \approx 0.6 \times t_{3}$ for the optical light curves that follow the universal decline law (Hachisu \& Kato 2006).

Figure 36 also shows observational points for individual novae (red filled circles), taken from Table 5 of Downes \& Duerbeck (2000). Note that we remeasured the $t_{3}$ time of PW Vul along our model light curve, which resulted in $t_{3}=80$ days and $M_{V, \max }=-6.9$ as shown by a large black open circle. This data point is leftside to Downes \& Duerbeck's (2000) estimate $\left(t_{3}=126\right.$ day and $\left.M_{V, \max }=-6.7\right)$ denoted by a large red filled circle.

The scatter of the observed data with respect to the MMRD formulae in Figure 36 is generally large, and larger than the observational errors. This strongly suggests that the scatter is due to the presence of a second parameter. Hachisu \& Kato (2010) pointed out that the main parameter governing the MMRD relation is the WD mass (represented by the timescaling factor $f_{\mathrm{s}}$ in Figure 36, the second parameter being the initial envelope mass (or the mass accretion rate of the WD). In turn, the initial envelope mass (ignition mass) depends on the mass accretion rate to the WD (see, e.g., Figure 3 of Kato et al. 2014) such that the lower the mass accretion rate is, the larger the envelope mass is. This simply means that, for the same WD mass, novae are brighter/fainter for lower/higher mass accretion rates. Hachisu \& Kato (2010) clearly showed the dependence of maximum brightness on the initial envelope mass (see their Figures 8 and 15). We further show fainter examples of maximum brightness, RS Oph and M31N2008-12a, both of which are recurrent novae with $\sim 20$ and $\sim 1$ yr recurrence periods, respectively. We conclude that this second parameter, the initial envelope mass, can reasonably explain the scatter of individual novae from the empirical MMRD relations so far proposed (see Figure 15 of Hachisu \& Kato 2010).

\section{B. TIME-STRETCHING METHOD}

The distance modulus of PW Vul can be estimated, in a very different way, from a resemblance between PW Vul and other optically well-observed novae. Hachisu \& Kato (2006) found that nova light curves follow a universal decline law when free-free emission dominates the spectrum in optical and NIR regions. Using this property, Hachisu \& Kato (2010) found that, if two nova light curves overlap each other after one of the two is squeezed/stretched by a factor of $f_{s}$ 
$\left(t^{\prime}=t / f_{s}\right)$ in the time direction, the brightnesses of the two novae obey the relation of

$$
m_{V}^{\prime}=m_{V}-2.5 \log f_{s},
$$

which is the same as Equation $\mathrm{A6}$. Using this property with calibrated nova light curves, we can estimate the absolute magnitude of a target nova. Figure 2 shows timenormalized light curves of PW Vul, V1668 Cyg, V1974 Cyg, and V533 Her against that of V1500 Cyg, similar to Figure 41 of Hachisu \& Kato (2014), but we used the reanalyzed data. The $V$ light curve, $B-V$ and $U-B$ color curves of PW Vul are well squeezed to match the other ones. Note that the dereddened $(B-V)_{0}$ and $(U-B)_{0}$ of each nova also follow a general course in the color-color diagram (i.e., overlap each other; see Hachisu \& Kato 2014, for the general course of $U B V$ color evolution of novae). These five novae obey the relations of

$$
\begin{aligned}
(m-M)_{V, \mathrm{~V} 1500 \mathrm{Cyg}} & =12.3 \\
& =(m-M)_{V, \mathrm{PW} \mathrm{Vul}}+\Delta V-2.5 \log 0.182 \\
& \approx 13.0-2.6+1.85=12.25 \\
& =(m-M)_{V, \mathrm{~V} 1668 \mathrm{Cyg}}+\Delta V-2.5 \log 0.44 \\
& \approx 14.25-2.9+0.90=12.25 \\
& =(m-M)_{V, \mathrm{~V} 1974 \mathrm{Cyg}}+\Delta V-2.5 \log 0.42 \\
& \approx 12.2-0.8+0.95=12.35 \\
& =(m-M)_{V, \mathrm{~V} 533 \mathrm{Her}}+\Delta V-2.5 \log 0.54 \\
& \approx 10.8+0.8+0.67=12.27,
\end{aligned}
$$

where $\Delta V$ is the difference of apparent brightness obtained in Figure 2 by which the $V$ light curve of each nova is shifted up or down against that of V1500 Cyg. The time-scaling factors are also obtained in this figure as $f_{s}=0.182$ for PW Vul, $f_{s}=0.44$ for V1668 Cyg, $f_{s}=0.42$ for V1974 Cyg, and $f_{s}=0.54$ for V533 Her, each against that of V1500 Cyg. We obtained these stretching factors by shifting horizontally each light curve to overlap them. The apparent distance moduli of V1500 Cyg, V1668 Cyg, and V1974 Cyg were calibrated as $(m-M)_{V, \mathrm{~V} 1500 \mathrm{Cyg}}=12.3,(m-M)_{V . \mathrm{V} 1668 \mathrm{Cyg}}=14.25$, and $(m-M)_{V, \mathrm{~V} 1974 \text { Cyg }}=12.2$ in Hachisu \& Kato (2014). These three are all consistent with each other. The distance modu-

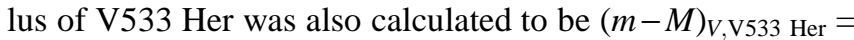
10.8. Hachisu \& Kato (2014) obtained the distance-modulus of PW Vul based on this time-stretching method as $(m-M)_{V}=$ $13.0 \pm 0.1$, which is consistent with Equation (1). This is a strong support to our adopted values of $E(B-V)=0.55$ and $d=1.8 \mathrm{kpc}$.

\section{REFERENCES}

Aller, L. H. 1984, Physics of Thermal Gaseous Nebulae (Dortlechit: D. Reidel).

Andreae, J., \& Drechsel, H. 1990, Physics of Classical Novae, eds. A. Cassatella and R. Viotti (Berin: Springer-Verlag), 204

Andreae, J., Drechsel, H., Snijders, M. A. J., \& Cassatella, A. 1991, A\&A, 244,111

Andreä, J., Drechsel, H., \& Starrfield, S. 1994, A\&A, 291, 869

Arenas, J., Catalán, M. S., Augusteijn, T., \& Retter, A. 2000, MNRAS, 311, 135

Arkhipova, V. P., \& Zaitseva, G. V. 1976, SvAL, 2, 35

Arkhipova, V. P., Burlak, M. A., \& Esipov, V. F. 2000, Astronomy Letters, 26,372

Barnes, T. G., \& Evans, N. R. 1970, PASP, 82, 889

Barry, R. K., Mukai, K., Sokoloski, J. L., et al. 2006, RS Ophiuchi (2006) and the Recurrent Nova Phenomenon, ASP Conference Series, 401, 52

Budding, E. 1983, IAU Circ., 3853, 2

Buscombe, W., \& de Vaucouleurs, G. 1955, The Observatory, 75, 170

Campbell, L., \& Shapley, H. 1923, Annals of the Astronomical Observatory of Harvard College, 81, 113

Candy, M. P., Alcock, G. E. D., \& Zissell, R. E. 1967, IAU Circ., 2022, 1

Capaccioli, M., della Valle, M., Rosino, L., D’Onofrio, M. 1989, AJ, 97, 1622
Cassatella, A., Altamore, A., \& González-Riestra, R. 2002, A\&A, 384, 1023

Child, L. 1901, MNRAS, 61, 483

Chincarini, G. 1964, PASP, 76, 289

Chochol, D., \& Pribulla, T. 1997, CoSka, 27, 53

Chochol, D., \& Pribulla, T. 1998, CoSka, 28, 121

Cohen, J. G. 1985, ApJ, 292, 90

Cohen, J. G., \& Rosenthal, A. J. 1983, ApJ, 268, 689

Dawson, B. H. 1926, AJ, 36, 148

de Freitas Pacheco, J. A., \& Codina, S. J. 1985, MNRAS, 214, 481

della Valle, M. 1991, A\&A, 252, L9

della Valle, M., \& Livio, M. 1995, ApJ, 452, 704

Diaz, M. P. \& Steiner, J. E. 1989, ApJ, 339, L41

Diaz, M. P., \& Steiner, J. E. 1994, ApJ, 425,252

Downes, R. A., \& Duerbeck, H. W. 2000, AJ, 120, 2007

Drechsel, H., Rahe, J., Duerbeck, H. W., Kohoutek, L., \& Seitter, W. C. 1977, A\&AS, 30, 323

Duerbeck, H. W. 1981, PASP, 93, 165

Duerbeck, H. W., Geffert, M., Nelles, B., Dummler, R., \& Nolte, M. 1984, IBVS, 2641, 1

Ennis, D., Becklin, E. E., Beckwith, S., et al. 1977, ApJ, 214, 478

Evans, A., Callus, C. M., Whitlock, P. A., \& Laney, D. 1990, MNRAS, 246, 527

Evans, A., Gehrz, R. D., Geballe, T. R., et al. 2003, AJ, 126, 1981

Friedjung, M. 1992, A\&A, 262, 487

Friedjung, M., Dennefeld, M., \& Voloshina, I. 2010, A\&A, 521, A84

Gallagher, J. S., \& Holm, A. V. 1974, ApJL, 189, L123

Gallagher, J. S., \& Ney, E. P. 1976, ApJL, 204, L35

Gaposchkin, S. 1956, AJ, 61, 36

Gehrz, R. D., Harrison, T. E., Ney, E. P., et al. 1988, ApJ, 329, 894

Gehrz, R. D., Truran, J. W., Williams, R. E., \& Starrfield, S. 1998, PASP 110,3

Goranskij, V. P., Shugarov, S. Y., Katysheva, N. A., et al. 2000, IBVS, 4852

Goranskij, V. P., Katysheva, N. A., Kusakin, A. V., et al. 2007, Astrophysical Bulletin, 62, 125

Gore, J. E. 1901, MNRAS, 62, 156

González-Riestra, R., Shore, S. N., Starrfield, S., \& Krautter, J. 1996, IAU Circ., 6295, 1

Grevesse, N., \& Anders, E. 1989, Cosmic Abundances of Matter, ed. C. J.

Waddington (New York: AIP), 1

Grygar, J. 1969, IBVS, 371, 1

Hachisu, I., \& Kato, M. 2001, ApJ, 558, 323

Hachisu, I., \& Kato, M. 2004, ApJL, 612, L57

Hachisu, I., \& Kato, M. 2006, ApJS, 167, 59

Hachisu, I., \& Kato, M. 2007, ApJ, 662, 552

Hachisu, I., \& Kato, M. 2010, ApJ, 709, 680

Hachisu, I., \& Kato, M. 2014, ApJ, 785, 97

Hachisu, I., Kato, M., \& Cassatella, A. 2008, ApJ, 687, 1236

Hachisu, I., Kato, M., Kiyota, S., et al. 2006, ApJL, 651, L141

Hachisu, I., Kato, M., \& Luna, G. J. M. 2007, ApJ, 659, L153

Harrison, T. E., Bornak, J., McArthur, B. E., \& Benedict, G. F. 2013, ApJ, 767,7

Harman, D. J., \& O’Brien, T. J. 2003, MNRAS, 344, 1219

Hassall, B. J. M., Snijders, M. A. J., Harris, A. W., et al. 1990, in Physics of Classical Novae, ed. A. Cassatella \& R. Viotti (Berin: Springer), 202

Hauschildt, P. H., Starrfield, S., Shore, S. N., et al. 1994, AJ, 108, 1008

Hauschildt, P. H., Starrfield, S., Shore, S. N., Allard, F., \& Baron, E. 1995 , ApJ, 447, 829

Hauschildt, P. H., Shore, S. N., Schwarz, G. J., et al., ApJ, 490, 803

Horne, K., Welsh, W., \& Wade, R. A. 1993, ApJ, 410, 357

Hric, L., Petrík, K., Urban, Z., \& Hanžl, D. 1998, A\&AS, 133, 211

Hutchings, J. B. 1970, Publications of the Dominion Astrophysical Observatory, 13,347

lijima, T. 2006, A\&A, 451, 563

IIjima, T. 2007a, CBET, 934, 1

Iijima, T. 2007b, CBET, 1006,

Iijima, T., Rosino, L., \& della Valle, M. 1998, A\&A, 338, 1006

Kamath, U. S., \& Ashok, N. M. 1999, A\&AS, 136, 107

Kato, M., \& Hachisu, I., 1994, ApJ, 437, 802

Kato, M., \& Hachisu, I., 2009, ApJ, 699, 1293

Kato, M., \& Hachisu, I., 2011, ApJ, 743, 157

Kato, M., Hachisu, I., \& Cassatella, A. 2009, ApJ, 704, 1676

Kato, M., Hachisu, I., Cassatella, A., \& González-Riestra, R. 2011, ApJ, 727,72

Kato, M., Mikołajewska, J., \& Hachisu, I. 2012, ApJ, 750, 5

Kato, M., Saio, H., Hachisu, I., \& Nomoto, K. 2014, ApJ, 793, 136

Kawara, K., Maihara, T., Noguchi, K., et al. 1976, PASJ, 28, 163

Kiss, L. \& Sarneczky, K. 2007, CBET, 1039, 1

Kohoutek, L. 1981, MNRAS, 196, 87P

Kolotilov, E. A., Munari, U., \& Yudin, B. F. 1995, MNRAS, 275, 185

Kosai, H., Takana, W., Watanabe, T., et al. 1984, IAU Circ., 3963, 2

Krautter, J., Beuermann, K., Leitherer, C., et al. 1984, A\&A, 137, 307

Kürster, M., \& Barwig, H. 1988, A\&A, 199, 201

Lockwood, G. W., \& Millis, R. L. 1976, PASP, 88, 235

Lyke, J. E., \& Campbell, R. D. 2009, AJ, 138, 1090

Malakpur, I. 1975, Ap\&SS, 38, 403

Mannery, E. J. 1970, PASP, 82, 626 
Marshall, D. J., Robin, A. C., Reylé, C., Schultheis, M., \& Picaud, S. 2006, A\&A, 453, 635

Miroshnichenko, A. S. 1988, SvA, 32, 298

Mollerus, B. 1969, A\&A, 3, 376

Morales-Rueda, L., Still, M. D., Roche, P., Wood, J. H., Lockley, J. J. 2002, MNRAS, 329, 597

Morisset, C., \& Péquignot, D. 1996, A\&A, 312, 135

Munari, U., Goranskij, V. P., Popova, A. A., et al. 1996, A\&A, 315, 166

Munari, U., Orio, M., Valentini, M., et al. 2007, CBET, 1010, 1

Munari, U., Yudin, B. F., Kolotilov, E. A., et al. 1994b, A\&A, 284, L9

Nha, Il-Seong 1967, IBVS, 238, 1

Naito, H., Matsuda, K., \& Yamaoka, H. 2007, CBET, 934, 2

Nakano, S., Kanatsu, K., Kawanishi, K., et al. 1993, IAU Circ., 5902, 1

Nakano, S., Sakurai, Y., Itagaki, K., \& Koff, R. 2007, IAU Circ., 8832, 1

Ness, J.-U., Schwarz, G., Starrfield, S., et al. 2008, AJ, 135, 1328

Nishimaki, Y., Yamamuro, T., Motohara, K., Miyata, T., \& Tanaka, M. 2008, PASJ, 60, 191

Nomoto, K. 1982, ApJ, 253, 798

O’Connell, D. J. K. 1968, IBVS, 313,

Öegelman, H., Krautter, J., \& Beuermann, K. 1987, A\&A, 177, 110

Onderlička, B., \& Vetešník, M. 1968, BAICz, 19, 99

Orio, M., Covington, J., \& Ögelman, H. 2001, A\&A, 373, 542

Payne-Gaposchkin, C. 1957, The Galactic Novae (Amsterdam: North-Holland)

Péquignot, D., Petitjean, P., Boisson, C., \& Krautter, J. 1993, A\&A, 271, 219

Petitjean, P., Boisson, C., \& Pequignot, D. 1990, A\&A, 240, 433

Pfau, W. 1976, IBVS, 1106, 1

Poggiani, R. 2008, NewA, 13, 557

Poggiani, R. 2010, NewA, 15, 657

Poggiani, R. 2012, Memorie della Societa Astronomica Italiana, 83, 753

Prialnik, D., \& Kovetz, A. 1995, ApJ, 445, 789

Rafanelli, P., \& Rosino, L. 1978, A\&AS, 31, 337

Rambaut, A. A. 1901a, MNRAS, 61, 348

Rambaut, A. A. 1901b, MNRAS, 61, 390

Rambaut, A. A. 1901c, MNRAS, 61, 467

Rambaut, A. A. 1901d, MNRAS, 61, 544

Rambaut, A. A. 1901e, MNRAS, 62, 78

Rambaut, A. A. 1902, MNRAS, 62, 586

Rambaut, A. A. 1903, MNRAS, 63, 509

Rieke, G. H., \& Lebofsky, M. J. 1985, ApJ, 288, 618

Robb, R. M., \& Scarfe, C. D. 1995, MNRAS, 273, 347

Robinson, L. J., \& Ashbrook, J. 1968, IBVS, 252, 1

Rosino, L., \& Iijima, T. 1987, Ap\&SS, 130, 157

Rudy, R. J., Lynch, D. K., Russell, R. W., \& Woodward, C. E. 2007b, IAU Circ., 8884, 2

Rudy, R. J., Venturini, C. C., Lynch, D. K., Mazuk, S., \& Puetter, R. C. 2002, ApJ, 573, 794

Saizar, P., Starrfield, S., Ferland, G. J., et al. 1991, ApJ, 367, 310

Sanyal, A. 1974, ApJS, 28, 115
Schlafly, E. F., \& Finkbeiner, D. P. 2011, ApJ, 737, 103

Schmidt, G. D., Smith, P., \& Elston, R. 1987, IAU Circ., 4415, 2

Schmidt, G. D., \& Stockman, H. S. 1987, IAU Circ., 4458, 1

Schmidt, Th. 1957, ZA, 41, 182

Schwarz, G. J., Starrfield, S., Shore, S. N., \& Hauschildt, P. H. 1997, MNRAS, 290, 75

Schwarz, G. J., Ness, J.-U., Osborne, J. P., et al. 2011, ApJS, 197, 31

Seaton, M. J. 1979, MNRAS, 187, 73P

Shanley, L., Ögelman, H., Gallagher, J. S., Orio, M., \& Krautter, J. 1995, ApJL, 438, L95

Sharp, M. C. 1901, MNRAS, 61, 398

Shen, L.-Z., et al. 1964, AcASn, 12, 83

Shore, S. N., Starrfield, S., Gonzalez-Riestrat, R., Hauschildt, P. H., \& Sonneborn, G. 1994, Natur, 369, 539

Slavin, A. J., O'Brien, T. J., \& Dunlop, J. S. 1994, MNRAS, 266, L55

Slavin, A. J., O'Brien, T. J., \& Dunlop, J. S. 1995, MNRAS, 276, 353

Solf, J. 1983, ApJ, 273, 647

Spencer Jones, H. 1925, The Observatory, 48, 261

Spencer Jones, H. 1931, Annals of the Cape Observatory, 10, 9.1

Stokes, A. J. 1967, IBVS, 224, 1

Strope, R., Schaefer, B. E., \& Henden, A. A. 2010, AJ, 140, 34

Tanaka, J., Nogami, D., Fujii, M., et al. 2011, PASJ, 63, 911

Tang, S., Bildsten, L., Wolf, W. M., et al. 2014, ApJ, 786, 61

Tempesti, P. 1979, AN, 300, 51

Terzan, A. 1968, JO, 51, 329

Tylenda, R. 1978, Acta Astron., 28, 333

van den Bergh, S., \& Younger, P. F. 1987, A\&AS, 70, 125

van Genderen, A. M. 1963, BAN, 17, 293

Verbunt, F. 1987, A\&AS, 71, 339

Warner, B. 1995, Cataclysmic Variable Stars, (Cambridge: Cambridge Univ. Press)

Whitelock, P. A., Carter, B. S., Feast, M. W., et al. 1984, MNRAS, 211, 421

Williams, A. S. 1901a, MNRAS, 61, 337

Williams, A. S. 1901b, MNRAS, 61, 396

Williams, A. S. 1901b, MNRAS, 61, 480

Williams, A. S. 1901d, MNRAS, 61, 550

Williams, A. S. 1902, MNRAS, 62, 589

Williams, A. S. 1919, MNRAS, 79, 362

Williams, R. E., \& Gallagher, J. S. 1979, ApJ, 228, 482

Williams, R. E., Hamuy, M., Phillips, M. M., et al. 1991, ApJ, 376, 72

Williams, P. M., Longmore, A. J., \& Geballe, T. R. 1996, MNRAS, 279, 804 Williams, R. E., Woolf, N. J., Hege, E. K., Moore, R. L., \& Kopriva, D. A. 1978, ApJ, 224, 171

Wright, A. E., \& Barlow, M. J. 1975, MNRAS, 70, 41

Wu, C.-C., Holm, A. V., Panek, R. J., et al. 1989, ApJ, 339, 443

Yamaoka, H., Haseda, K., \& Nakamura, Y. 2007, IAU Circ., 8832, 2 